\title{
Pentaquark baryons in SU(3) quark model
}

\author{
Yongseok $\mathrm{Oh}^{*}$ \\ Department of Physics and Astronomy, University of Georgia, Athens, GA 30602, U.S.A. \\ Hungchong $\operatorname{Kim}^{\dagger}$ \\ Institute of Physics and Applied Physics, Yonsei University, Seoul 120-749, Korea
}

(Dated: February 1, 2008)

\begin{abstract}
We study the SU(3) group structure of pentaquark baryons which are made of four quarks and one antiquark. The pentaquark baryons form $\mathbf{1}, \mathbf{8}, \mathbf{1 0}, \overline{\mathbf{1 0}}, \mathbf{2 7}$, and $\mathbf{3 5}$ multiplets in SU(3) quark model. First, the flavor wave functions of all the pentaquark baryons are constructed in SU(3) quark model and then the flavor $\mathrm{SU}(3)$ symmetry relations for the interactions of the pentaquarks with three-quark baryons and pentaquark baryons are obtained. Constructing the general interactions in $\mathrm{SU}(3)$ could be important for understanding the pentaquark baryon properties from reaction mechanisms. We also discuss possible pentaquarks in 27-plet and 35-plet and their decay channels that can be used to identify them in future experiments. The mass sum rules for the pentaquark baryons are also presented.
\end{abstract}

PACS numbers: 12.39.-x, 14.20.Jn

\section{INTRODUCTION}

The discovery of $\Theta^{+}(1540)$ by the LEPS Collaboration at SPring-8 [1] and the observations of the subsequent experiments [2-13] have initiated great interests in exotic baryons in hadron physics. Because of its positive strangeness, the minimal quark content must be $u d u d \bar{s}$ and hence $\Theta^{+}$is an exotic pentaquark state. The observation of $\Xi^{--}(1862)$ by NA49 Collaboration [14] may suggest that $\Xi(1862)$ forms pentaquark antidecuplet with $\Theta^{+}(1540)$, which must be confirmed by other experiments [15]. ${ }^{1}$ Recently, a pentaquark state containing anti-charm quark was observed by H1 Collaboration [18]. Because of the observation of pentaquark states in various reaction channels, the existence of pentaquark baryons now becomes widely accepted. ${ }^{2}$ Thus it is natural to search for the other pentaquark baryons which have been predicted by hadron models.

Pentaquark baryons may be pure exotic or crypto-exotic. The pure exotic states can easily be identified by their unique quantum numbers, but the crypto-exotic states are hard to be identified as their quantum numbers can also be generated by three-quark states. Therefore, it is crucial to have careful analyses for their decay channels. Historically, there have been many efforts to find pentaquark states with the development of quark models, which, however, failed to observe $\Theta(1540)$. The efforts to search for pentaquark baryons until 1980's were summarized in Refs. [20, 21]. (See also Ref. [22]. $)^{3}$ Early theoretical works on exotic baryons can be found, e.g., in Refs. [43]. Rigorous theoretical studies were then performed for heavy quark sector, i.e., pentaquark baryons with one anti-charmed quark or anti-bottom quark. In the pioneering work of Lipkin [23] and Grenoble group [24], the anti-charmed pentaquark with one strange quark was shown to have the same binding energy as the $H$ dibaryon in the heavy quark mass limit and in the $\mathrm{SU}(3)$ limit. Then it has been studied in more sophisticated quark models [25, 26] and in Skyrme model [27, 28]. Following the first experimental search for heavy pentaquarks [29], the observation of $\Theta^{+}(1540)$ and $\Theta_{c}(3099)$ has brought new interests in this subject [30-35].

\footnotetext{
*Electronic address: yoh@physast.uga.edu

${ }^{\dagger}$ Electronic address: hung@phya.yonsei.ac.kr

1 The recent report from WA89 Collaboration $[16,17]$ shows no evidence for $\Xi(1860)$ in $\Sigma^{-}$-nucleus collisions.

${ }^{2}$ However, higher statistics experiments are required to firmly establish the observed resonances. See Ref. [17] for a compilation of positive and negative reports on the existence of $\Theta^{+}(1540)$ in various experiments. Another interpretation for the $\Theta^{+}(1540)$ peak was suggested by Ref. [19].

${ }^{3}$ In the literature we could find several resonances that were claimed to be crypto-exotic states. For example, $X(1340), X(1450)$, and $X(1640)$ were reported by Ref. [36] and $X(3520)$ by Ref. [37]. $X(1390), X(1480)$, and $X(1620)$ that have isospin $I \geq 5 / 2$ were observed by Ref. [38], and Ref. [39] reported $\Sigma(3170)$. Most of them were found to have narrow widths, but their existence was not confirmed and questioned by later experiments $[40,41]$. SPHINX Collaboration has reported the existence of $X(2000), X(2050)$, and $X(2400)$ that are expected to have the quark content of $u u d s \bar{s}[42]$, whose existence should be carefully re-examined by other experiments.
} 
In the light quark sector, pentaquark states were anticipated in the Skyrme model $[44,45]$. The first detailed study on antidecuplet was made by Diakonov et al. [46, 47], which predicted a very narrow $\Theta^{+}$with a mass around 1530 $\mathrm{MeV}$ by identifying $N(1710)$ as the nucleon analogue of the antidecuplet. After the discovery of $\Theta^{+}(1540)$ there have been lots of theoretical models and ideas to explain the structure of pentaquark baryons and to search for the other pentaquark states. The subsequent theoretical studies include the soliton models [48-52], QCD sum rules [53], large $N_{c}$ QCD [54,55], and lattice calculation [56-58], etc. As the quark models have provided a cornerstone for hadron physics, it is legitimate to start with the quark models and study the structure of pentaquark baryons. In Ref. [59], Karliner and Lipkin suggested a triquark-diquark model, where, for example, $\Theta^{+}$is a system of $(u d)-(u d \bar{s})$. In Ref. [60], Jaffe and Wilczek advocated a diquark-diquark-antiquark model so that $\Theta^{+}$is $(u d)-(u d)-\bar{s}$. In this model, they also considered the mixing of the pentaquark antidecuplet with the pentaquark octet, which makes it different from the SU(3) soliton models where the octet describes the normal (three-quark) baryon octet. Assuming that the nucleon and $\Sigma$ analogues are in the ideal mixing of the octet and antidecuplet, the nucleon analogue is then identified as the Roper resonance $N(1440)$. In Ref. [61], it was pointed out that the Roper resonance $N(1710)$ should be excluded as a pure antidecuplet state. This is because, within $\mathrm{SU}(3)$ symmetry, antidecuplet does not couple to decuplet and meson octet, whereas $N(1710)$ has a large branching ratio into $\pi \Delta$ channel. Therefore, mixing with other multiplets is required if one wants to identify $N(1710)$ as a pentaquark crypto-exotic state. However, recent study for the ideal mixing between antidecuplet and octet states shows that the ideally mixed state still has vanishing coupling with the $\pi \Delta$ channel [62,63], which excludes $N(1440)$ as a pentaquark state. This shows the importance of reaction/decay studies in identifying especially crypto-exotic pentaquark states. More discussions on the quark model predictions based on the diquark picture can be found, e.g., in Refs. [61, 64]. Predictions on the antidecuplet spectrum in various quark models can be found, e.g., in Refs. [65-70]

In quark model, pentaquark baryons form six multiplets, $\mathbf{1}, \mathbf{8}, \mathbf{1 0}, \overline{\mathbf{1 0}}, \mathbf{2 7}$, and $\mathbf{3 5}$. The other type resonances are thus expected together with antidecuplet, particularly the isovector $\Theta$ belonging to 27-plet and isotensor $\Theta$ as a member of 35-plet. The interest in this direction has been growing [65, 71-74] and it is important to know the interactions and decay channels to search for the other pentaquark baryons.

Furthermore, understanding the $\Theta^{+}$properties such as spin-parity requires careful analyses of production mechanisms including $\gamma N \rightarrow \bar{K} \Theta$ [75-80], $\gamma N \rightarrow \bar{K}^{*} \Theta$ [81], $\gamma N \rightarrow K \pi \Theta$ [82] $\gamma N \rightarrow K^{+} K^{-} \Theta$ [83], $N N \rightarrow Y \Theta$ [84-86], and $K N \rightarrow K \pi N$ [87]. Most model predictions for those production processes, however, do not consider the intermediate pentaquark baryons in its production mechanisms as the unknown inputs like the electromagnetic and strong couplings of pentaquark baryons are required. Therefore, knowing the interaction Lagrangian of pentaquark baryons are necessary.

The physical pentaquark states would be mixtures of various multiplets as in the chiral soliton model [73]. Such a representation mixing is induced by $\mathrm{SU}(3)$ symmetry breaking and it can be studied in quark potential models. Therefore, it is desirable to obtain the full set of pentaquark wave functions in quark model for further investigation. In this paper, we construct the flavor wave functions of pentaquark baryons in $\mathrm{SU}(3)$ quark model. There are several works in this direction and the flavor wave functions of antidecuplet has been obtained in Refs. [62, 67, 68, 88]. (See also Ref. [55] for the relation between the wave functions of pentaquark baryons in quark model and Skyrme model in the large $N_{c}$ limit.) The SU(3) symmetric interactions for antidecuplet have been studied in Refs. [61-63], which motivated the development of a chiral Lagrangian for antidecuplet $[89,90]$. In this work, we extend the SU(3) quark model to pentaquark states and obtain the flavor wave functions of all pentaquark states including singlet, octet, decuplet, antidecuplet, 27-plet, and 35-plet. Then we obtain the SU(3) symmetric Lagrangian of pentaquark-threequark and pentaquark-pentaquark interactions with meson octet.

This paper is organized as follows. In the next Section, we start with the quark and antiquark operators and form a diquark state. Then by taking direct product of two diquarks and one antiquark, we form the pentaquark states in tensor notation. The physical baryon states are, therefore, represented by SU(3) tensors as shown in Sect. III. This allows us to obtain the flavor wave functions of pentaquark baryons by identifying each $\mathrm{SU}(3)$ tensor with physical pentaquark states. Although the procedure in this paper follows the diquark-diquark-antiquark picture, the obtained wave function is general as we do not impose any dynamics to the quarks. In Sect. IV, we develop SU(3) symmetric Lagrangian for pentaquark baryons. We consider the interactions of pentaquarks with normal three-quark baryons (octet and decuplet) and meson octet. Then it is extended to construct the interactions of pentaquark baryons with (other) pentaquark multiplets and meson octet. The $\mathrm{SU}(3)$ symmetric case is considered in this work, but the symmetry breaking can be included in a standard way [91]. In Sect. V, the mass relations among pentaquark baryons are obtained. We found Gell-Mann-Okubo mass relation for 27-plet and equal spacing rules not only for $\mathbf{1 0}$ and $\overline{\mathbf{1 0}}$ but also for $\mathbf{2 7}$ and $\mathbf{3 5}$. Section VI contains a summary. 


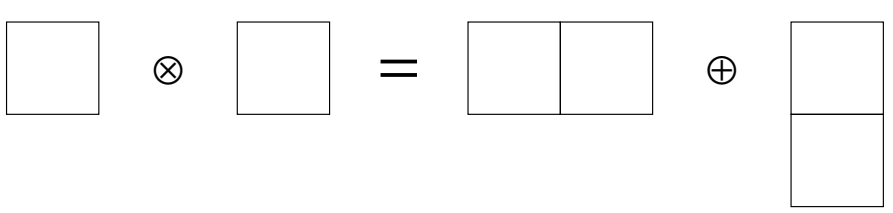

FIG. 1: Product of two quarks, $\mathbf{3} \otimes \mathbf{3}=\mathbf{6} \oplus \overline{\mathbf{3}}$.

\begin{tabular}{c|c|c}
\hline \hline irreducible tensor & type & notation \\
\hline$S_{j k}$ & $(2,0)$ & $\mathbf{6}$ \\
$T^{i}$ & $(0,1)$ & $\overline{\mathbf{3}}$ \\
\hline \hline
\end{tabular}

TABLE I: Irreducible tensors of a diquark.

\section{WAVE FUNCTIONS OF PENTAQUARK BARYONS}

We start with the representations for quark and antiquark. We denote a quark by $q_{i}$ and an antiquark by $q^{i}$ with $i=1,2,3$, so that $q_{1}, q_{2}$, and $q_{3}$ are $u, d$, and $s$ quark, respectively. The inner products of the quark and antiquark operators are normalized as

$$
\left(q_{i}, q_{j}\right)=\delta_{i j}, \quad\left(q^{i}, q^{j}\right)=\delta^{i j}, \quad\left(q_{i}, q^{j}\right)=0 .
$$

\section{A. Diquark}

We first construct a diquark by a direct product of two quarks. In flavor SU(3) group, the quarks $q_{i}$ are in the fundamental representation 3, which is described by a box in Young tableau. The direct product of two quarks then gives $\mathbf{3} \otimes \mathbf{3}=\mathbf{6} \oplus \overline{\mathbf{3}}$ as shown in Fig. 1 . In $(p, q)$ notation, $\mathbf{6}$ is $(2,0)$ type and $\overline{\mathbf{3}}$ is $(0,1)$ type. Generally, $(p, q)$ type can be represented by a tensor $T_{a_{1}, \ldots, a_{p}}^{b_{1}, \ldots, b_{q}}$, which is completely symmetric in upper indices and in lower indices, namely,

$$
T_{a_{1}, a_{2}, \ldots, a_{p}}^{b_{1}, b_{2}, \ldots, b_{q}}=T_{a_{2}, a_{1}, \ldots, a_{p}}^{b_{1}, b_{2}, \ldots, b_{q}}=T_{a_{1}, a_{2}, \ldots, a_{p}}^{b_{2}, b_{1}, \ldots, b_{q}}=T_{a_{2}, a_{1}, \ldots, a_{p}}^{b_{2}, b_{1}, \ldots, b_{q}},
$$

etc. It is also traceless on every pair of indices so that

$$
T_{a_{1}, a_{2}, \ldots, a_{p}}^{a_{1}, b_{2}, \ldots, b_{q}}=0 .
$$

Therefore, $\mathbf{6}$ is represented by a tensor $T_{i j}$ and $\overline{\mathbf{3}}$ is by $T^{i}$ like in the case of antiquarks.

Explicitly, by symmetrizing and anti-symmetrizing, the product of two quarks is written as

$$
q_{j} q_{k}=\frac{1}{\sqrt{2}} S_{j k}+\frac{1}{2 \sqrt{2}} \epsilon_{i j k} T^{i}
$$

where

$$
\left(\begin{array}{c}
S_{j k} \\
A_{j k}
\end{array}\right)=\frac{1}{\sqrt{2}}\left(q_{j} q_{k} \pm q_{k} q_{j}\right)
$$

and

$$
T^{i}=\epsilon^{i j k} A_{j k},
$$

so that $S_{j k}$ and $T^{i}$ represent $\mathbf{6}$ and $\overline{\mathbf{3}}$, respectively. The inner products of $S_{j k}$ and $T^{i}$ are then obtained as

$$
\left(S_{j k}, S_{l m}\right)=\delta_{j l} \delta_{k m}+\delta_{j m} \delta_{k l}, \quad\left(T^{i}, T^{j}\right)=4 \delta^{i j} .
$$

Since $S_{j k}$ and $T^{i}$ are irreducible representations, the inner product $\left(S_{j k}, T^{i}\right)$ vanishes. 
(a)

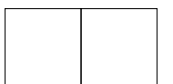

$\otimes$

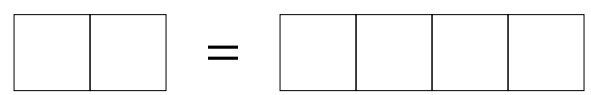

$\oplus$

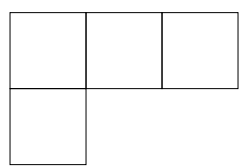

$\oplus$

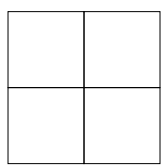

(b)

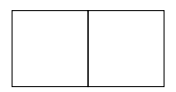

$\otimes$

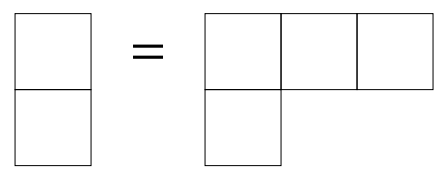

$\oplus$

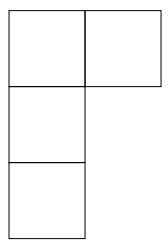

(c)

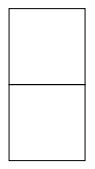

$\otimes$

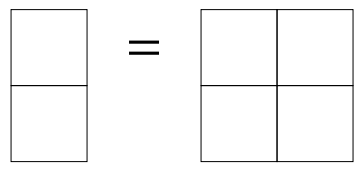

$\oplus$

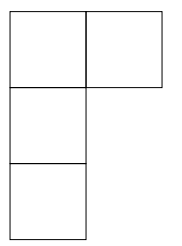

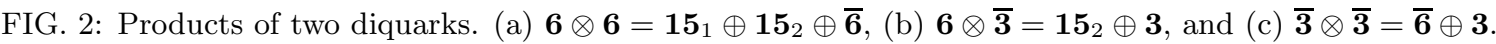

\section{B. Two diquarks}

Since one diquark is either in $\mathbf{6}$ or $\overline{\mathbf{3}}$, the direct product of two diquarks contains $\mathbf{6} \otimes \mathbf{6}, \mathbf{6} \otimes \overline{\mathbf{3}}, \overline{\mathbf{3}} \otimes \mathbf{6}$, and $\overline{\mathbf{3}} \otimes \overline{\mathbf{3}}$ :

$$
\left(q_{j} q_{k}\right)\left(q_{l} q_{m}\right)=\frac{1}{2} S_{j k} S_{l m}+\frac{1}{4}\left(\epsilon_{i j k} T^{i} S_{l m}+\epsilon_{i l m} S_{j k} T^{i}\right)+\frac{1}{8} \epsilon_{i j k} \epsilon_{n l m} T^{i} T^{n} .
$$

The direct products of each multiplets are shown in Fig. 2.

We start with noting that the product $\overline{\mathbf{3}} \otimes \overline{\mathbf{3}}$ is decomposed into two parts as

$$
T^{i} T^{j}=2 \sqrt{2} S^{i j}+2 \epsilon^{i j k} T_{k}
$$

where

$$
\begin{aligned}
S^{i j} & =\frac{1}{4 \sqrt{2}}\left(T^{i} T^{j}+T^{j} T^{i}\right), \\
T_{k} & =\frac{1}{8} \epsilon_{i j k}\left(T^{i} T^{j}-T^{j} T^{i}\right) .
\end{aligned}
$$

As shown in Fig. 2, this means that $\overline{\mathbf{3}} \otimes \overline{\mathbf{3}}=\overline{\mathbf{6}} \oplus \mathbf{3}$.

In the case of $\mathbf{6} \otimes \overline{\mathbf{3}}$, we have

$$
S_{j k} T^{i}=T_{j k}^{i}+\frac{1}{\sqrt{2}}\left(\delta_{j}^{i} \delta_{k}^{m}+\delta_{k}^{i} \delta_{j}^{m}\right) Q_{m}
$$

where

$$
\begin{aligned}
Q_{m} & =\frac{1}{\sqrt{8}} S_{m l} T^{l}, \\
T_{j k}^{i} & =S_{j k} T^{i}-\frac{1}{\sqrt{2}}\left(\delta_{j}^{i} \delta_{k}^{m}+\delta_{k}^{i} \delta_{j}^{m}\right) Q_{m},
\end{aligned}
$$

which shows that $\mathbf{6} \otimes \overline{\mathbf{3}}=\mathbf{1 5} \oplus \mathbf{3}$. One can easily make sure that $T_{j k}^{i}$ represents $\mathbf{1 5}$. Namely, the 6 states from the two (symmetric) lower indices and 3 states from the upper index give 18 possible states, which are reduced to 15 when combined with three traceless conditions.

Similarly, $\overline{\mathbf{3}} \otimes \mathbf{6}$ is broken down to

$$
T^{i} S_{j k}=\widetilde{T}_{j k}^{i}+\frac{1}{\sqrt{2}}\left(\delta_{j}^{i} \delta_{k}^{m}+\delta_{k}^{i} \delta_{j}^{m}\right) \widetilde{Q}_{m}
$$


where

$$
\begin{aligned}
& \widetilde{Q}_{m}=\frac{1}{\sqrt{8}} T^{l} S_{m l}, \\
& \widetilde{T}_{j k}^{i}=T^{i} S_{j k}-\frac{1}{\sqrt{2}}\left(\delta_{j}^{i} \delta_{k}^{m}+\delta_{k}^{i} \delta_{j}^{m}\right) \widetilde{Q}_{m} .
\end{aligned}
$$

The product $\mathbf{6} \otimes \mathbf{6}$ is obtained as

$$
S_{j k} S_{l m}=\frac{1}{\sqrt{6}} T_{j k l m}+\frac{1}{2 \sqrt{2}}\left(\epsilon_{a k l} \delta_{m}^{b} \delta_{j}^{c}+\epsilon_{a j m} \delta_{l}^{b} \delta_{k}^{c}\right) S_{b c}^{a}+\frac{1}{\sqrt{6}}\left(\epsilon_{a j l} \epsilon_{b k m}+\epsilon_{a k l} \epsilon_{b j m}\right) T^{a b},
$$

where

$$
\begin{aligned}
T^{i j} & =\frac{1}{\sqrt{6}} \epsilon^{i a b} \epsilon^{j c d} S_{a c} S_{b d}, \\
S_{j k}^{i} & =\frac{1}{\sqrt{2}} \epsilon^{i l m}\left(S_{j l} S_{k m}+S_{k l} S_{j m}\right), \\
T_{j k l m} & =\frac{1}{\sqrt{6}}\left(S_{j k} S_{l m}+S_{l k} S_{j m}+S_{j m} S_{k l}+S_{l j} S_{k m}+S_{k m} S_{j l}+S_{l m} S_{j k}\right),
\end{aligned}
$$

as we have expected from $\mathbf{6} \otimes \mathbf{6}=\mathbf{1 5}_{1} \oplus \mathbf{1 5}_{2} \oplus \overline{\mathbf{6}}$. Here, we have an identity,

$$
\epsilon_{a l k} T_{j m}^{a}+\epsilon_{a k j} T_{l m}^{a}+\epsilon_{a j l} T_{k m}^{a}=0,
$$

which is valid for $T_{j k}^{i}$ in Eq. (2.12), $\widetilde{T}_{j k}^{i}$ in Eq. (2.14), and $S_{j k}^{i}$ in Eq. (2.16).

By collecting the above results, we have

$$
\begin{aligned}
\left(q_{j} q_{k}\right)\left(q_{l} q_{m}\right)= & \frac{1}{2 \sqrt{6}} T_{j k l m}+\frac{1}{4 \sqrt{2}}\left(\epsilon_{a k l} \delta_{m}^{b} \delta_{j}^{c}+\epsilon_{a j m} \delta_{l}^{b} \delta_{k}^{c}\right) S_{b c}^{a} \\
& +\frac{1}{\sqrt{6}}\left(\epsilon_{a j l} \epsilon_{b k m}+\epsilon_{a k l} \epsilon_{b j m}\right) T^{a b}+\frac{1}{4} \epsilon_{i j k}\left\{T_{l m}^{i}+\frac{1}{\sqrt{2}}\left(\delta_{l}^{i} \delta_{m}^{a}+\delta_{m}^{i} \delta_{l}^{a}\right) Q_{a}\right\} \\
& +\frac{1}{4} \epsilon_{i j k}\left\{\widetilde{T}_{l m}^{i}+\frac{1}{\sqrt{2}}\left(\delta_{l}^{i} \delta_{m}^{a}+\delta_{m}^{i} \delta_{l}^{a}\right) \widetilde{Q}_{a}\right\}+\frac{1}{4 \sqrt{2}} \epsilon_{i j k} \epsilon_{n l m}\left(2 S^{i n}+\epsilon^{i n a} T_{a}\right) .
\end{aligned}
$$

The obtained irreducible representations for four-quarks are summarized in Table II.

The inner products of the multiplets are obtained as follows. First, for $T_{a}, Q_{a}$, and $\widetilde{Q}_{a}$ of $(1,0)$ type, we have

$$
\left(T_{a}, T_{b}\right)=2 \delta_{a b}
$$

For $T^{i j}$ and $S^{i j}$ of $(0,2)$ type, we have

$$
\left(T^{i j}, T^{l m}\right)=\delta^{i l} \delta^{j m}+\delta^{i m} \delta^{j l} .
$$

For $T_{j k}^{i}, \widetilde{T}_{j k}^{i}$, and $S_{j k}^{i}$ of $(2,1)$ type, which satisfy the traceless condition, $T_{i k}^{i}=0$, we have

$$
\left(T_{j k}^{i}, T_{m n}^{l}\right)=4 \delta^{i l}\left(\delta_{j m} \delta_{k n}+\delta_{j n} \delta_{k m}\right)-\delta_{j}^{i}\left(\delta_{m}^{l} \delta_{k n}+\delta_{n}^{l} \delta_{k m}\right)-\delta_{k}^{i}\left(\delta_{m}^{l} \delta_{j n}+\delta_{n}^{l} \delta_{j m}\right) .
$$

For $T_{i j k l}$ of $(4,0)$ type, we have

$$
\begin{aligned}
\left(T_{j k l m}, T_{a b c d}\right)= & \left(S_{j k}, S_{a b}\right)\left(S_{l m}, S_{c d}\right)+\left(S_{j k}, S_{b c}\right)\left(S_{l m}, S_{a d}\right)+\left(S_{j k}, S_{a d}\right)\left(S_{l m}, S_{b c}\right) \\
& +\left(S_{j k}, S_{a c}\right)\left(S_{l m}, S_{b d}\right)+\left(S_{j k}, S_{b d}\right)\left(S_{l m}, S_{a c}\right)+\left(S_{j k}, S_{c d}\right)\left(S_{l m}, S_{a b}\right),
\end{aligned}
$$

where

$$
\left(S_{j k}, S_{l m}\right)=\delta_{j l} \delta_{k m}+\delta_{j m} \delta_{k l}
$$

as in Eq. (2.7). 


\begin{tabular}{c|c|c|c}
\hline \hline irreducible tensor & type & notation & source \\
\hline$T_{i}$ & $(1,0)$ & $\mathbf{3}$ & $\overline{\mathbf{3}} \otimes \overline{\mathbf{3}}$ \\
$Q_{i}$ & $(1,0)$ & $\mathbf{3}$ & $\mathbf{6} \otimes \overline{\mathbf{3}}$ \\
$\widetilde{Q}_{i}$ & $(1,0)$ & $\mathbf{3}$ & $\overline{\mathbf{3}} \otimes \mathbf{6}$ \\
$S^{i j}$ & $(0,2)$ & $\overline{\mathbf{6}}$ & $\overline{\mathbf{3}} \otimes \overline{\mathbf{3}}$ \\
$T^{i j}$ & $(0,2)$ & $\overline{\mathbf{6}}$ & $\mathbf{6} \otimes \mathbf{6}$ \\
$T_{j k}^{i}$ & $(2,1)$ & $\mathbf{1 5}_{2}$ & $\mathbf{6} \otimes \overline{\mathbf{3}}$ \\
$\widetilde{T}_{j k}^{i}$ & $(2,1)$ & $\mathbf{1 5}_{2}$ & $\overline{\mathbf{3}} \otimes \mathbf{6}$ \\
$S_{j k}^{i}$ & $(2,1)$ & $\mathbf{1 5}_{2}$ & $\mathbf{6} \otimes \mathbf{6}$ \\
$T_{i j k l}^{i}$ & $(4,0)$ & $\mathbf{1 5}_{1}$ & $\mathbf{6} \otimes \mathbf{6}$ \\
\hline \hline
\end{tabular}

TABLE II: Irreducible tensors of the product of two diquarks.

(a)
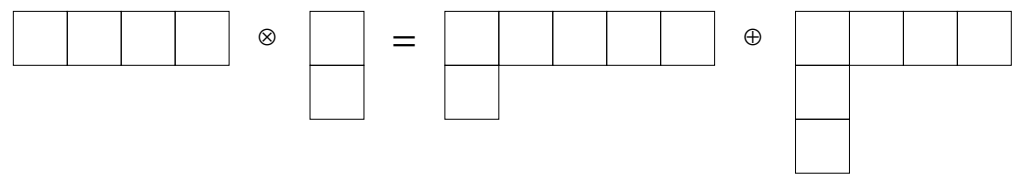

(b)
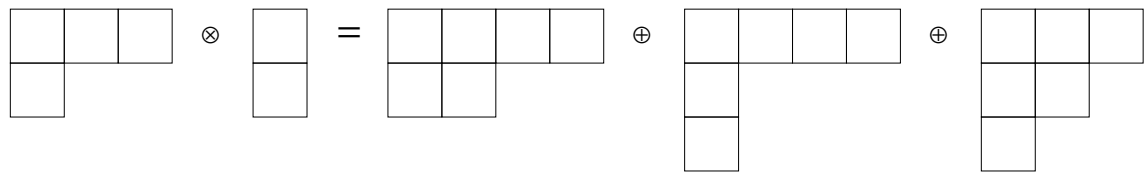

(c)
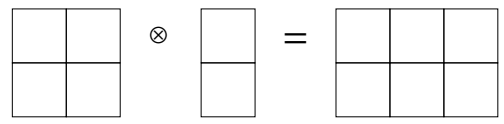

$\oplus$

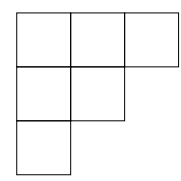

(d)
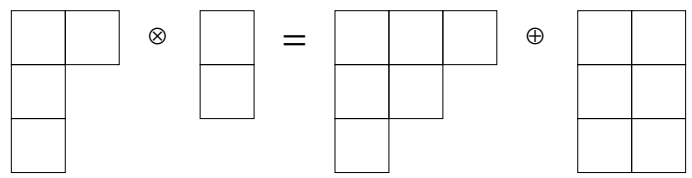

FIG. 3: Products of two diquarks and one antiquark. (a) $\mathbf{1 5}_{1} \otimes \overline{\mathbf{3}}=\mathbf{3 5} \oplus \mathbf{1 0}$, (b) $\mathbf{1 5}_{2} \otimes \overline{\mathbf{3}}=\mathbf{2 7} \oplus \mathbf{1 0} \oplus \mathbf{8},(\mathrm{c}) \overline{\mathbf{6}} \otimes \overline{\mathbf{3}}=\overline{\mathbf{1 0}} \oplus \mathbf{8}$, and $(\mathrm{d}) \mathbf{3} \otimes \overline{\mathbf{3}}=\mathbf{8} \oplus \mathbf{1}$.

\section{Pentaquarks}

As the two diquarks can form $\mathbf{3}, \overline{\mathbf{6}}, \mathbf{1 5} \mathbf{5}_{1}$, and $\mathbf{1 5}_{2}$ and the antiquarks form $\overline{\mathbf{3}}$, there are six multiplets for pentaquarks, $\mathbf{1}, \mathbf{8}, \mathbf{1 0}, \overline{\mathbf{1 0}}, \mathbf{2 7}$, and $\mathbf{3 5}$ as depicted in Fig. 3. We now discuss the possible pentaquark multiplets in detail.

\section{1. $\mathbf{3} \otimes \overline{\mathbf{3}}$}

The two diquarks can form $\mathbf{3}$ when they are in $\overline{\mathbf{3}} \otimes \overline{\mathbf{3}}$ or $\mathbf{6} \otimes \overline{\mathbf{3}}$. Since

$$
\mathbf{3} \otimes \overline{\mathbf{3}}=\mathbf{8} \oplus \mathbf{1},
$$

the pentaquarks are either in singlet or in octet representation in this case. Let $T_{i}$ stand for $T_{i}, Q_{i}$, or $\widetilde{Q}_{i}$ of $(1,0)$ type for the four-quarks, then we have

$$
T_{l} \bar{q}^{q}=\sqrt{2}\left(P_{l}^{q}+\frac{1}{\sqrt{3}} \delta_{l}^{q} S\right)
$$


where

$$
\begin{aligned}
S & =\frac{1}{\sqrt{6}} T_{m} \bar{q}^{m}, \\
P_{l}^{q} & =\frac{1}{\sqrt{2}}\left(T_{l} \bar{q}^{q}-\sqrt{\frac{2}{3}} \delta_{l}^{q} S\right) .
\end{aligned}
$$

Thus, $S$ and $P_{l}^{q}$ represent the pentaquark singlet and pentaquark octet, respectively.

$$
\text { 2. } \overline{\mathbf{6}} \otimes \overline{\mathbf{3}}
$$

When the two diquarks are in $\overline{\mathbf{3}} \otimes \overline{\mathbf{3}}$ or in $\mathbf{6} \otimes \mathbf{6}$, they can form $\overline{\mathbf{6}}$. In this case, we have antidecuplet and octet as

$$
\overline{\mathbf{6}} \otimes \overline{\mathbf{3}}=\overline{\mathbf{1 0}} \oplus \mathbf{8} .
$$

This is the only case where we can form the antidecuplet. In other words, antidecuplet baryons can be formed only when both diquarks are in $\overline{\mathbf{3}}$ or the both are in $\mathbf{6}$.

For $S^{i j}$ or $T^{i j}$ of $(0,2)$ type for the four-quark system, which we write as $S^{i j}$ in general, we have

$$
S^{i j} \bar{q}^{q}=\frac{1}{\sqrt{3}}\left\{T^{i j q}+\left(\epsilon^{l j q} P_{l}^{i}+\epsilon^{l i q} P_{l}^{j}\right)\right\}
$$

where

$$
\begin{aligned}
T^{i j k} & =\frac{1}{\sqrt{3}}\left(S^{i j} \bar{q}^{k}+S^{i k} \bar{q}^{j}+S^{j k} \bar{q}^{i}\right), \\
P_{l}^{i} & =\frac{1}{\sqrt{3}} \epsilon_{l a b} S^{i a} \bar{q}^{b},
\end{aligned}
$$

which represent the pentaquark antidecuplet and pentaquark octet, respectively.

$$
\text { 3. } 15_{1} \otimes \overline{3}
$$

If both of the two diquarks are in $\mathbf{6}$, then one can form four-quark in 15-plet of $(4,0)$ type, which is represented by $T_{i j k l}$. Since

$$
15_{1} \otimes \overline{3}=\mathbf{3 5} \oplus \mathbf{1 0},
$$

we can form pentaquark $\mathbf{3 5}$ and 10. Note also that this is the only combination that gives rise to pentaquark 35-plet. Explicitly, we have

$$
T_{i j k l} \bar{q}^{q}=T_{i j k l}^{q}+\frac{1}{\sqrt{6}}\left(\delta_{i}^{q} \delta_{j}^{b} \delta_{k}^{c} \delta_{l}^{d}+\delta_{j}^{q} \delta_{i}^{b} \delta_{k}^{c} \delta_{l}^{d}+\delta_{k}^{q} \delta_{i}^{b} \delta_{j}^{c} \delta_{l}^{d}+\delta_{l}^{q} \delta_{i}^{b} \delta_{j}^{c} \delta_{k}^{d}\right) D_{b c d}
$$

where

$$
\begin{aligned}
D_{i j k} & =\frac{1}{\sqrt{6}} T_{i j k l} \bar{q}^{l} \\
T_{i j k l}^{a} & =T_{i j k l} \bar{q}^{a}-\frac{1}{\sqrt{6}}\left(\delta_{i}^{a} D_{j k l}+\delta_{j}^{a} D_{i k l}+\delta_{k}^{a} D_{i j l}+\delta_{l}^{a} D_{i j k}\right) .
\end{aligned}
$$

$$
\text { 4. } 15_{2} \otimes \overline{3}
$$

The $15_{2}$ multiplet of two diquark system can be formed when one of the diquarks is in $\mathbf{6}$ and the other diquark in $\overline{3}$. Since

$$
15_{2} \otimes \overline{3}=\mathbf{2 7} \oplus \mathbf{1 0} \oplus \mathbf{8}
$$


the 27-plet can be formed only in this case. Explicitly, if we write $T_{k l}^{i}$ for $T_{k l}^{i}, \widetilde{T}_{k l}^{i}$, and $S_{k l}^{i}$, we obtain

$$
T_{k l}^{i} \bar{q}^{q}=\sqrt{2} T_{k l}^{i q}+\sqrt{\frac{2}{3}} \epsilon^{i q m} D_{m k l}+\frac{4}{\sqrt{15}}\left(\delta_{l}^{q} \delta_{k}^{b}+\delta_{l}^{b} \delta_{k}^{q}\right) P_{b}^{i}-\frac{1}{\sqrt{15}}\left(\delta_{l}^{i} \delta_{k}^{b}+\delta_{l}^{b} \delta_{k}^{i}\right) P_{b}^{q},
$$

where

$$
\begin{aligned}
P_{j}^{i} & =\frac{1}{\sqrt{15}} T_{j k}^{i} \bar{q}^{k}, \\
D_{j k l} & =\frac{1}{\sqrt{24}}\left(\epsilon_{j a b} T_{k l}^{a} \bar{q}^{b}+\epsilon_{k a b} T_{j l}^{a} \bar{q}^{b}+\epsilon_{l a b} T_{j k}^{a} \bar{q}^{b}\right), \\
T_{k l}^{i j} & =\frac{1}{2 \sqrt{2}}\left(T_{k l}^{i} \bar{q}^{j}+T_{k l}^{j} \bar{q}^{i}\right)-\frac{1}{10 \sqrt{2}}\left(\delta_{l}^{i} P_{k}^{j}+\delta_{l}^{j} P_{k}^{i}+\delta_{k}^{i} P_{l}^{j}+\delta_{k}^{j} P_{l}^{i}\right) .
\end{aligned}
$$

\section{Inner products}

The results for the pentaquark multiplets obtained in this Section are summarized in Table III. Using the expressions given above, it is now straightforward to check the traceless conditions, $P_{i}^{i}=T_{i k}^{i j}=T_{i j k l}^{i}=0$. Furthermore, the inner products are obtained as

$$
\begin{aligned}
&(S, S)= 1, \\
&\left(P_{j}^{i}, P_{l}^{k}\right)= \delta^{i k} \delta_{j l}-\frac{1}{3} \delta_{j}^{i} \delta_{l}^{k} \\
&\left(D_{i j k}, D_{l m n}\right)=\left(S_{i j}, S_{l m}\right) \delta_{k n}+\left(S_{i j}, S_{l n}\right) \delta_{k m}+\left(S_{i j}, S_{m n}\right) \delta_{k l}, \\
&\left(T^{i j k}, T^{l m n}\right)=\left(S^{i j}, S^{l m}\right) \delta^{k n}+\left(S^{i j}, S^{l n}\right) \delta^{k m}+\left(S^{i j}, S^{m n}\right) \delta^{k l} \\
&\left(T_{k l}^{i j}, T_{r s}^{p q}\right)=\left(S^{i j}, S^{p q}\right)\left(S_{k l}, S_{r s}\right)-\frac{1}{5}\left(\delta^{j p} \delta_{r}^{q}+\delta^{j q} \delta_{r}^{p}\right)\left(\delta_{k}^{i} \delta_{l s}+\delta_{l}^{i} \delta_{k s}\right) \\
&-\frac{1}{5}\left(\delta^{i p} \delta_{r}^{q}+\delta^{i q} \delta_{r}^{p}\right)\left(\delta_{k}^{j} \delta_{l s}+\delta_{l}^{j} \delta_{k s}\right)-\frac{1}{5}\left(\delta^{j p} \delta_{s}^{q}+\delta^{j q} \delta_{s}^{p}\right)\left(\delta_{k}^{i} \delta_{l r}+\delta_{l}^{i} \delta_{k r}\right) \\
&-\frac{1}{5}\left(\delta^{i p} \delta_{s}^{q}+\delta^{i q} \delta_{s}^{p}\right)\left(\delta_{k}^{j} \delta_{l r}+\delta_{l}^{j} \delta_{k r}\right)+\frac{1}{10}\left(\delta_{k}^{i} \delta_{l}^{j}+\delta_{l}^{i} \delta_{k}^{j}\right)\left(\delta_{r}^{p} \delta_{s}^{q}+\delta_{s}^{p} \delta_{r}^{q}\right) \\
&\left(T_{i j k l}^{a}, T_{p q r s}^{b}\right)= \delta^{a b}\left\{\left(S_{i j}, S_{p q}\right)\left(S_{k l}, S_{r s}\right)+\left(S_{i j}, S_{q r}\right)\left(S_{k l}, S_{p s}\right)+\left(S_{i j}, S_{p s}\right)\left(S_{k l}, S_{q r}\right)\right. \\
&\left.+\left(S_{i j}, S_{p r}\right)\left(S_{k l}, S_{q s}\right)+\left(S_{i j}, S_{q s}\right)\left(S_{k l}, S_{p r}\right)+\left(S_{i j}, S_{r s}\right)\left(S_{k l}, S_{p q}\right)\right\} \\
&-\frac{1}{6} \delta_{i}^{a}\left\{\delta_{p}^{b}\left(D_{j k l}, D_{q r s}\right)+\delta_{q}^{b}\left(D_{j k l}, D_{p r s}\right)+\delta_{r}^{b}\left(D_{j k l}, D_{p q s}\right)+\delta_{s}^{b}\left(D_{j k l}, D_{p q r}\right)\right\} \\
&-\frac{1}{6} \delta_{k}^{a}\left\{\delta_{p}^{b}\left(D_{i k l}, D_{q r s}\right)+\delta_{q}^{b}\left(D_{i k l}, D_{p r s}\right)+\delta_{r}^{b}\left(D_{i k l}, D_{p q s}\right)+\delta_{s}^{b}\left(D_{i k l}, D_{p q r}\right)\right\} \\
&-\frac{1}{6} \delta_{l}^{a}\left\{\delta_{p}^{b}\left(D_{i j k}, D_{q r s}\right)+\delta_{q}^{b}\left(D_{i j k}, D_{p r s}\right)+\delta_{r}^{b}\left(D_{i j l}, D_{p q s}\right)+\delta_{s}^{b}\left(D_{i j l}, D_{p q r}\right)\right\} \\
&\left.\left.D_{p q s}\right)+\delta_{s}^{b}\left(D_{i j k}, D_{p q r}\right)\right\}
\end{aligned}
$$

where

$$
\begin{aligned}
\left(S^{i j}, S^{k l}\right) & =\delta^{i k} \delta^{j l}+\delta^{i l} \delta^{j k} \\
\left(S_{i j}, S_{k l}\right) & =\delta_{i k} \delta_{j l}+\delta_{i l} \delta_{j k} .
\end{aligned}
$$

With these informations, we are ready to match the states with the physical baryons. 


\begin{tabular}{c|c|c|c}
\hline \hline irreducible tensor & type & notation & source (two diquark state) \\
\hline$S$ & $(0,0)$ & $\mathbf{1}$ & $\mathbf{3}$ \\
$P_{j}^{i}$ & $(1,1)$ & $\mathbf{8}$ & $\mathbf{3}, \overline{\mathbf{6}}, \mathbf{1 5}_{2}$ \\
$D_{i j k}$ & $(3,0)$ & $\mathbf{1 0}$ & $\mathbf{1 5}_{1}, \mathbf{1 5}_{2}$ \\
$T^{i j k}$ & $(0,3)$ & $\overline{\mathbf{1 0}}$ & $\overline{\mathbf{6}}$ \\
$T_{k l}^{i j}$ & $(2,2)$ & $\mathbf{2 7}$ & $\mathbf{1 5}_{2}$ \\
$T_{i j k l}^{a}$ & $(4,1)$ & $\mathbf{3 5}$ & $\mathbf{1 5}_{1}$ \\
\hline \hline
\end{tabular}

TABLE III: Representations of the pentaquark multiplets.

\section{FLAVOR WAVE FUNCTIONS OF PENTAQUARK BARYONS}

Now we identify the tensor representations for pentaquark baryons obtained so far with the physical baryon states. We first present our nomenclature for pentaquark particles. Based on its hypercharge, we name the baryon as

$$
\begin{array}{lc}
Y=2 & \Theta, \\
Y=1 & N, \Delta, \\
Y=0 & \Sigma, \Lambda, \\
Y=-1 & \Xi, \\
Y=-2 & \Omega, \\
Y=-3 \quad X,
\end{array}
$$

where we denote the $Y=-3$ particle as $X$ following Ref. [92]. We denote the isospin of the particle and the multiplet which it belongs to as subscripts, for example,

$$
\Sigma_{35,2}
$$

represents a particle of $\mathbf{3 5}$-plet with hypercharge 0 and isospin 2. In the case that the isospin or the multiplet is clear, we drop such subscripts as in $\Theta^{+}$. The superscript is reserved for the charge.

In tensor representation, the number of lower indices of $T_{a_{1}, \ldots, a_{p}}^{b_{1}, \ldots, b_{q}}$ is $p$ and that of upper indices is $q$. Now suppose that among its lower indices the numbers of 1's, 2's, and 3's are $p_{1}, p_{2}$, and $p_{3}$, respectively, and that among upper indices it has $q_{1}$ 1's, $q_{2} 2$ 's, and $q_{3}$ 3's. Then we have $p_{1}+p_{2}+p_{3}=p$ and $q_{1}+q_{2}+q_{3}=q$. The irreducible tensor is an eigenstate of hypercharge $Y$ and the third component of isospin $I_{3}$ with the eigenvalues [93]

$$
\begin{aligned}
Y & =p_{1}-q_{1}+p_{2}-q_{2}-\frac{2}{3}(p-q), \\
I_{3} & =\frac{1}{2}\left(p_{1}-q_{1}\right)-\frac{1}{2}\left(p_{2}-q_{2}\right) .
\end{aligned}
$$

The charge of the particle is obtained from the Gell-Mann-Nishijima formula, $Q=I_{3}+Y / 2$. By this way, we can match the $\mathrm{SU}(3)$ tensors to the physical baryon states. We summarize the pentaquark particles in Table IV.

\section{A. Singlet}

The pentaquark singlet is given by

$$
S=\frac{1}{\sqrt{6}} T_{m} \bar{q}^{m}
$$

which is normalized as $(S, S)=1$, where $T_{m}$ stands for $T_{m}, Q_{m}$, and $\widetilde{Q}_{m}$ defined in Eqs. (2.10), (2.12), and (2.14), respectively. This is identified as

$$
S=-\Lambda_{1}^{0},
$$

where the phase is chosen to be consistent with the tables of de Swart [94], i.e., the conventional phase. 


\begin{tabular}{|c|c|c|c|}
\hline multiplet & hypercharge & isospin & particle \\
\hline 1 & 0 & 0 & $\Lambda_{1}^{0}$ \\
\hline \multirow[t]{4}{*}{8} & 1 & $1 / 2$ & $N_{8}^{+}, N_{8}^{0}$ \\
\hline & 0 & 1 & $\Sigma_{8}^{+}, \Sigma_{8}^{0}, \Sigma_{8}^{-}$ \\
\hline & 0 & 0 & $\Lambda_{8}^{0}$ \\
\hline & -1 & $1 / 2$ & $\Xi_{8}^{0}, \Xi_{8}^{-}$ \\
\hline \multirow[t]{4}{*}{10} & 1 & $3 / 2$ & $\Delta_{10}^{++}, \Delta_{10}^{+}, \Delta_{10}^{0}, \Delta_{10}^{-}$ \\
\hline & 0 & 1 & $\Sigma_{10}^{+}, \Sigma_{10}^{0}, \Sigma_{10}^{-}$ \\
\hline & -1 & $1 / 2$ & $\Xi_{10}^{0}, \Xi_{10}^{-}$ \\
\hline & -2 & 0 & $\Omega_{10}^{-}$ \\
\hline \multirow[t]{4}{*}{$\overline{\overline{10}}$} & 2 & 0 & $\Theta^{+}$ \\
\hline & 1 & $1 / 2$ & $N_{\frac{+}{10}}^{+}, N \frac{0}{10}$ \\
\hline & 0 & 1 & $\Sigma_{\frac{10}{10}}^{+}, \Sigma_{\overline{10}}^{0}, \Sigma_{\overline{10}}^{-}$ \\
\hline & -1 & $3 / 2$ & $\Xi_{10,3 / 2}^{+}, \Xi_{10,3 / 2}^{0}, \Xi_{10,3 / 2}^{-}, \Xi_{\overline{10}, 3 / 2}^{--}$ \\
\hline \multirow[t]{9}{*}{27} & 2 & 1 & $\Theta_{1}^{++}, \Theta_{1}^{+}, \Theta_{1}^{0}$ \\
\hline & 1 & $3 / 2$ & $\Delta_{27}^{++}, \Delta_{27}^{+}, \Delta_{27}^{0}, \Delta_{27}^{-}$ \\
\hline & 1 & $1 / 2$ & $N_{27}^{+}, N_{27}^{0}$ \\
\hline & 0 & 2 & $\Sigma_{27,2}^{++}, \Sigma_{27,2}^{+}, \Sigma_{27,2}^{0}, \Sigma_{27,2}^{-}, \Sigma_{27,2}^{--}$ \\
\hline & 0 & 1 & $\Sigma_{27}^{+}, \Sigma_{27}^{0}, \Sigma_{27}^{-}$ \\
\hline & 0 & 0 & $\Lambda_{27}^{0}$ \\
\hline & -1 & $3 / 2$ & $\Xi_{27,3 / 2}^{+}, \Xi_{27,3 / 2}^{0}, \Xi_{27,3 / 2}^{-}, \Xi_{27,3 / 2}^{--}$ \\
\hline & -1 & $1 / 2$ & $\Xi_{27}^{0}, \Xi_{27}^{-}$ \\
\hline & -2 & 1 & $\Omega_{27,1}^{0}, \Omega_{27,1}^{-}, \Omega_{27,1}^{--}$ \\
\hline \multirow[t]{10}{*}{35} & 2 & 2 & $\Theta_{2}^{+++}, \Theta_{2}^{++}, \Theta_{2}^{+}, \Theta_{2}^{0}, \Theta_{2}^{-}$ \\
\hline & 1 & $5 / 2$ & $\Delta_{5 / 2}^{+++}, \Delta_{5 / 2}^{++}, \Delta_{5 / 2}^{+}, \Delta_{5 / 2}^{0}, \Delta_{5 / 2}^{-}, \Delta_{5 / 2}^{--}$ \\
\hline & 1 & $3 / 2$ & $\Delta_{35}^{++}, \Delta_{35}^{+}, \Delta_{35}^{0}, \Delta_{35}^{-}$ \\
\hline & 0 & 2 & $\Sigma_{35,2}^{++}, \Sigma_{35,2}^{+}, \Sigma_{35,2}^{0}, \Sigma_{35,2}^{-}, \Sigma_{35,2}^{--}$ \\
\hline & 0 & 1 & $\Sigma_{35}^{+}, \Sigma_{35}^{0}, \Sigma_{35}^{-}$ \\
\hline & -1 & $3 / 2$ & $\Xi_{35,3 / 2}^{+}, \Xi_{35,3 / 2}^{0}, \Xi_{35,3 / 2}^{-}, \Xi_{35,3 / 2}^{--}$ \\
\hline & -1 & $1 / 2$ & $\Xi_{35}^{0}, \Xi_{35}^{-}$ \\
\hline & -2 & 1 & $\Omega_{35,1}^{0}, \Omega_{35,1}^{-}, \Omega_{35,1}^{--}$ \\
\hline & -2 & 0 & $\Omega_{35}^{-}$ \\
\hline & -3 & $1 / 2$ & $X^{-}, X^{--}$ \\
\hline
\end{tabular}

TABLE IV: Pentaquark baryons

\section{B. Octet}

The weight diagram for pentaquark octet is shown in Fig. 4. The octet tensor $P_{j}^{i}$ then represent the particles as follows,

$$
\begin{aligned}
& P_{1}^{3}=N_{8}^{+}, \quad P_{2}^{3}=N_{8}^{0}, \quad P_{1}^{2}=\Sigma_{8}^{+}, \\
& P_{2}^{1}=\Sigma_{8}^{-}, \quad P_{1}^{1}=1 / \sqrt{2} \Sigma_{8}^{0}+1 / \sqrt{6} \Lambda_{8}^{0}, \quad P_{2}^{2}=-1 / \sqrt{2} \Sigma_{8}^{0}+1 / \sqrt{6} \Lambda_{8}^{0}, \\
& P_{3}^{3}=-\sqrt{2 / 3} \Lambda_{8}^{0}, \quad P_{3}^{2}=\Xi_{8}^{0}, \quad P_{3}^{1}=-\Xi_{8}^{-} .
\end{aligned}
$$

The mixed states $P_{1}^{1}, P_{2}^{2}$, and $P_{3}^{3}$ are decomposed of the physical states as follows [93, 95, 96]. This is well-known, but here we give the procedure as a pedagogic example. First, we note that the inner product of the octet members is

$$
\left(P_{j}^{i}, P_{l}^{k}\right)=\delta^{i k} \delta_{j l}-\frac{1}{3} \delta_{j}^{i} \delta_{l}^{k} .
$$

With this normalization, we can see that all the off-diagonal components are already normalized to one. For diagonal 


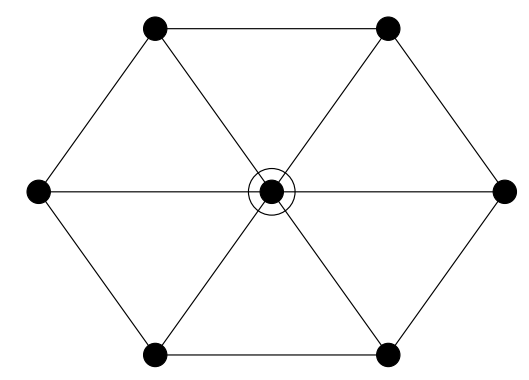

FIG. 4: Weight diagram for pentaquark octet.

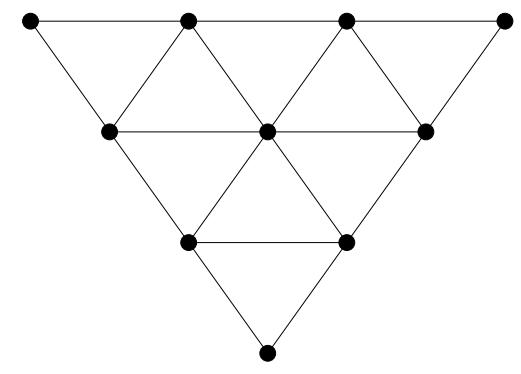

FIG. 5: Weight diagram for pentaquark decuplet.

components, we have

$$
\begin{aligned}
& \left(P_{1}^{1}, P_{1}^{1}\right)=\left(P_{2}^{2}, P_{2}^{2}\right)=\left(P_{3}^{3}, P_{3}^{3}\right)=\frac{2}{3}, \\
& \left(P_{1}^{1}, P_{2}^{2}\right)=\left(P_{2}^{2}, P_{3}^{3}\right)=\left(P_{3}^{3}, P_{1}^{1}\right)=-\frac{1}{3} .
\end{aligned}
$$

Here, one can find that $P_{3}^{3}$ does not contain $\Sigma_{8}^{0}$ part. This can be seen from the fact that by isospin lowering or raising, $P_{3}^{3}$ cannot be obtained from $\Sigma_{8}^{+}$or $\Sigma_{8}^{-}$, which are $P_{1}^{2}$ and $P_{2}^{1}$. Then we have

$$
P_{3}^{3}=-\sqrt{\frac{2}{3}} \Lambda_{8}^{0}
$$

with the conventional sign choice. In order to know $P_{1}^{1}$ and $P_{2}^{2}$, we write

$$
P_{1}^{1}=a_{1} \Sigma_{8}^{0}+b_{1} \Lambda_{8}^{0}, \quad P_{2}^{2}=a_{2} \Sigma_{8}^{0}+b_{2} \Lambda_{8}^{0} .
$$

Then the traceless condition reads

$$
a_{1}+a_{2}=0, \quad b_{1}+b_{2}-\sqrt{\frac{2}{3}}=0 .
$$

With the above condition and taking the inner products, one can obtain that

$$
a_{1}=-a_{2}=1 / \sqrt{2}, \quad b_{1}=b_{2}=1 / \sqrt{6} .
$$

Note that the phase convention for $a_{1,2}$ is chosen to be consistent with the tables of de Swart [94].

\section{Decuplet}

In the case of decuplet, we have

$$
\begin{array}{llrl}
D_{111} & =\sqrt{6} \Delta_{10}^{++}, & D_{112}=\sqrt{2} \Delta_{10}^{+}, & D_{122}=\sqrt{2} \Delta_{10}^{0}, \\
D_{222}=\sqrt{6} \Delta_{10}^{-}, & D_{113}=\sqrt{2} \Sigma_{10}^{+}, & D_{123}=-\Sigma_{10}^{0}, \\
D_{223}=-\sqrt{2} \Sigma_{10}^{-}, & D_{133}=\sqrt{2} \Xi_{10}^{0}, & D_{233}=\sqrt{2} \Xi_{10}^{-}, \\
D_{333}=-\sqrt{6} \Omega_{10}^{-} . & &
\end{array}
$$

Its weight diagram is shown in Fig. 5 . 


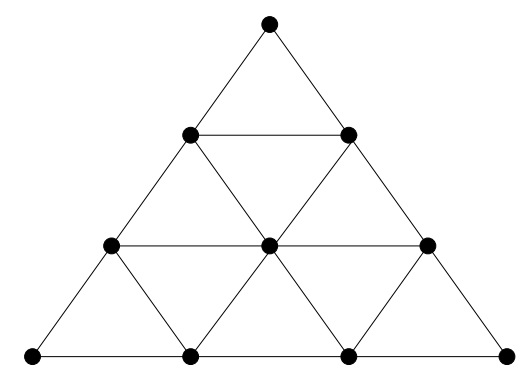

FIG. 6: Weight diagram for pentaquark antidecuplet.

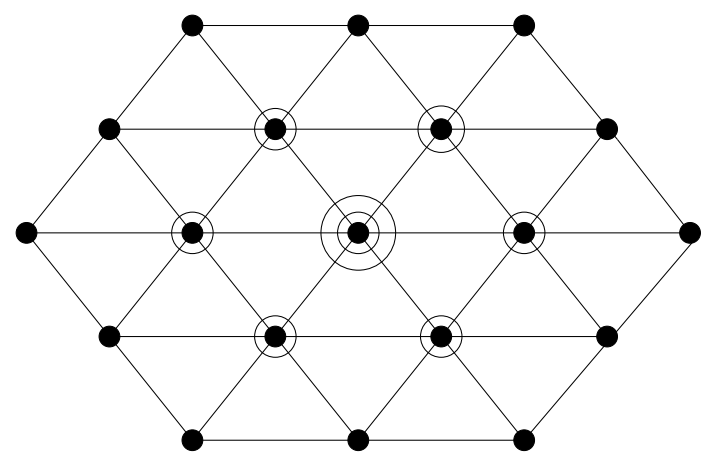

FIG. 7: Weight diagram for pentaquark 27-plet.

\section{Antidecuplet}

As can be seen from its weight diagram (Fig. 6), antidecuplet wave functions can also be read from those for decuplet baryons. We have

$$
\begin{aligned}
& T^{111}=\sqrt{6} \Xi_{\overline{10}, 3 / 2}^{--}, \quad T^{112}=-\sqrt{2} \Xi_{\overline{10}, 3 / 2}^{-}, \quad T^{122}=\sqrt{2} \Xi_{\overline{10}, 3 / 2}^{0}, \\
& T^{222}=-\sqrt{6} \Xi_{\frac{+}{10}, 3 / 2}^{+}, \quad T^{113}=\sqrt{2} \Sigma_{\overline{10}}^{-}, \quad T^{123}=-\Sigma_{\overline{10}}^{0}, \\
& T^{223}=-\sqrt{2} \Sigma_{\overline{10}}^{+}, \quad T^{133}=\sqrt{2} N_{\overline{10}}^{0}, \quad T^{233}=-\sqrt{2} N_{\frac{+}{10}}^{+}, \\
& T^{333}=\sqrt{6} \Theta^{+} .
\end{aligned}
$$

The observed $\Theta(1540)$ is identified as a member of antidecuplet. Although it has to be confirmed by other experiments, $\Xi(1862)$ is interpreted as $\Xi_{\overline{10}, 3 / 2}$. The nucleon analog $N_{\overline{10}}$, however, is not identified yet. Several ideas which suggest $N(1710)$ or $N(1440)$ as a pure antidecuplet [46] or a mixture of octet and antidecuplet [60] have been advocated. However, $N(1710)$ cannot be a pure antidecuplet member because of its large coupling to $\Delta \pi$ [61], and OZI rule in the ideally mixed pentaquark octet and antidecuplet also prohibits the coupling of the nucleon analog with $\Delta \pi[62,63]$, which excludes $N(1440)$ as a pentaquark state. If this is true, the nucleon analog $N_{8}$ or $N_{\overline{10}}$ has not been found yet. ${ }^{4}$ In Ref. [97], a possible way to search for $\Sigma_{\overline{10}}$ is discussed.

\section{E. 27-plet}

As can be read from its weight diagram (Fig. 7), the 27-plet contains two $Y=1$ baryons with isospin $1 / 2$ and $3 / 2$, three $Y=0$ baryons with isospin 2,1 , and 0 , and two $Y=-1$ baryons with isospin $1 / 2$ and $3 / 2$. Such mixed states can be classified by the method described in the previous subsection with the inner products of pentaquark states obtained in Sect. II. As an example, let us consider the $Y=0$ and $I_{3}=0$ states, which are $T_{11}^{11}, T_{12}^{12}, T_{13}^{13}, T_{22}^{22}, T_{23}^{23}$,

\footnotetext{
${ }^{4}$ It is interesting to note that SPHINX Collaboration claimed the existence of $X(2000)$ which has hidden strangeness [42].
} 
$T_{33}^{33}$. These can be written in terms of the physical states, $\Sigma_{27,2}^{0}, \Sigma_{27}^{0}$, and $\Lambda_{27}^{0}$, as

$$
\begin{aligned}
& T_{11}^{11}=a_{1} \Sigma_{27,2}^{0}+b_{1} \Sigma_{27}^{0}+c_{1} \Lambda_{27}, \\
& T_{12}^{12}=a_{2} \Sigma_{27,2}^{0}+b_{2} \Sigma_{27}^{0}+c_{2} \Lambda_{27}, \\
& T_{13}^{13}=a_{3} \Sigma_{27,2}^{0}+b_{3} \Sigma_{27}^{0}+c_{3} \Lambda_{27}, \\
& T_{22}^{22}=a_{4} \Sigma_{27,2}^{0}+b_{4} \Sigma_{27}^{0}+c_{4} \Lambda_{27}, \\
& T_{23}^{23}=a_{5} \Sigma_{27,2}^{0}+b_{5} \Sigma_{27}^{0}+c_{5} \Lambda_{27}, \\
& T_{33}^{33}=a_{6} \Sigma_{27,2}^{0}+b_{6} \Sigma_{27}^{0}+c_{6} \Lambda_{27}
\end{aligned}
$$

First, we note that $T_{33}^{33}$ has isospin 0 . So we have $a_{6}=b_{6}=0$. The inner product relation (2.40) gives

$$
\left(T_{33}^{33}, T_{33}^{33}\right)=\frac{6}{5}
$$

which leads to $c_{6}=\sqrt{\frac{6}{5}}$. We also have the inner products,

$$
\begin{array}{ll}
\left(T_{11}^{11}, T_{33}^{33}\right)=\frac{2}{5}, & \left(T_{12}^{12}, T_{33}^{33}\right)=\frac{1}{5}, \quad\left(T_{13}^{13}, T_{33}^{33}\right)=-\frac{3}{5}, \\
\left(T_{22}^{22}, T_{33}^{33}\right)=\frac{2}{5}, & \left(T_{23}^{23}, T_{33}^{33}\right)=-\frac{3}{5},
\end{array}
$$

which allow us to fix the values of $c_{i}$. The other inner products are

$$
\begin{array}{llll}
\left(T_{11}^{11}, T_{11}^{11}\right)=\frac{6}{5}, & \left(T_{12}^{12}, T_{12}^{12}\right)=\frac{7}{10}, & \left(T_{13}^{13}, T_{13}^{13}\right)=\frac{7}{10}, & \left(T_{22}^{22}, T_{22}^{22}\right)=\frac{6}{5}, \\
\left(T_{23}^{23}, T_{23}^{23}\right)=\frac{7}{10}, & \left(T_{11}^{11}, T_{12}^{12}\right)=-\frac{3}{5}, & \left(T_{11}^{11}, T_{13}^{13}\right)=-\frac{3}{5}, & \left(T_{11}^{11}, T_{22}^{22}\right)=\frac{2}{5}, \\
\left(T_{11}^{11}, T_{23}^{23}\right)=\frac{1}{5}, & \left(T_{12}^{12}, T_{13}^{13}\right)=-\frac{1}{10}, & \left(T_{12}^{12}, T_{22}^{22}\right)=-\frac{3}{5}, & \left(T_{12}^{12}, T_{23}^{23}\right)=-\frac{1}{10}, \\
\left(T_{13}^{13}, T_{22}^{22}\right)=\frac{1}{5}, & \left(T_{13}^{13}, T_{23}^{23}\right)=-\frac{1}{10}, & \left(T_{22}^{22}, T_{23}^{23}\right)=-\frac{3}{5} . &
\end{array}
$$

Together with the traceless condition $T_{i k}^{i j}=0$, which gives

$$
a_{1}+a_{2}+a_{3}=a_{2}+a_{4}+a_{5}=b_{1}+b_{2}+b_{3}=b_{2}+b_{4}+b_{5}=0,
$$

and the fact that $T_{13}^{23}$ is the $\Sigma_{27}^{+}$state, which implies that $T_{12}^{12}$ does not contain $\Sigma_{27}^{0}$ state as it cannot be obtained from $T_{13}^{23}$ by isospin lowering operator, the above relations fix the constants $a_{i}, b_{i}$, and $c_{i}$ as given below.

After taking the conventional phase choice [94], we obtain as follows.

- $Y=2, I=1$

$$
T_{11}^{33}=-2 \Theta_{1}^{++}, \quad T_{12}^{33}=\sqrt{2} \Theta_{1}^{+}, \quad T_{22}^{33}=2 \Theta_{1}^{0},
$$

- $Y=1, I=3 / 2,1 / 2$

$$
\begin{aligned}
T_{11}^{23} & =-\sqrt{2} \Delta_{27}^{++}, & T_{11}^{13} & =\sqrt{\frac{2}{3}} \Delta_{27}^{+}+\sqrt{\frac{8}{15}} N_{27}^{+}, & T_{12}^{23} & =-\sqrt{\frac{2}{3}} \Delta_{27}^{+}+\sqrt{\frac{2}{15}} N_{27}^{+}, \\
T_{13}^{33} & =-\sqrt{\frac{6}{5}} N_{27}^{+}, & T_{12}^{13} & =\sqrt{\frac{2}{3}} \Delta_{27}^{0}+\sqrt{\frac{2}{15}} N_{27}^{0}, & T_{22}^{23} & =-\sqrt{\frac{2}{3}} \Delta_{27}^{0}+\sqrt{\frac{8}{15}} N_{27}^{0}, \\
T_{23}^{33} & =-\sqrt{\frac{6}{5}} N_{27}^{0}, & T_{22}^{13} & =\sqrt{2} \Delta_{27}^{-}, & &
\end{aligned}
$$




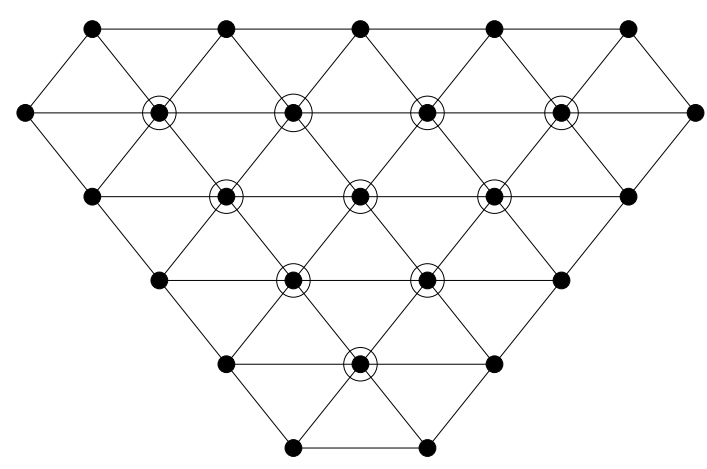

FIG. 8: Weight diagram for pentaquark 35-plet.

- $Y=0, I=2,1,0$

$$
\begin{aligned}
& T_{11}^{22}=2 \Sigma_{27,2}^{++}, \quad T_{11}^{12}=-\Sigma_{27,2}^{+}+\frac{1}{\sqrt{5}} \Sigma_{27}^{+}, \quad T_{12}^{22}=\Sigma_{27,2}^{+}+\frac{1}{\sqrt{5}} \Sigma_{27}^{+}, \\
& T_{13}^{23}=-\frac{2}{\sqrt{5}} \Sigma_{27}^{+}, \quad T_{11}^{11}=\sqrt{\frac{2}{3}} \Sigma_{27,2}^{0}+\sqrt{\frac{2}{5}} \Sigma_{27}^{0}+\sqrt{\frac{2}{15}} \Lambda_{27}^{0}, \\
& T_{12}^{12}=-\sqrt{\frac{2}{3}} \Sigma_{27,2}^{0}+\frac{1}{\sqrt{30}} \Lambda_{27}^{0}, \quad T_{13}^{13}=-\sqrt{\frac{2}{5}} \Sigma_{27}^{0}-\sqrt{\frac{3}{10}} \Lambda_{27}^{0}, \\
& T_{22}^{22}=\sqrt{\frac{2}{3}} \Sigma_{27,2}^{0}-\sqrt{\frac{2}{5}} \Sigma_{27}^{0}+\sqrt{\frac{2}{15}} \Lambda_{27}^{0}, \quad T_{23}^{23}=\sqrt{\frac{2}{5}} \Sigma_{27}^{0}-\sqrt{\frac{3}{10}} \Lambda_{27}^{0}, \\
& T_{33}^{33}=\sqrt{\frac{6}{5}} \Lambda_{27}^{0}, \quad T_{12}^{11}=\Sigma_{27,2}^{-}+\sqrt{\frac{1}{5}} \Sigma_{27}^{-}, \quad T_{22}^{12}=-\Sigma_{27,2}^{-}+\sqrt{\frac{1}{5}} \Sigma_{27}^{-}, \\
& T_{23}^{13}=-\frac{2}{\sqrt{5}} \Sigma_{27}^{-}, \quad T_{22}^{11}=2 \Sigma_{27,2}^{--},
\end{aligned}
$$

- $Y=-1, I=3 / 2,1 / 2$

$$
\begin{aligned}
& T_{13}^{22}=-\sqrt{2} \Xi_{27,3 / 2}^{+}, \quad T_{13}^{12}=\sqrt{\frac{2}{3}} \Xi_{27,3 / 2}^{0}+\sqrt{\frac{2}{15}} \Xi_{27}^{0}, \\
& T_{23}^{22}=-\sqrt{\frac{2}{3}} \Xi_{27,3 / 2}^{0}+\sqrt{\frac{8}{15}} \Xi_{27}^{0}, \quad T_{33}^{23}=-\sqrt{\frac{6}{5}} \Xi_{27}^{0}, \\
& T_{13}^{11}=-\sqrt{\frac{2}{3}} \Xi_{27,3 / 2}^{-}-\sqrt{\frac{8}{15}} \Xi_{27}^{-}, \quad T_{23}^{12}=\sqrt{\frac{2}{3}} \Xi_{27,3 / 2}^{-}-\sqrt{\frac{2}{15}} \Xi_{27}^{-}, \\
& T_{33}^{13}=\sqrt{\frac{6}{5}} \Xi_{27}^{-}, \quad T_{23}^{11}=-\sqrt{2} \Xi_{27,3 / 2}^{--},
\end{aligned}
$$

- $Y=-2, I=1$

$$
T_{33}^{22}=-2 \Omega_{27,1}^{0}, \quad T_{33}^{12}=-\sqrt{2} \Omega_{27,1}^{-}, \quad T_{33}^{11}=2 \Omega_{27,1}^{--} .
$$

\section{F. 35-plet}

The weight diagram for 35-plet is given in Fig. 8 and the members are identified as follows.

- $Y=2, I=2$

$$
\begin{aligned}
& T_{1111}^{3}=2 \sqrt{6} \Theta_{2}^{+++}, \quad T_{1112}^{3}=\sqrt{6} \Theta_{2}^{++}, \quad T_{1122}^{3}=2 \Theta_{2}^{+}, \\
& T_{1222}^{3}=\sqrt{6} \Theta_{2}^{0}, \quad T_{2222}^{3}=2 \sqrt{6} \Theta_{2}^{-},
\end{aligned}
$$


- $Y=1, I=5 / 2,3 / 2$

$$
\begin{aligned}
& T_{1111}^{2}=-2 \sqrt{6} \Delta_{5 / 2}^{+++}, \quad T_{1111}^{1}=2 \sqrt{\frac{6}{5}} \Delta_{5 / 2}^{++}+\frac{4}{\sqrt{5}} \Delta_{35}^{++}, \\
& T_{1112}^{2}=-2 \sqrt{\frac{6}{5}} \Delta_{5 / 2}^{++}+\frac{1}{\sqrt{5}} \Delta_{35}^{++}, \quad T_{1113}^{3}=-\sqrt{5} \Delta_{35}^{++} \\
& T_{1112}^{1}=2 \sqrt{\frac{3}{5}} \Delta_{5 / 2}^{+}+\sqrt{\frac{3}{5}} \Delta_{35}^{+}, \quad T_{1122}^{2}=-2 \sqrt{\frac{3}{5}} \Delta_{5 / 2}^{+}+\frac{2}{\sqrt{15}} \Delta_{35}^{+}, \\
& T_{1123}^{3}=-\sqrt{\frac{5}{3}} \Delta_{35}^{+}, \quad T_{1122}^{1}=2 \sqrt{\frac{3}{5}} \Delta_{5 / 2}^{0}+\frac{2}{\sqrt{15}} \Delta_{35}^{0}, \\
& T_{1222}^{2}=-2 \sqrt{\frac{3}{5}} \Delta_{5 / 2}^{0}+\sqrt{\frac{3}{5}} \Delta_{35}^{0}, \quad T_{1223}^{3}=-\sqrt{\frac{5}{3}} \Delta_{35}^{0}, \\
& T_{1222}^{1}=2 \sqrt{\frac{6}{5}} \Delta_{5 / 2}^{-}+\frac{1}{\sqrt{5}} \Delta_{35}^{-}, \quad T_{2222}^{2}=-2 \sqrt{\frac{6}{5}} \Delta_{5 / 2}^{-}+\frac{4}{\sqrt{5}} \Delta_{35}^{-}, \\
& T_{2223}^{3}=-\sqrt{5} \Delta_{35}^{-}, \quad T_{2222}^{1}=2 \sqrt{6} \Delta_{5 / 2}^{--}
\end{aligned}
$$

- $Y=0, I=2,1$

$$
\begin{aligned}
& T_{1113}^{2}=-\sqrt{6} \Sigma_{35,2}^{++}, \quad T_{1113}^{1}=\sqrt{\frac{3}{2}} \Sigma_{35,2}^{+}+\sqrt{\frac{3}{2}} \Sigma_{35}^{+}, \\
& T_{1123}^{2}=-\sqrt{\frac{3}{2}} \Sigma_{35,2}^{+}+\sqrt{\frac{1}{6}} \Sigma_{35}^{+}, \quad T_{1133}^{3}=-\sqrt{\frac{8}{3}} \Sigma_{35}^{+}, \\
& T_{1123}^{1}=\Sigma_{35,2}^{0}+\sqrt{\frac{1}{3}} \Sigma_{35}^{0}, \quad T_{1223}^{2}=-\Sigma_{35,2}^{0}+\sqrt{\frac{1}{3}} \Sigma_{35}^{0}, \\
& T_{1233}^{3}=-\sqrt{\frac{4}{3}} \Sigma_{35}^{0}, \quad T_{1223}^{1}=\sqrt{\frac{3}{2}} \Sigma_{35,2}^{-}+\sqrt{\frac{1}{6}} \Sigma_{35}^{-}, \\
& T_{2223}^{2}=-\sqrt{\frac{3}{2}} \Sigma_{35,2}^{-}+\sqrt{\frac{3}{2}} \Sigma_{35}^{-}, \quad T_{2233}^{3}=-\sqrt{\frac{8}{3}} \Sigma_{35}^{-}, \\
& T_{2223}^{1}=\sqrt{6} \Sigma_{35,2}^{--} .
\end{aligned}
$$

- $Y=-1, I=3 / 2,1 / 2$

$$
\begin{aligned}
& T_{1133}^{2}=-2 \Xi_{35,3 / 2}^{+}, \quad T_{1133}^{1}=\sqrt{\frac{4}{3}} \Xi_{35,3 / 2}^{0}+\sqrt{\frac{4}{3}} \Xi_{35}^{0}, \\
& T_{1233}^{2}=-\sqrt{\frac{4}{3}} \Xi_{35,3 / 2}^{0}+\sqrt{\frac{1}{3}} \Xi_{35}^{0}, \quad T_{1333}^{3}=-\sqrt{3} \Xi_{35}^{0}, \\
& T_{1233}^{1}=\sqrt{\frac{4}{3}} \Xi_{35,3 / 2}^{-}+\sqrt{\frac{1}{3}} \Xi_{35}^{-}, \quad T_{2233}^{2}=-\sqrt{\frac{4}{3}} \Xi_{35,3 / 2}^{-}+\sqrt{\frac{4}{3}} \Xi_{35}^{-}, \\
& T_{2333}^{3}=-\sqrt{3} \Xi_{35}^{-}, \quad T_{2233}^{1}=2 \Xi_{35,3 / 2}^{--}
\end{aligned}
$$

- $Y=-2, I=1,0$

$$
\begin{aligned}
& T_{1333}^{2}=-\sqrt{6} \Omega_{35,1}^{0}, \quad T_{1333}^{1}=-\sqrt{3} \Omega_{35,1}^{-}-\sqrt{2} \Omega_{35}^{-}, \\
& T_{2333}^{2}=\sqrt{3} \Omega_{35,1}^{-}-\sqrt{2} \Omega_{35}^{-}, \quad T_{3333}^{3}=2 \sqrt{2} \Omega_{35}^{-}, \quad T_{2333}^{1}=-\sqrt{6} \Omega_{35,1}^{--} .
\end{aligned}
$$

- $Y=-3, I=1 / 2$

$$
T_{3333}^{2}=-\sqrt{24} X^{-}, \quad T_{3333}^{1}=\sqrt{24} X^{--} .
$$

As we have identified the pentaquark irreducible tensors with the physical baryon states, it is straightforward to obtain their flavor wave functions. For example, the flavor wave function of the singlet state, $\Lambda_{1}^{0}$, is obtained as

$$
\Lambda_{1}^{0}=-\frac{c_{1}}{\sqrt{6}} T_{m} \bar{q}^{m}-\frac{c_{2}}{\sqrt{6}} Q_{m} \bar{q}^{m}-\frac{c_{3}}{\sqrt{6}} \widetilde{Q}_{m} \bar{q}^{m},
$$


where $T_{m}, Q_{m}$, and $\widetilde{Q}_{m}$ are defined in Eqs. (2.10), (2.12), and (2.14). By noting that $q_{1}=u, q_{2}=d$, and $q_{3}=s$, one can explicitly write down the wave function in terms of quark flavors. The coefficients $c_{i}$ are constrained as $c_{1}^{2}+c_{2}^{2}+c_{3}^{2}=1$. The octet, however, has eight coefficients as

$$
\begin{aligned}
P_{j}^{i}= & \frac{c_{1}}{\sqrt{2}}\left(T_{j} \bar{q}^{i}-\frac{1}{3} \delta_{j}^{i} T_{m} \bar{q}^{m}\right)+\frac{c_{2}}{\sqrt{2}}\left(Q_{j} \bar{q}^{i}-\frac{1}{3} \delta_{j}^{i} Q_{m} \bar{q}^{m}\right)+\frac{c_{3}}{\sqrt{2}}\left(\widetilde{Q}_{j} \bar{q}^{i}-\frac{1}{3} \delta_{j}^{i} \widetilde{Q}_{m} \bar{q}^{m}\right) \\
& +\frac{c_{4}}{\sqrt{3}} \epsilon_{j a b} S^{i a} \bar{q}^{b}+\frac{c_{5}}{\sqrt{3}} \epsilon_{j a b} T^{i a} \bar{q}^{b}+\frac{c_{6}}{\sqrt{15}} T_{j k}^{i} \bar{q}^{k}+\frac{c_{7}}{\sqrt{15}} \widetilde{T}_{j k}^{i} \bar{q}^{k}+\frac{c_{8}}{\sqrt{15}} S_{j k}^{i} \bar{q}^{k} .
\end{aligned}
$$

The flavor wave functions of other multiplets are obtained as

$$
\begin{aligned}
D_{i j k}= & \frac{c_{1}}{\sqrt{6}} T_{i j k l} \bar{q}^{l}+\frac{c_{2}}{\sqrt{24}}\left(\epsilon_{i a b} T_{j k}^{a} \bar{q}^{b}+\epsilon_{j a b} T_{k i}^{a} \bar{q}^{b}+\epsilon_{k a b} T_{i j}^{a} \bar{q}^{b}\right) \\
& +\frac{c_{3}}{\sqrt{24}}\left(\epsilon_{i a b} \widetilde{T}_{j k}^{a} \bar{q}^{b}+\epsilon_{j a b} \widetilde{T}_{k i}^{a} \bar{q}^{b}+\epsilon_{k a b} \widetilde{T}_{i j}^{a} \bar{q}^{b}\right)+\frac{c_{4}}{\sqrt{24}}\left(\epsilon_{i a b} S_{j k}^{a} \bar{q}^{b}+\epsilon_{j a b} S_{k i}^{a} \bar{q}^{b}+\epsilon_{k a b} S_{i j}^{a} \bar{q}^{b}\right), \\
T^{i j k}= & \frac{c_{1}}{\sqrt{3}}\left(S^{i j} \bar{q}^{k}+S^{j k} \bar{q}^{i}+S^{k i} \bar{q}^{j}\right)+\frac{c_{2}}{\sqrt{3}}\left(T^{i j} \bar{q}^{k}+T^{j k} \bar{q}^{i}+T^{k i} \bar{q}^{j}\right), \\
T_{k l}^{i j}= & c_{1}\left\{\frac{1}{2 \sqrt{2}}\left(T_{k l}^{i} \bar{q}^{j}+T_{k l}^{j} \bar{q}^{i}\right)-\frac{1}{10 \sqrt{30}}\left(\delta_{l}^{i} T_{k m}^{j} \bar{q}^{m}+\delta_{l}^{j} T_{k m}^{i} \bar{q}^{m}+\delta_{k}^{i} T_{l m}^{j} \bar{q}^{m}+\delta_{k}^{j} T_{l m}^{i} \bar{q}^{m}\right)\right\} \\
& +c_{2}\left\{\frac{1}{2 \sqrt{2}}\left(\widetilde{T}_{k l}^{i} \bar{q}^{j}+\widetilde{T}_{k l}^{j} \bar{q}^{i}\right)-\frac{1}{10 \sqrt{30}}\left(\delta_{l}^{i} \widetilde{T}_{k m}^{j} \bar{q}^{m}+\delta_{l}^{j} \widetilde{T}_{k m}^{i} \bar{q}^{m}+\delta_{k}^{i} \widetilde{T}_{l m}^{j} \bar{q}^{m}+\delta_{k}^{j} \widetilde{T}_{l m}^{i} \bar{q}^{m}\right)\right\} \\
& +c_{3}\left\{\frac{1}{2 \sqrt{2}}\left(S_{k l}^{i} \bar{q}^{j}+S_{k l}^{j} \bar{q}^{i}\right)-\frac{1}{10 \sqrt{30}}\left(\delta_{l}^{i} S_{k m}^{j} \bar{q}^{m}+\delta_{l}^{j} S_{k m}^{i} \bar{q}^{m}+\delta_{k}^{i} S_{l m}^{j} \bar{q}^{m}+\delta_{k}^{j} S_{l m}^{i} \bar{q}^{m}\right)\right\}, \\
T_{i j k l}^{a}= & T_{i j k l} \bar{q}^{a}-\frac{1}{6}\left(\delta_{i}^{a} T_{j k l m} \bar{q}^{m}+\delta_{j}^{a} T_{i k l m} \bar{q}^{m}+\delta_{k}^{a} T_{i j l m} \bar{q}^{m}+\delta_{l}^{a} T_{i j k m} \bar{q}^{m}\right),
\end{aligned}
$$

where $S^{i j}, T_{i}, Q_{i}, T_{j k}^{i}, \widetilde{Q}_{i}, \widetilde{T}_{j k}^{i}, T^{i j}, S_{j k}^{i}$, and $T_{i j k l}$ are defined in Eqs. (2.10), (2.12), (2.14), and (2.16). This is consistent with the fact that

$$
\mathbf{3} \otimes \mathbf{3} \otimes \mathbf{3} \otimes \mathbf{3} \otimes \overline{\mathbf{3}}=\mathbf{3 5} \oplus(3) \mathbf{2 7} \oplus(2) \overline{\mathbf{1 0}} \oplus(4) \mathbf{1 0} \oplus(8) \mathbf{8} \oplus(3) \mathbf{1},
$$

where the numbers in parentheses are the number of multiplicity. Here, we do not give the full list of the pentaquark wave function explicitly in terms of $u, d$, and $s$ quarks, since they can be read directly from the results presented in this Section. The coefficients $c_{i}$ are to be determined by quark dynamics. For example, in the case of antidecuplet, Jaffe-Wilczek model favors $\overline{\mathbf{3}}$ structure of a diquark, hence $c_{2}=0[60,62]$, while $c_{2} \neq 0$ in SU(3) quark model of Carlson et al. [88]. Furthermore, if the diquark inside pentaquark baryons cannot form $\mathbf{6}$, the pentaquark 10, 27, and $\mathbf{3 5}$ are hard to be observed in the diquark-diquark-antiquark model. Therefore, observation of other pentaquark states in higher multiplets will help us to understand the structure of pentaquark baryons.

\section{INTERACTIONS OF PENTAQUARK BARYONS}

With the wave functions of pentaquark baryons constructed in the previous Section, we now consider the SU(3) symmetric interaction Lagrangian of pentaquark baryons. In this paper, we first consider pentaquark interactions with three-quark baryons and meson octet. Then we establish the pentaquark interactions with (other) pentaquarks and meson octet. Some of the results presented in this Section can be read from the tables of Ref. [94], which, however, does not give all relations for pentaquark-pentaquark interactions. Since we are interested in the flavor $\mathrm{SU}(3)$ symmetric structure of the interactions and the spin-parity of pentaquark baryons is yet to be determined, we drop the Lorenz structure in this study.

\section{A. Interactions with 3-quark octet and meson octet}

We first consider the pentaquark interactions with normal baryon octet $\left(\mathbf{8}_{3}\right)$ and meson octet $\left(\mathbf{8}_{M}\right)$. In this case, we note that

$$
\mathbf{8} \otimes \mathbf{8}=\mathbf{2 7} \oplus \mathbf{1 0} \oplus \overline{\mathbf{1 0}} \oplus \mathbf{8}_{1} \oplus \mathbf{8}_{2} \oplus \mathbf{1}
$$


The SU(3)-invariant interactions can be obtained by constructing SU(3) singlet. In tensor notation, it is achieved by fully contracting the upper and lower indices of the three tensors representing two baryon multiplets and meson octet. When the number of upper indices does not match that of lower indices, the Levi-Civita tensors $\epsilon_{i j k}$ are introduced to make the interactions fully contracted. In this construction, 35-8-8 cannot be SU(3)-invariant and the 35-plet cannot couple to 3 -quark octet and meson octet. This property of the 35-plet would be useful to identify the 35-plet members in experiments.

The 3-quark baryon octet and meson octet read

$$
\begin{aligned}
& B_{1}^{3}=p, \quad B_{2}^{3}=n, \quad B_{1}^{2}=\Sigma^{+}, \\
& B_{2}^{1}=\Sigma^{-}, \quad B_{1}^{1}=1 / \sqrt{2} \Sigma^{0}+1 / \sqrt{6} \Lambda^{0}, \quad B_{2}^{2}=-1 / \sqrt{2} \Sigma^{0}+1 / \sqrt{6} \Lambda^{0}, \\
& B_{3}^{3}=-\sqrt{2 / 3} \Lambda^{0}, \quad B_{3}^{2}=\Xi^{0}, \quad B_{3}^{1}=-\Xi^{-},
\end{aligned}
$$

and

$$
\begin{aligned}
& M_{1}^{3}=K^{+}, \quad M_{2}^{3}=K^{0}, \quad M_{1}^{2}=\pi^{+}, \\
& M_{2}^{1}=\pi^{-}, \quad M_{1}^{1}=1 / \sqrt{2} \pi^{0}+1 / \sqrt{6} \eta, \quad M_{2}^{2}=-1 / \sqrt{2} \pi^{0}+1 / \sqrt{6} \eta, \\
& M_{3}^{3}=-\sqrt{2 / 3} \eta, \quad M_{3}^{2}=\bar{K}^{0}, \quad M_{3}^{1}=K^{-} .
\end{aligned}
$$

It is also useful to define isospin multiplets as

$$
\begin{aligned}
& N=\left(\begin{array}{c}
p \\
n
\end{array}\right), \quad \Xi=\left(\begin{array}{c}
\Xi^{0} \\
\Xi^{-}
\end{array}\right), \quad K=\left(\begin{array}{c}
K^{+} \\
K^{0}
\end{array}\right), \quad K_{c}=\left(\begin{array}{c}
\bar{K}^{0} \\
-K^{-}
\end{array}\right), \\
& \bar{K}=\left(K^{-}, \bar{K}^{0}\right), \quad \bar{K}_{c}=\left(K^{0}, \quad-K^{+}\right), \quad \Sigma=\left(\Sigma^{+}, \Sigma^{0}, \Sigma^{-}\right)^{T}, \\
& \boldsymbol{\tau} \cdot \boldsymbol{\Sigma}=\left(\begin{array}{cc}
\Sigma^{0} & \sqrt{2} \Sigma^{+} \\
\sqrt{2} \Sigma^{-} & -\Sigma^{0}
\end{array}\right), \quad \boldsymbol{\tau} \cdot \boldsymbol{\pi}=\left(\begin{array}{cc}
\pi^{0} & \sqrt{2} \pi^{+} \\
\sqrt{2} \pi^{-} & -\pi^{0}
\end{array}\right) .
\end{aligned}
$$

We now construct the SU(3)-invariant Lagrangian for pentaquark baryons up to the universal coupling constants. The pentaquark baryon fields, $S, P_{i}^{j}, D_{i j k}, T^{i j k}, T_{i j}^{k l}$, and $T_{i j k l}^{a}$ are defined in Section III.

\section{1. $1-8_{3}$}

We start by considering the interaction of pentaquark singlet with (three-quark) baryon octet and meson octet. There is only one possible way to contract the upper and lower indices in this case and the $\mathrm{SU}(3)$ invariant Lagrangian reads

$$
\mathcal{L}_{1-8_{3}}=g_{1-8_{3}} \bar{S} B_{k}^{i} M_{i}^{k}+\text { (H.c.). }
$$

Using Eqs.(3.5), (4.2), and (4.3), we obtain

$$
\mathcal{L}_{\mathbf{1 - 8 _ { 3 }}} / g_{1-\mathbf{8}_{3}}=-\bar{\Lambda}_{1}^{0}\left(\bar{K} N+\bar{K}_{c} \Xi+\boldsymbol{\Sigma} \cdot \boldsymbol{\pi}+\Lambda \eta\right)+(\text { H.c. }),
$$

where

$$
\boldsymbol{\Sigma} \cdot \boldsymbol{\pi}=\Sigma^{+} \pi^{-}+\Sigma^{0} \pi^{0}+\Sigma^{-} \pi^{+},
$$

and the isospin multiplets are defined in Eq. (4.4).

\section{2. $8-8_{3}$}

For the interaction of pentaquark octet and normal baryon octet and meson octet, there are two possible ways to contract the upper and lower indices. This is equivalent to the well-known $f$-and $d$-type interactions and the interaction Lagrangian reads

$$
\mathcal{L}_{\mathbf{8 - \mathbf { 8 } _ { 3 }}} / g_{\mathbf{8}_{-\mathbf{8}_{3}}}=(d+f) \bar{P}_{i}^{l} B_{k}^{i} M_{l}^{k}+(d-f) \bar{P}_{i}^{l} B_{l}^{k} M_{k}^{i}+(\text { H.c. }) .
$$


Using identification of the pentaquark octet given in Sect. III and Eqs. (4.2) and (4.3), we obtain

$$
\begin{aligned}
\mathcal{L}_{\mathbf{8 - \mathbf { 8 } _ { 3 }}} / g_{\mathbf{8}-\mathbf{8}_{3}}= & \frac{1}{\sqrt{2}}(d+f) \bar{N}_{8} \boldsymbol{\tau} \cdot \boldsymbol{\pi} N-\frac{1}{\sqrt{6}}(d-3 f) \bar{N}_{8} N \eta-\frac{1}{\sqrt{6}}(d+3 f) \bar{N}_{8} K \Lambda^{0} \\
& +\frac{1}{\sqrt{2}}(d-f) \bar{N}_{8} \boldsymbol{\tau} \cdot \boldsymbol{\Sigma} K-i \sqrt{2} f\left(\overline{\boldsymbol{\Sigma}}_{8} \times \boldsymbol{\Sigma}\right) \cdot \boldsymbol{\pi}+\sqrt{\frac{2}{3}} d \bar{\Sigma}_{8} \Sigma \eta \\
& +\sqrt{\frac{2}{3}} d \overline{\boldsymbol{\Sigma}}_{8} \cdot \boldsymbol{\pi} \Lambda^{0}-\frac{1}{\sqrt{2}}(d+f) \bar{K}_{c} \overline{\boldsymbol{\Sigma}}_{8} \cdot \boldsymbol{\tau} \Xi+\frac{1}{\sqrt{2}}(d-f) \bar{K} \overline{\boldsymbol{\Sigma}}_{8} \cdot \boldsymbol{\tau} N \\
& +\sqrt{\frac{2}{3}} d \bar{\Lambda}_{8}^{0} \boldsymbol{\Sigma} \cdot \boldsymbol{\pi}-\sqrt{\frac{2}{3}} d \bar{\Lambda}_{8}^{0} \Lambda^{0} \eta-\frac{1}{\sqrt{6}}(d-3 f) \bar{\Lambda}_{8}^{0} \bar{K}_{c} \Xi \\
& -\frac{1}{\sqrt{6}}(d+3 f) \bar{\Lambda}_{8}^{0} \bar{K} N-\frac{1}{\sqrt{2}}(d-f) \bar{\Xi}_{8} \boldsymbol{\tau} \cdot \boldsymbol{\pi} \Xi \\
& -\frac{1}{\sqrt{6}}(d+3 f) \bar{\Xi}_{8} \Xi \eta-\frac{1}{\sqrt{2}}(d+f) \bar{\Xi}_{8} \boldsymbol{\tau} \cdot \boldsymbol{\Sigma} K_{c}-\frac{1}{\sqrt{6}}(d-3 f) \bar{\Xi}_{8} K_{c} \Lambda^{0} \\
& +(\text { H.c. }),
\end{aligned}
$$

where the isospin multiplets of $\boldsymbol{8}_{3}$ are defined as in Eq. (4.4) and

$$
\begin{aligned}
-i\left(\overline{\boldsymbol{\Sigma}}_{8} \times \boldsymbol{\Sigma}\right) \cdot \boldsymbol{\pi} & =\bar{\Sigma}_{8}^{+}\left(\Sigma^{+} \pi^{0}-\Sigma^{0} \pi^{+}\right)+\bar{\Sigma}_{8}^{0}\left(\Sigma^{-} \pi^{+}-\Sigma^{+} \pi^{-}\right)+\bar{\Sigma}_{8}^{-}\left(\Sigma^{0} \pi^{-}-\Sigma^{-} \pi^{0}\right), \\
\overline{\boldsymbol{\Sigma}}_{8} \cdot \boldsymbol{\Sigma} & =\bar{\Sigma}_{8}^{+} \Sigma^{+}+\bar{\Sigma}_{8}^{0} \Sigma^{0}+\bar{\Sigma}_{8}^{-} \Sigma^{-} .
\end{aligned}
$$

In Refs. [62, 63], it has been claimed that the OZI rule leads to $f / d=1 / 3$ within the ideal mixing of pentaquark octet and antidecuplet.

\section{3. $10-\mathbf{8}_{3}$}

The SU(3) interaction Lagrangian of pentaquark decuplet with the normal baryon octet and meson octet can be constructed as

$$
\mathcal{L}_{\mathbf{1 0 - \mathbf { 8 } _ { 3 }}}=g_{\mathbf{1 0 - \mathbf { 8 } _ { 3 }}} \epsilon_{i j k} \bar{D}^{j l m} B_{l}^{i} M_{m}^{k}+\text { (H.c.). }
$$

Since the number of the upper indices is not the same with that of the lower indices, $\epsilon_{i j k}$ has been introduced to form the fully contracted interaction. Other possible contractions are equivalent to Eq. (4.11) up to an overall sign.

Noting that the indices of the pentaquark decuplet are symmetric, we work out all the contractions and find

$$
\begin{aligned}
& \mathcal{L}_{\mathbf{1 0 - 8 _ { 3 }}} / g_{\mathbf{1 0 - 8 _ { 3 }}}=-\sqrt{3 \bar{\Delta}_{10}} \boldsymbol{T}\left(\frac{3}{2}, \frac{1}{2}\right) \cdot \boldsymbol{\pi} N+{\sqrt{3 \Delta_{10}}} \boldsymbol{T}\left(\frac{3}{2}, \frac{1}{2}\right) \cdot \boldsymbol{\Sigma} K+\bar{K} \overline{\boldsymbol{\Sigma}}_{10} \cdot \boldsymbol{\tau} N \\
& -i\left(\overline{\boldsymbol{\Sigma}}_{10} \times \boldsymbol{\Sigma}\right) \cdot \boldsymbol{\pi}+\sqrt{3} \bar{\Sigma}_{10} \Sigma \eta-\sqrt{3} \overline{\boldsymbol{\Sigma}}_{10} \cdot \boldsymbol{\pi} \Lambda^{0} \\
& +\bar{K}_{c} \overline{\boldsymbol{\Sigma}}_{10} \cdot \boldsymbol{\tau} \Xi-\bar{\Xi}_{10} \boldsymbol{\tau} \cdot \boldsymbol{\Sigma} K_{c}+\bar{\Xi}_{10} \boldsymbol{\tau} \cdot \boldsymbol{\pi} \Xi \\
& +\sqrt{3} \bar{\Xi}_{10} \Xi \eta-\sqrt{3} \bar{\Xi}_{10} K_{c} \Lambda^{0}-\sqrt{6} \bar{\Omega}_{10}^{-} \bar{K} \Xi+\text { (H.c.), }
\end{aligned}
$$

where

$$
\Delta_{10}=\left(\Delta_{10}^{++}, \Delta_{10}^{+}, \Delta_{10}^{0}, \Delta_{10}^{-}\right)^{T} .
$$

The other isospin multiplets are defined as in Eq. (4.4) and

$$
\boldsymbol{T}\left(\frac{3}{2}, \frac{1}{2}\right) \cdot \boldsymbol{\pi}=-T_{3 / 2,1 / 2}^{(+1)} \pi^{+}+T_{3 / 2,1 / 2}^{(-1)} \pi^{-}+T_{3 / 2,1 / 2}^{(0)} \pi^{0},
$$

with

$$
T_{\frac{3}{2}, \frac{1}{2}}^{(+1)}=\frac{1}{\sqrt{3}}\left(\begin{array}{cc}
\sqrt{6} & 0 \\
0 & \sqrt{2} \\
0 & 0 \\
0 & 0
\end{array}\right), \quad T_{\frac{3}{2}, \frac{1}{2}}^{(0)}=\frac{1}{\sqrt{3}}\left(\begin{array}{cc}
0 & 0 \\
2 & 0 \\
0 & 2 \\
0 & 0
\end{array}\right), \quad T_{\frac{3}{2}, \frac{1}{2}}^{(-1)}=\frac{1}{\sqrt{3}}\left(\begin{array}{cc}
0 & 0 \\
0 & 0 \\
\sqrt{2} & 0 \\
0 & \sqrt{6}
\end{array}\right)
$$


Therefore, for example,

$$
\begin{aligned}
\bar{\Delta}_{10} \boldsymbol{T}\left(\frac{3}{2}, \frac{1}{2}\right) \cdot \boldsymbol{\pi} N= & -\sqrt{2} \bar{\Delta}_{10}^{++} N^{+} \pi^{+}-\sqrt{\frac{2}{3}} \bar{\Delta}_{10}^{+}\left(N^{0} \pi^{+}-\sqrt{2} N^{+} \pi^{0}\right) \\
& +\sqrt{\frac{2}{3}} \bar{\Delta}_{10}^{0}\left(N^{+} \pi^{-}+\sqrt{2} N^{0} \pi^{0}\right)+\sqrt{2} \bar{\Delta}_{10}^{-} N^{0} \pi^{-} .
\end{aligned}
$$

In a similar way, one can calculate $\bar{\Delta}_{10} \boldsymbol{T}\left(\frac{3}{2}, \frac{1}{2}\right) \cdot \boldsymbol{\Sigma} K$. The generalized isospin matrices are defined as [98]

$$
T_{\nu \mu}^{(\rho)}\left(I^{\prime}, I\right)=\sqrt{\frac{\left(2 I^{\prime}+1\right)(2 I+1)}{3}}(-1)^{I-\mu}\left\langle I^{\prime} \nu I-\mu \mid 1 \rho\right\rangle
$$

in the spherical basis.

\section{4. $\overline{10}-8_{3}$}

The SU(3) symmetric Lagrangian for this interaction was reported in Ref. [61], which reads

$$
\mathcal{L}_{\overline{\mathbf{1 0}}-\mathbf{8}_{3}}=g_{\overline{\mathbf{1 0}}-\mathbf{8}_{3}} \epsilon^{i l m} \bar{T}_{i j k} B_{l}^{j} M_{m}^{k}+(\text { H.c. }) .
$$

Then we have [63]

$$
\begin{aligned}
\mathcal{L}_{\overline{\mathbf{1 0}}-\mathbf{8}_{3}} / g_{\overline{\mathbf{1 0}}-\mathbf{8}_{3}}= & -\sqrt{6} \bar{\Theta}^{+} \bar{K}_{c} N-\bar{N}_{\overline{10}} \boldsymbol{\tau} \cdot \boldsymbol{\pi} N+\sqrt{3} \bar{N}_{\overline{10}} N \eta \\
& -\sqrt{3} \bar{N}_{\overline{10}} K \Lambda^{0}+\bar{N}_{\overline{10}} \boldsymbol{\tau} \cdot \boldsymbol{\Sigma} K+i\left(\overline{\boldsymbol{\Sigma}}_{\overline{10}} \times \boldsymbol{\Sigma}\right) \cdot \boldsymbol{\pi} \\
& -\sqrt{3} \overline{\boldsymbol{\Sigma}}_{\overline{10}} \cdot \boldsymbol{\pi} \Lambda^{0}+\sqrt{3} \bar{\Sigma}_{\overline{10}} \Sigma \eta-\bar{K}_{c} \overline{\boldsymbol{\Sigma}}_{\overline{10}} \cdot \boldsymbol{\tau} \Xi \\
& -\bar{K} \overline{\boldsymbol{\Sigma}}_{\overline{10}} \cdot \boldsymbol{\tau} N-\sqrt{3} \bar{\Xi}_{\overline{10}} \boldsymbol{T}\left(\frac{3}{2}, \frac{1}{2}\right) \cdot \boldsymbol{\pi} \Xi+\sqrt{3} \bar{\Xi}_{\overline{10}} \boldsymbol{T}\left(\frac{3}{2}, \frac{1}{2}\right) \cdot \boldsymbol{\Sigma} K_{c} \\
& +(\text { H.c. }),
\end{aligned}
$$

where

$$
\Xi_{\overline{10}}=\left(\Xi_{\overline{10}}^{+}, \Xi_{\overline{10}}^{0}, \Xi_{\overline{10}}^{-}, \Xi_{\overline{10}}^{--}\right)^{T}
$$

Note that

$$
\bar{\Theta}^{+} \bar{K}_{c} N=\bar{\Theta}^{+}\left(p K^{0}-n K^{+}\right),
$$

so that it has a relative negative sign for the $\Theta^{+}$decays into proton and neutron.

The universal coupling constant can be estimated from the decay width of $\Theta^{+}(1540)$ as

$$
g_{\frac{\mathbf{1 0}-\mathbf{8}_{3}}{2}}^{2}=\frac{\pi M_{\Theta} \Gamma_{\Theta}}{3\left|\boldsymbol{p}_{K}\right|\left(\sqrt{M_{N}^{2}+\boldsymbol{p}_{K}^{2}} \mp M_{N}\right)}
$$

where $M_{\Theta}$ and $\Gamma_{\Theta}$ are the mass and decay width of $\Theta^{+}(1540)$, respectively, and $p_{K}$ is the momentum of kaon in the $\Theta^{+}$rest frame. The upper and lower sign correspond to the case when the parity of $\Theta^{+}$is even and odd. Numerically, we have $g_{\overline{\mathbf{1 0}}-\mathbf{8}_{3}}^{2}=\Gamma_{\Theta} /(6.19 \mathrm{MeV})$ for the case that $\Theta(1540)$ has even-parity, which gives $g_{\overline{\mathbf{1 0}}-\mathbf{8}_{3}}=0.4$ if $\Gamma_{\Theta}=1 \mathrm{MeV}$ is used, and 0.9 if $\Gamma_{\Theta}=5 \mathrm{MeV}$. If $\Theta(1540)$ has odd-parity, then we have $g_{\overline{\mathbf{1 0}}-\mathbf{8}_{3}}^{2}=\Gamma_{\Theta} /(318.2 \mathrm{MeV})$, which gives $g_{\overline{\mathbf{1 0}}-\mathbf{8}_{3}}=0.056$ and 0.125 for $\Gamma_{\Theta}=1 \mathrm{MeV}$ and $5 \mathrm{MeV}$, respectively. We refer to Refs. [99-101] for detailed discussions on the decay width of $\Theta^{+}(1540)$.

\section{5. $27-8_{3}$}

In this case, the interaction can be written as

$$
\left.\mathcal{L}_{\mathbf{2 7 - \mathbf { 8 } _ { 3 }}}=g_{\mathbf{2 7 - \mathbf { 8 } _ { 3 }}} \bar{T}_{i j}^{k l} B_{k}^{i} M_{l}^{j}+\text { (H.c. }\right)
$$


Again, because of symmetric upper and lower indices of $\bar{T}_{i j}^{k l}$, other possible contractions are not independent from the above expression. In terms of the physical baryon states, we have

$$
\begin{aligned}
\mathcal{L}_{\mathbf{2 7 - \mathbf { 8 } _ { 3 }}} / g_{\mathbf{2 7 - 8}}= & \sqrt{2} \bar{K}_{c} \overline{\boldsymbol{\Theta}}_{1} \cdot \boldsymbol{\tau} N+\bar{\Delta}_{27} \boldsymbol{T}\left(\frac{3}{2}, \frac{1}{2}\right) \cdot \boldsymbol{\pi} N+\bar{\Delta}_{27} \boldsymbol{T}\left(\frac{3}{2}, \frac{1}{2}\right) \cdot \boldsymbol{\Sigma} K \\
& +\frac{1}{\sqrt{15}} \bar{N}_{27} \boldsymbol{\tau} \cdot \boldsymbol{\pi} N+\frac{1}{\sqrt{15}} \bar{N}_{27} \boldsymbol{\tau} \cdot \boldsymbol{\Sigma} K+\frac{3}{\sqrt{5}} \bar{N}_{27} K \Lambda^{0} \\
& +\frac{3}{\sqrt{5}} \bar{N}_{27} N \eta+\frac{2}{\sqrt{3}} \bar{\Sigma}_{27,2} \boldsymbol{T}(2,1) \cdot \boldsymbol{\pi} \Sigma_{c}-\sqrt{\frac{2}{5}} \bar{K}_{\boldsymbol{\Sigma}_{27} \cdot \boldsymbol{\tau} N} \\
& +\sqrt{\frac{2}{5}} \bar{K}_{c} \overline{\boldsymbol{\Sigma}}_{27} \cdot \boldsymbol{\tau} \Xi+\sqrt{\frac{6}{5}} \bar{\Sigma}_{27} \Sigma \eta+\sqrt{\frac{6}{5}} \overline{\boldsymbol{\Sigma}}_{27} \cdot \boldsymbol{\pi} \Lambda^{0} \\
& -\sqrt{\frac{3}{10}} \bar{\Lambda}_{27}^{0} \bar{K} N-\sqrt{\frac{3}{10}} \bar{\Lambda}_{27}^{0} \bar{K}_{c} \Xi+\frac{1}{\sqrt{30}} \bar{\Lambda}_{27}^{0} \boldsymbol{\Sigma} \cdot \boldsymbol{\pi} \\
& +3 \sqrt{\frac{3}{10}} \bar{\Lambda}_{27}^{0} \Lambda^{0} \eta+\bar{\Xi}_{27,3 / 2} \boldsymbol{T}\left(\frac{3}{2}, \frac{1}{2}\right) \cdot \boldsymbol{\Sigma} K_{c}+\bar{\Xi}_{27,3 / 2} \boldsymbol{T}\left(\frac{3}{2}, \frac{1}{2}\right) \cdot \boldsymbol{\pi} \Xi \\
& -\frac{1}{\sqrt{15}} \bar{\Xi}_{27} \boldsymbol{\tau} \cdot \boldsymbol{\Sigma} K_{c}-\frac{1}{\sqrt{15}} \bar{\Xi}_{27} \boldsymbol{\tau} \cdot \boldsymbol{\pi} \Xi+\frac{3}{\sqrt{5}} \bar{\Xi}_{27} K_{c} \Lambda^{0} \\
& +\frac{3}{\sqrt{5}} \bar{\Xi}_{27} \Xi \eta-\sqrt{2} \bar{K} \overline{\boldsymbol{\Omega}}_{27,1} \cdot \boldsymbol{\tau} \Xi+(\text { H.c. }),
\end{aligned}
$$

where

$$
\begin{gathered}
\Sigma_{c}=\left(-\Sigma^{+}, \Sigma^{0}, \Sigma^{-}\right)^{T}, \quad \Xi_{27,3 / 2}=\left(\Xi_{27,3 / 2}^{+}, \Xi_{27,3 / 2}^{0}, \Xi_{27,3 / 2}^{-}, \Xi_{27,3 / 2}^{--}\right)^{T}, \\
\boldsymbol{\tau} \cdot \boldsymbol{\Theta}_{1}=\left(\begin{array}{cc}
\Theta_{1}^{+} & \sqrt{2} \Theta_{1}^{++} \\
\sqrt{2} \Theta_{1}^{0} & -\Theta_{1}^{+}
\end{array}\right), \quad \boldsymbol{\tau} \cdot \boldsymbol{\Omega}_{27,1}=\left(\begin{array}{cc}
\Omega_{27,1}^{-} & \sqrt{2} \Omega_{27,1}^{0} \\
\sqrt{2} \Omega_{27,1}^{--} & -\Omega_{27,1}^{-}
\end{array}\right),
\end{gathered}
$$

and

$$
\boldsymbol{T}(2,1) \cdot \boldsymbol{\pi}=-T_{2,1}^{(+1)} \pi^{+}+T_{2,1}^{(-1)} \pi^{-}+T_{2,1}^{(0)} \pi^{0}
$$

with

$$
\begin{aligned}
& T_{2,1}^{(+1)}=\frac{1}{\sqrt{2}}\left(\begin{array}{ccc}
\sqrt{6} & 0 & 0 \\
0 & \sqrt{3} & 0 \\
0 & 0 & 1 \\
0 & 0 & 0 \\
0 & 0 & 0
\end{array}\right), T_{2,1}^{(0)}=\frac{1}{\sqrt{2}}\left(\begin{array}{ccc}
0 & 0 & 0 \\
\sqrt{3} & 0 & 0 \\
0 & 2 & 0 \\
0 & 0 & \sqrt{3} \\
0 & 0 & 0
\end{array}\right), \\
& T_{2,1}^{(-1)}=\frac{1}{\sqrt{2}}\left(\begin{array}{ccc}
0 & 0 & 0 \\
0 & 0 & 0 \\
1 & 0 & 0 \\
0 & \sqrt{3} & 0 \\
0 & 0 & \sqrt{6}
\end{array}\right) .
\end{aligned}
$$

Thus,

$$
\begin{aligned}
\bar{\Sigma}_{27,2} \boldsymbol{T}(2,1) \cdot \boldsymbol{\pi} \Sigma_{c}= & \sqrt{3} \bar{\Sigma}_{27,2}^{++} \Sigma^{+} \pi^{+}-\sqrt{\frac{3}{2}} \bar{\Sigma}_{27,2}^{+}\left(\Sigma^{0} \pi^{+}+\sqrt{\frac{3}{2}} \Sigma^{+} \pi^{0}\right)-\frac{1}{\sqrt{2}} \bar{\Sigma}_{27,2}^{0}\left(\Sigma^{-} \pi^{+}-2 \Sigma^{0} \pi^{0}+\Sigma^{+} \pi^{-}\right) \\
& +\sqrt{\frac{3}{2}} \bar{\Sigma}_{27,2}^{-}\left(\Sigma^{-} \pi^{0}+\Sigma^{0} \pi^{-}\right)+\sqrt{3} \bar{\Sigma}_{27,2}^{--} \Sigma^{-} \pi^{-}
\end{aligned}
$$

and

$$
\bar{K}_{c} \bar{\Theta}_{1} \cdot \boldsymbol{\tau} N=-\sqrt{2} \bar{\Theta}_{1}^{++} p K^{+}+\bar{\Theta}_{1}^{+}\left(K^{0} p+K^{+} n\right)+\sqrt{2} \bar{\Theta}_{1}^{0} n K^{0} .
$$

Note that $\Theta_{1}^{+}$decays into $K N$ quite similarly as the anti-decuplet $\Theta^{+}$does except the relative sign difference between the proton and neutron channel. This would make it difficult to disentangle the two if their mass difference is not big enough. Thus, the decay of $\Theta_{1}^{++}$and $\Theta_{1}^{0}$ would be useful to identify the $\Theta_{1}$ isomultiplet in $K N$ channel. 


\section{B. Interactions with 3-quark decuplet and meson octet}

We now move to interactions for pentaquarks with 3-quark decuplet and meson octet. In this case, we have

$$
10 \otimes 8=35 \oplus \mathbf{2 7} \oplus \mathbf{1 0} \oplus \mathbf{8}
$$

so that pentaquark antidecuplet cannot couple to this channel. Therefore this can give a useful constraint in finding antidecuplet baryons [61]. For example, N(1710) cannot be a pure antidecuplet state as we discussed before. As in the case of pentaquark decuplet, the three-quark decuplet is represented by $D_{i j k}^{(3)}$ as

$$
\begin{array}{lrlrl}
D_{111}^{(3)}=\sqrt{6} \Delta^{++}, & D_{112}^{(3)}=\sqrt{2} \Delta^{+}, & D_{122}^{(3)}=\sqrt{2} \Delta^{0}, \\
D_{222}^{(3)}=\sqrt{6} \Delta^{-}, & D_{113}^{(3)}=\sqrt{2} \Sigma^{*+}, & D_{123}^{(3)}=-\Sigma^{* 0}, \\
D_{223}^{(3)}=-\sqrt{2} \Sigma^{*-}, & D_{133}^{(3)}=\sqrt{2} \Xi^{* 0}, & D_{233}^{(3)}=\sqrt{2} \Xi^{*-}, \\
D_{333}^{(3)}=-\sqrt{6} \Omega^{-} . & &
\end{array}
$$

\section{1. $\mathbf{8 - 1 0} 0_{3}$}

The interaction Lagrangian for pentaquark octet and normal baryon decuplet reads

$$
\left.\mathcal{L}_{\mathbf{8 - 1 0}}=g_{\mathbf{8}_{-10} \mathbf{1 0}_{3}} \epsilon^{i j k} \bar{P}_{i}^{l} D_{j l m}^{(3)} M_{k}^{m}+\text { (H.c. }\right),
$$

which gives

$$
\begin{aligned}
\mathcal{L}_{\mathbf{8 - 1 0}} / g_{\mathbf{8 - 1 0}}= & \sqrt{3} \bar{N}_{8} \boldsymbol{T}\left(\frac{1}{2}, \frac{3}{2}\right) \cdot \boldsymbol{\pi} \Delta+\bar{N}_{8} \boldsymbol{\tau} \cdot \boldsymbol{\Sigma}^{*} K-i\left(\overline{\boldsymbol{\Sigma}}_{8} \times \boldsymbol{\Sigma}^{*}\right) \cdot \boldsymbol{\pi} \\
& +\sqrt{3} \bar{\Sigma}_{8} \Sigma^{*} \eta-\bar{K}_{c} \overline{\boldsymbol{\Sigma}}_{8} \cdot \boldsymbol{\tau} \Xi^{*}-\sqrt{3} \bar{K} \overline{\boldsymbol{\Sigma}}_{8} \cdot \boldsymbol{T}\left(\frac{1}{2}, \frac{3}{2}\right) \Delta \\
& -\sqrt{3} \bar{\Lambda}_{8}^{0} \boldsymbol{\Sigma}^{*} \cdot \boldsymbol{\pi}-\sqrt{3} \bar{\Lambda}_{8}^{0} \bar{K}_{c} \Xi^{*}+\bar{\Xi}_{8} \boldsymbol{\tau} \cdot \boldsymbol{\pi} \Xi^{*} \\
& +\sqrt{3} \bar{\Xi}_{8} \Xi^{*} \eta-\sqrt{6} \bar{\Xi}_{8} K \Omega^{-}+\bar{\Xi}_{8} \boldsymbol{\tau} \cdot \boldsymbol{\Sigma}^{*} K_{c}+\text { (H.c.), }
\end{aligned}
$$

where

$$
\boldsymbol{T}\left(\frac{1}{2}, \frac{3}{2}\right) \cdot \boldsymbol{\pi}=-T_{1 / 2,3 / 2}^{(+1)} \pi^{-}+T_{1 / 2,3 / 2}^{(-1)} \pi^{+}+T_{1 / 2,3 / 2}^{(0)} \pi^{0},
$$

and

$$
\begin{aligned}
& T_{1 / 2,3 / 2}^{(+1)}=-\frac{1}{\sqrt{3}}\left(\begin{array}{cccc}
\sqrt{6} & 0 & 0 & 0 \\
0 & \sqrt{2} & 0 & 0
\end{array}\right), \quad T_{1 / 2,3 / 2}^{(0)}=-\frac{1}{\sqrt{3}}\left(\begin{array}{llll}
0 & 2 & 0 & 0 \\
0 & 0 & 2 & 0
\end{array}\right), \\
& T_{1 / 2,3 / 2}^{(-1)}=-\frac{1}{\sqrt{3}}\left(\begin{array}{cccc}
0 & 0 & \sqrt{2} & 0 \\
0 & 0 & 0 & \sqrt{6}
\end{array}\right),
\end{aligned}
$$

so that

$$
\begin{aligned}
\bar{N}_{8} \boldsymbol{T}\left(\frac{1}{2}, \frac{3}{2}\right) \cdot \boldsymbol{\pi} \Delta= & \bar{N}_{8}^{+}\left(\sqrt{2} \Delta^{++} \pi^{-}-\sqrt{\frac{2}{3}} \Delta^{0} \pi^{+}-\frac{2}{\sqrt{3}} \Delta^{+} \pi^{0}\right) \\
& +\bar{N}_{8}^{0}\left(\sqrt{\frac{2}{3}} \Delta^{+} \pi^{-}-\sqrt{2} \Delta^{-} \pi^{+}-\frac{2}{\sqrt{3}} \Delta^{0} \pi^{0}\right) .
\end{aligned}
$$

Note that [98]

$$
\boldsymbol{T}\left(\frac{1}{2}, \frac{3}{2}\right)=-\boldsymbol{T}\left(\frac{3}{2}, \frac{1}{2}\right)^{\dagger},
$$

and the difference between Eqs. (4.14) and (4.34). 


\section{2. $\mathbf{1 0}-10_{3}$}

The interaction Lagrangian reads

$$
\mathcal{L}_{\mathbf{1 0 - 1 0}_{3}}=g_{\mathbf{1 0}_{-10}} \bar{D}^{j k l} D_{m k l}^{(3)} M_{j}^{m}+(\text { H.c. }),
$$

which leads to

$$
\begin{aligned}
\mathcal{L}_{\mathbf{1 0 - 1 0}_{3}} / g_{\mathbf{1 0 - 1 0}_{3}=} & \sqrt{\frac{15}{2}} \bar{\Delta}_{10} \boldsymbol{T}\left(\frac{3}{2}, \frac{3}{2}\right) \cdot \boldsymbol{\pi} \Delta+\sqrt{6} \bar{\Delta}_{10} \Delta \eta-\sqrt{6} \bar{\Delta}_{10} \boldsymbol{T}\left(\frac{3}{2}, \frac{1}{2}\right) \cdot \boldsymbol{\Sigma}^{*} K \\
& -i 2 \sqrt{2}\left(\overline{\boldsymbol{\Sigma}}_{10} \times \boldsymbol{\Sigma}^{*}\right) \cdot \boldsymbol{\pi}-2 \sqrt{2} \bar{K}_{c} \overline{\boldsymbol{\Sigma}}_{10} \cdot \boldsymbol{\tau} \Xi^{*}+\sqrt{6} \bar{K}_{\overline{\boldsymbol{\Sigma}}} \cdot \boldsymbol{T}\left(\frac{1}{2}, \frac{3}{2}\right) \Delta \\
& +\sqrt{2} \bar{\Xi}_{10} \boldsymbol{\tau} \cdot \boldsymbol{\pi} \Xi^{*}-\sqrt{6} \bar{\Xi}_{10} \Xi^{*} \eta-2 \sqrt{3} \bar{\Xi}_{10} K \Omega^{-} \\
& \left.-2 \sqrt{2} \bar{\Xi}_{10} \boldsymbol{\tau} \cdot \boldsymbol{\Sigma}^{*} K_{c}-2 \sqrt{6} \bar{\Omega}_{10}^{-} \Omega^{-} \eta-2 \sqrt{3} \bar{\Omega}_{10}^{-} \bar{K} \Xi^{*}+\text { (H.c. }\right),
\end{aligned}
$$

where

$$
\boldsymbol{T}\left(\frac{3}{2}, \frac{3}{2}\right) \cdot \boldsymbol{\pi}=-T_{3 / 2,3 / 2}^{(+1)} \pi^{+}+T_{3 / 2,3 / 2}^{(-1)} \pi^{-}+T_{3 / 2,3 / 2}^{(0)} \pi^{0}
$$

and

$$
\begin{aligned}
& T_{3 / 2,3 / 2}^{(+1)}=-\frac{4}{\sqrt{30}}\left(\begin{array}{cccc}
0 & \sqrt{3} & 0 & 0 \\
0 & 0 & 2 & 0 \\
0 & 0 & 0 & \sqrt{3} \\
0 & 0 & 0 & 0
\end{array}\right), T_{3 / 2,3 / 2}^{(0)}=\frac{2}{\sqrt{15}}\left(\begin{array}{cccc}
3 & 0 & 0 & 0 \\
0 & 1 & 0 & 0 \\
0 & 0 & -1 & 0 \\
0 & 0 & 0 & -3
\end{array}\right) \\
& T_{3 / 2,3 / 2}^{(-1)}=\frac{4}{\sqrt{30}}\left(\begin{array}{cccc}
0 & 0 & 0 & 0 \\
\sqrt{3} & 0 & 0 & 0 \\
0 & 2 & 0 & 0 \\
0 & 0 & \sqrt{3} & 0
\end{array}\right) .
\end{aligned}
$$

Thus we have

$$
\begin{aligned}
\bar{\Delta}_{10} \boldsymbol{T}\left(\frac{3}{2}, \frac{3}{2}\right) \cdot \boldsymbol{\pi} \Delta= & \sqrt{\frac{6}{5}} \bar{\Delta}_{10}^{++}\left(\sqrt{2} \Delta_{10}^{++} \pi^{0}+\frac{2}{\sqrt{3}} \Delta^{+} \pi^{+}\right)+\sqrt{\frac{6}{5}} \bar{\Delta}_{10}^{+}\left(\frac{2}{\sqrt{3}} \Delta^{++} \pi^{-}+\frac{\sqrt{2}}{3} \Delta^{+} \pi^{0}+\frac{4}{3} \Delta^{0} \pi^{+}\right) \\
& +\sqrt{\frac{6}{5}} \bar{\Delta}_{10}^{0}\left(\frac{4}{3} \Delta^{+} \pi^{-}-\frac{\sqrt{2}}{3} \Delta^{0} \pi^{0}+\frac{2}{\sqrt{3}} \Delta^{-} \pi^{+}\right)+\sqrt{\frac{6}{5}} \bar{\Delta}_{10}^{-}\left(\frac{2}{\sqrt{3}} \Delta^{0} \pi^{-}-\sqrt{2} \Delta^{-} \pi^{0}\right) .
\end{aligned}
$$

3. $\mathbf{2 7 - 1 0} 0_{3}$

The Lagrangian of this interaction reads

$$
\mathcal{L}_{\mathbf{2 7 - 1 0} \mathbf{1 0}_{3}}=g_{\mathbf{2 7 - 1 0} 3} \epsilon^{i m n} \bar{T}_{i j}^{k l} D_{m k l}^{(3)} M_{n}^{j}+(\text { H.c. }),
$$


which gives

$$
\begin{aligned}
\mathcal{L}_{\mathbf{2 7 - 1 0 _ { 3 }}} / g_{\mathbf{2 7 - 1 0} 3}= & -2 \sqrt{3} \bar{K}_{c} \overline{\boldsymbol{\Theta}}_{1} \cdot \boldsymbol{T}\left(\frac{1}{2}, \frac{3}{2}\right) \Delta+\sqrt{\frac{5}{2}} \bar{\Delta}_{27} \boldsymbol{T}\left(\frac{3}{2}, \frac{3}{2}\right) \cdot \boldsymbol{\pi} \Delta-3 \sqrt{2} \bar{\Delta}_{27} \Delta \eta \\
& +\sqrt{2} \overline{\boldsymbol{\Delta}}_{27} \boldsymbol{T}\left(\frac{3}{2}, \frac{1}{2}\right) \cdot \boldsymbol{\Sigma}^{*} K+\frac{2 \sqrt{2}}{\sqrt{5}} \bar{N}_{27} \boldsymbol{T}\left(\frac{1}{2}, \frac{3}{2}\right) \cdot \boldsymbol{\pi} \Delta \\
& -\frac{8 \sqrt{2}}{\sqrt{15}} \bar{N}_{27} \boldsymbol{\tau} \cdot \boldsymbol{\Sigma}^{*} K-3 \bar{\Sigma}_{27,2} \boldsymbol{I}\left(2, \frac{3}{2}\right) \cdot \boldsymbol{K}_{c} \Delta \\
& +\frac{2 \sqrt{2}}{\sqrt{3}} \bar{\Sigma}_{27,2} \boldsymbol{T}(2,1) \cdot \boldsymbol{\pi} \Sigma_{c}^{*}-i \frac{6}{\sqrt{5}}\left(\overline{\boldsymbol{\Sigma}}_{27} \times \boldsymbol{\Sigma}^{*}\right) \cdot \boldsymbol{\pi} \\
& +\frac{4}{\sqrt{5}} \bar{K}_{c} \overline{\boldsymbol{\Sigma}}_{27} \cdot \boldsymbol{\tau} \Xi^{*}-\frac{4 \sqrt{3}}{\sqrt{5}} \bar{\Sigma}_{27} \Sigma^{*} \eta-\sqrt{\frac{3}{5}} \bar{K}_{27} \cdot \boldsymbol{T}\left(\frac{1}{2}, \frac{3}{2}\right) \Delta \\
& -\frac{8}{\sqrt{15}} \bar{\Lambda}_{27}^{0} \boldsymbol{\Sigma}^{*} \cdot \boldsymbol{\pi}+\frac{4 \sqrt{15}}{5} \bar{\Lambda}_{27}^{0} \bar{K}_{c} \Xi^{*}+2 \sqrt{2} \bar{\Xi}_{27,3 / 2} \boldsymbol{T}\left(\frac{3}{2}, \frac{1}{2}\right) \cdot \boldsymbol{\pi} \Xi^{*} \\
& -2 \sqrt{2} \bar{\Xi}_{27,3 / 2} \boldsymbol{T}\left(\frac{3}{2}, \frac{1}{2}\right) \cdot \boldsymbol{\Sigma}^{*} K_{c}+\frac{7 \sqrt{2}}{\sqrt{15}} \bar{\Xi}_{27} \boldsymbol{\tau} \cdot \boldsymbol{\pi} \Xi^{*}-\frac{3 \sqrt{2}}{\sqrt{5}} \bar{\Xi}_{27} \Xi^{*} \eta \\
& +\frac{6}{\sqrt{5}} \bar{\Xi}_{27} K \Omega^{-}+\frac{2 \sqrt{2}}{\sqrt{15}} \bar{\Xi}_{27} \boldsymbol{\tau} \cdot \boldsymbol{\Sigma}^{*} K_{c}+2 \sqrt{6} \overline{\boldsymbol{\Omega}}_{27,1} \cdot \boldsymbol{\pi} \Omega^{-} \\
& +2 \bar{K} \overline{\boldsymbol{\Omega}}_{27,1} \cdot \boldsymbol{\tau} \Xi^{*}+(\mathrm{H.c.}),
\end{aligned}
$$

where

$$
\boldsymbol{I}\left(2, \frac{3}{2}\right) \cdot \boldsymbol{K}_{c}=I_{2,3 / 2}^{1 / 2} \bar{K}^{0}-I_{2,3 / 2}^{-1 / 2} K^{-},
$$

with

$$
I_{2,3 / 2}^{1 / 2}=\sqrt{\frac{2}{3}}\left(\begin{array}{cccc}
2 & 0 & 0 & 0 \\
0 & \sqrt{3} & 0 & 0 \\
0 & 0 & \sqrt{2} & 0 \\
0 & 0 & 0 & 1 \\
0 & 0 & 0 & 0
\end{array}\right), \quad I_{2,3 / 2}^{-1 / 2}=\sqrt{\frac{2}{3}}\left(\begin{array}{cccc}
0 & 0 & 0 & 0 \\
1 & 0 & 0 & 0 \\
0 & \sqrt{2} & 0 & 0 \\
0 & 0 & \sqrt{3} & 0 \\
0 & 0 & 0 & 2
\end{array}\right)
$$

So we have

$$
\begin{aligned}
\bar{K}_{c} \overline{\boldsymbol{\Theta}}_{1} \cdot \boldsymbol{T}\left(\frac{1}{2}, \frac{3}{2}\right) \Delta= & -\sqrt{\frac{2}{3}} \bar{\Theta}_{1}^{++}\left(\Delta^{+} K^{+}-\sqrt{3} \Delta^{++} K^{0}\right)+\frac{2}{\sqrt{3}} \bar{\Theta}_{1}^{+}\left(\Delta^{0} K^{+}-\Delta^{+} K^{0}\right) \\
& +\sqrt{\frac{2}{3}} \bar{\Theta}_{1}^{0}\left(\sqrt{3} \Delta^{-} K^{+}-\Delta^{0} K^{0}\right), \\
\bar{\Sigma}_{27,2} \boldsymbol{I}\left(2, \frac{3}{2}\right) \cdot \boldsymbol{K}_{c} \Delta= & \frac{2 \sqrt{2}}{\sqrt{3}} \bar{\Sigma}_{27,2}^{++} \Delta^{++} \bar{K}^{0}-\sqrt{\frac{2}{3}}\left(\Delta^{++} K^{-}-\sqrt{3} \Delta^{+} \bar{K}^{0}\right)-\frac{2}{\sqrt{3}}\left(\Delta^{+} K^{-}-\Delta^{0} \bar{K}^{0}\right) \\
& -\sqrt{\frac{2}{3}}\left(\sqrt{3} \Delta^{0} K^{-}-\Delta^{-} \bar{K}^{0}\right)-\frac{2 \sqrt{2}}{\sqrt{3}} \bar{\Sigma}_{27,2}^{--} \Delta^{-} K^{-} .
\end{aligned}
$$

As $\Theta_{1}$ can decay into $K \Delta$, this decay channel can distinguish $\Theta_{1}^{+}$from $\Theta^{+}$.

\section{4. $\quad 35-10_{3}$}

The interaction Lagrangian reads

$$
\mathcal{L}_{\mathbf{3 5}^{-10_{3}}}=g_{\mathbf{3 5 - 1 0} \mathbf{0}_{3}} \bar{T}_{a}^{i j k l} D_{i j k}^{(3)} M_{l}^{a}+\text { (H.c.) },
$$


so that we have

$$
\begin{aligned}
& \mathcal{L}_{\mathbf{3 5 - 1 0} \mathbf{1 0}_{3}} / g_{\mathbf{3 5 - 1 0}_{3}}=\frac{6 \sqrt{3}}{\sqrt{2}} \bar{\Theta}_{2} \boldsymbol{I}\left(2, \frac{3}{2}\right) \cdot \boldsymbol{K} \Delta+6 \bar{\Delta}_{5 / 2} \boldsymbol{T}\left(\frac{5}{2}, \frac{3}{2}\right) \cdot \boldsymbol{\pi} \Delta+\frac{3}{2} \bar{\Delta}_{35} \boldsymbol{T}\left(\frac{3}{2}, \frac{3}{2}\right) \cdot \boldsymbol{\pi} \Delta \\
& +3 \sqrt{5} \bar{\Delta}_{35} \Delta \eta+3 \sqrt{5} \bar{\Delta}_{35} \boldsymbol{T}\left(\frac{3}{2}, \frac{1}{2}\right) \cdot \boldsymbol{\Sigma}^{*} K+6 \bar{\Sigma}_{35,2} \boldsymbol{T}(2,1) \cdot \boldsymbol{\pi} \Sigma^{*} \\
& +\frac{3 \sqrt{3}}{\sqrt{2}} \bar{\Sigma}_{35,2} \boldsymbol{I}\left(2, \frac{3}{2}\right) \cdot \boldsymbol{K}_{c} \Delta-i \sqrt{6}\left(\overline{\boldsymbol{\Sigma}}_{35} \times \boldsymbol{\Sigma}^{*}\right) \cdot \boldsymbol{\pi}+6 \sqrt{2} \bar{\Sigma}_{35} \Sigma^{*} \eta \\
& +2 \sqrt{6} \bar{K}_{c} \overline{\boldsymbol{\Sigma}}_{35} \cdot \boldsymbol{\tau} \Xi^{*}+\frac{3}{\sqrt{2}} \bar{K} \overline{\boldsymbol{\Sigma}}_{35} \cdot \boldsymbol{T}\left(\frac{1}{2}, \frac{3}{2}\right) \Delta+6 \bar{\Xi}_{35,3 / 2} \boldsymbol{T}\left(\frac{3}{2}, \frac{1}{2}\right) \cdot \boldsymbol{\pi} \Xi^{*} \\
& +6 \bar{\Xi}_{35,3 / 2} \boldsymbol{T}\left(\frac{3}{2}, \frac{1}{2}\right) \cdot \boldsymbol{\Sigma}^{*} K_{c}+\sqrt{3} \bar{\Xi}_{35} \boldsymbol{\tau} \cdot \boldsymbol{\pi} \Xi^{*}+9 \bar{\Xi}_{35} \Xi \eta \\
& +3 \sqrt{2} \bar{\Xi}_{35} K \Omega^{-}-2 \sqrt{3} \bar{\Xi}_{35} \boldsymbol{\tau} \cdot \boldsymbol{\Sigma}^{*} K_{c}+6 \bar{\Omega}_{35,1} \cdot \boldsymbol{\pi} \Omega^{-} \\
& -3 \sqrt{6} \bar{K} \bar{\Omega}_{35,1} \cdot \tau \Xi^{*}+6 \sqrt{2} \bar{\Omega}_{35} \Omega^{-} \eta-6 \bar{\Omega}_{35}^{-} \bar{K}^{*} \\
& +12 \bar{X} K_{c} \Omega^{-}+\text {(H.c.), }
\end{aligned}
$$

where

$$
\begin{aligned}
\Delta_{5 / 2} & =\left(\Delta_{5 / 2}^{+++}, \Delta_{5 / 2}^{++}, \Delta_{5 / 2}^{+}, \Delta_{5 / 2}^{0}, \Delta_{5 / 2}^{-}, \Delta_{5 / 2}^{--}\right)^{T}, \\
\boldsymbol{T}\left(\frac{5}{2}, \frac{3}{2}\right) \cdot \boldsymbol{\pi} & =-T_{5 / 2,3 / 2}^{(+1)} \pi^{+}+T_{5 / 2,3 / 2}^{(-1)} \pi^{-}+T_{5 / 2,3 / 2}^{(0)} \pi^{0}
\end{aligned}
$$

and

$$
\begin{aligned}
& T_{5 / 2,3 / 2}^{(+1)}=\frac{1}{\sqrt{5}}\left(\begin{array}{cccc}
\sqrt{20} & 0 & 0 & 0 \\
0 & \sqrt{12} & 0 & 0 \\
0 & 0 & \sqrt{6} & 0 \\
0 & 0 & 0 & \sqrt{2} \\
0 & 0 & 0 & 0 \\
0 & 0 & 0 & 0
\end{array}\right), \quad T_{5 / 2,3 / 2}^{(0)}=\frac{2}{\sqrt{5}}\left(\begin{array}{cccc}
0 & 0 & 0 & 0 \\
\sqrt{2} & 0 & 0 & 0 \\
0 & \sqrt{3} & 0 & 0 \\
0 & 0 & \sqrt{3} & 0 \\
0 & 0 & 0 & \sqrt{2} \\
0 & 0 & 0 & 0
\end{array}\right), \\
& T_{5 / 2,3 / 2}^{(-1)}=\frac{1}{\sqrt{5}}\left(\begin{array}{cccc}
0 & 0 & 0 & 0 \\
0 & 0 & 0 & 0 \\
\sqrt{2} & 0 & 0 & 0 \\
0 & \sqrt{6} & 0 & 0 \\
0 & 0 & \sqrt{12} & 0 \\
0 & 0 & 0 & \sqrt{20}
\end{array}\right),
\end{aligned}
$$

which gives

$$
\begin{aligned}
\bar{\Delta}_{5 / 2} \boldsymbol{T}\left(\frac{5}{2}, \frac{3}{2}\right) \cdot \boldsymbol{\pi} \Delta= & -2 \bar{\Delta}_{5 / 2}^{+++} \Delta^{++} \pi^{+}-\frac{2}{\sqrt{5}} \bar{\Delta}_{5 / 2}^{++}\left(\sqrt{3} \Delta^{+} \pi^{+}-\sqrt{2} \Delta^{++} \pi^{0}\right) \\
& -\sqrt{\frac{2}{5}} \bar{\Delta}_{5 / 2}^{+}\left(\sqrt{3} \Delta^{0} \pi^{+}-\sqrt{6} \Delta^{+} \pi^{0}-\Delta^{++} \pi^{-}\right)-\sqrt{\frac{2}{5}} \bar{\Delta}_{5 / 2}^{0}\left(\Delta^{-} \pi^{+}-\sqrt{6} \Delta^{0} \pi^{0}-\sqrt{3} \Delta^{-} \pi^{-}\right) \\
& +\frac{2}{\sqrt{5}} \bar{\Delta}_{5 / 2}^{-}\left(\sqrt{2} \Delta^{-} \pi^{+}+\sqrt{3} \Delta^{0} \pi^{-}\right)+2 \bar{\Delta}_{5 / 2}^{-} \Delta^{-} \pi^{-}
\end{aligned}
$$

Since 35-plet is the highest multiplet among pentaquark multiplets, some resonances located in the boundary of the weight diagram have unique decay channels that do not suffer from mixing with other multiplets due to their quantum numbers. In particular, $X^{-}\left(X^{--}\right)$decays into $\bar{K}^{0} \Omega^{-}\left(K^{-} \Omega^{-}\right)$and this is the unique mode that can be searched in experiments. Similarly, the isotensor $(I=2) \Theta^{++}, \Theta^{-}$as well as $\Delta_{5 / 2}^{+++}$, $\Delta_{5 / 2}^{--}$with $I=5 / 2$ can be measured without suffering from the mixing. In addition, $\Theta_{2}$ can decay into $K \Delta$. This isospin selection rule may be useful to identify the $Y=2$ baryons. Namely, $\Theta^{+}$(and its higher spin resonances) can decay into $K N$ only, while $\Theta_{2}$ can decay into $K \Delta$ only. But $\Theta_{1}$ can decay into both $K N$ and $K \Delta$, if energetically allowed. 


\section{Interactions with pentaquark baryons}

Now we consider the interactions among pentaquarks with meson octet. These interactions can be constructed by noting that

$$
\begin{aligned}
\mathbf{8} \otimes \mathbf{8} & =\mathbf{2 7} \oplus \mathbf{1 0} \oplus \overline{\mathbf{1 0}} \oplus \mathbf{8}_{1} \oplus \mathbf{8}_{2} \oplus \mathbf{1}, \\
\mathbf{1 0} \otimes \mathbf{8} & =\mathbf{3 5} \oplus \mathbf{2 7} \oplus \mathbf{1 0} \oplus \mathbf{8} \\
\overline{\mathbf{1 0}} \otimes \mathbf{8} & =\overline{\mathbf{3 5}} \oplus \mathbf{2 7} \oplus \overline{\mathbf{1 0}} \oplus \mathbf{8} \\
\mathbf{2 7} \otimes \mathbf{8} & =\mathbf{6 4} \oplus \mathbf{3 5} \oplus \overline{\mathbf{3 5}} \oplus \mathbf{2} \mathbf{7}_{1} \oplus \mathbf{2 7} \mathbf{7}_{2} \oplus \mathbf{1 0} \oplus \overline{\mathbf{1 0}} \oplus \mathbf{8} \\
\mathbf{3 5} \otimes \mathbf{8} & =\mathbf{8 1} \oplus \mathbf{6 4} \oplus \mathbf{3 5} \mathbf{5}_{1} \oplus \mathbf{3 5}_{2} \oplus \mathbf{2 8} \oplus \mathbf{2 7} \oplus \mathbf{1 0}
\end{aligned}
$$

Thus we can see the followings. First, the pentaquark singlet can couple to pentaquark octet only. Second, the 27-27 and 35-35 interactions have two types like 8-8 interaction. Third, the interactions including 10- $\overline{\mathbf{1 0}}, \mathbf{3 5 - 8}$, and 35- $\overline{\mathbf{1 0}}$ are not allowed as they cannot form SU(3)-invariant interactions. Thus, 35-plet couplings are limited to the interactions with 27-plet and decuplet.

\section{1. $8-8$}

This interaction Lagrangian contains the well-known $f$-and $d$-type interactions as in Eq. (4.9):

$$
\mathcal{L}_{\mathbf{8 - 8}} / g_{\mathbf{8 - 8}}=(d+f) \bar{P}_{i}^{l} M_{l}^{k} P_{k}^{i}+(d-f) \bar{P}_{i}^{l} P_{l}^{k} M_{k}^{i},
$$

so that

$$
\begin{aligned}
\mathcal{L}_{\mathbf{8}-\mathbf{8}} / g_{\mathbf{8}-\mathbf{8}}== & \frac{1}{\sqrt{2}}(d+f) \bar{N}_{8} \boldsymbol{\tau} \cdot \boldsymbol{\pi} N_{8}-\frac{1}{\sqrt{6}}(d-3 f) \bar{N}_{8} N_{8} \eta-\frac{1}{\sqrt{6}}(d+3 f) \bar{N}_{8} K \Lambda_{8}^{0} \\
& +\frac{1}{\sqrt{2}}(d-f) \bar{N}_{8} \boldsymbol{\tau} \cdot \boldsymbol{\Sigma}_{8} K-i \sqrt{2} f\left(\overline{\boldsymbol{\Sigma}}_{8} \times \boldsymbol{\Sigma}_{8}\right) \cdot \boldsymbol{\pi}+\sqrt{\frac{2}{3}} d \bar{\Sigma}_{8} \Sigma_{8} \eta \\
& +\sqrt{\frac{2}{3}} d \overline{\boldsymbol{\Sigma}}_{8} \cdot \boldsymbol{\pi} \Lambda_{8}^{0}-\frac{1}{\sqrt{2}}(d+f) \bar{K}_{c} \overline{\boldsymbol{\Sigma}}_{8} \cdot \boldsymbol{\tau} \Xi_{8}+\frac{1}{\sqrt{2}}(d-f) \bar{K} \overline{\boldsymbol{\Sigma}}_{8} \cdot \boldsymbol{\tau} N_{8} \\
& +\sqrt{\frac{2}{3}} d \bar{\Lambda}_{8}^{0} \boldsymbol{\Sigma}_{8} \cdot \boldsymbol{\pi}-\sqrt{\frac{2}{3}} d \bar{\Lambda}_{8}^{0} \Lambda_{8}^{0} \eta-\frac{1}{\sqrt{6}}(d-3 f) \bar{\Lambda}_{8}^{0} \bar{K}_{c} \Xi_{8} \\
& -\frac{1}{\sqrt{6}}(d+3 f) \bar{\Lambda}_{8}^{0} \bar{K} N_{8}-\frac{1}{\sqrt{2}}(d-f) \bar{\Xi}_{8} \boldsymbol{\tau} \cdot \boldsymbol{\pi} \Xi_{8}-\frac{1}{\sqrt{6}}(d+3 f) \bar{\Xi}_{8} \Xi_{8} \eta \\
& -\frac{1}{\sqrt{2}}(d+f) \bar{\Xi}_{8} \boldsymbol{\tau} \cdot \boldsymbol{\Sigma}_{8} K_{c}-\frac{1}{\sqrt{6}}(d-3 f) \bar{\Xi}_{8} K_{c} \Lambda_{8}^{0} .
\end{aligned}
$$

Note that the values of $d$ and $f$ are different from those in $\mathcal{L}_{\mathbf{8 - 8 _ { 3 }}}$ of Eq. (4.9).

\section{2. $10-10$}

The interaction Lagrangian reads

$$
\mathcal{L}_{\mathbf{1 0 - 1 0}}=g_{\mathbf{1 0 - 1 0}} \bar{D}^{j k l} D_{m k l} M_{j}^{m}
$$

which gives

$$
\begin{aligned}
\mathcal{L}_{\mathbf{1 0 - 1 0}} / g_{\mathbf{1 0 - 1 0}}= & \sqrt{\frac{15}{2}} \bar{\Delta}_{10} \boldsymbol{T}\left(\frac{3}{2}, \frac{3}{2}\right) \cdot \boldsymbol{\pi} \Delta_{10}+\sqrt{6} \bar{\Delta}_{10} \Delta_{10} \eta-\sqrt{6} \bar{\Delta}_{10} \boldsymbol{T}\left(\frac{3}{2}, \frac{1}{2}\right) \cdot \boldsymbol{\Sigma}_{10} K \\
& -i 2 \sqrt{2}\left(\overline{\boldsymbol{\Sigma}}_{10} \times \boldsymbol{\Sigma}_{10}\right) \cdot \boldsymbol{\pi}-2 \sqrt{2} \bar{K}_{c} \overline{\boldsymbol{\Sigma}}_{10} \cdot \boldsymbol{\tau} \Xi_{10}+\sqrt{6} \bar{K} \boldsymbol{T}\left(\frac{1}{2}, \frac{3}{2}\right) \cdot \overline{\boldsymbol{\Sigma}}_{10} \Delta_{10} \\
& +\sqrt{2} \bar{\Xi}_{10} \boldsymbol{\tau} \cdot \boldsymbol{\pi} \Xi_{10}-\sqrt{6} \bar{\Xi}_{10} \Xi_{10} \eta-2 \sqrt{3} \bar{\Xi}_{10} K \Omega_{10}^{-} \\
& -2 \sqrt{2} \bar{\Xi}_{10} \boldsymbol{\tau} \cdot \boldsymbol{\Sigma}_{10} K_{c}-2 \sqrt{6} \bar{\Omega}_{10}^{-} \Omega_{10}^{-} \eta-2 \sqrt{3} \bar{\Omega}_{10}^{-} \bar{K} \Xi_{10}
\end{aligned}
$$




\section{3. $\overline{\mathbf{1 0}}-\overline{\mathbf{1 0}}$}

The interaction Lagrangian reads

$$
\mathcal{L}_{\overline{\mathbf{1 0}}-\overline{\mathbf{1 0}}}=g_{\overline{\mathbf{1 0}}-\overline{\mathbf{1 0}}} \bar{T}_{j k l} T^{m k l} M_{m}^{j}
$$

which gives

$$
\begin{aligned}
\mathcal{L}_{\overline{\mathbf{1 0}}-\overline{\mathbf{1 0}}} / g_{\overline{\mathbf{1 0}}-\overline{\mathbf{1 0}}}= & -2 \sqrt{6} \bar{\Theta} \Theta \eta-2 \sqrt{3} \bar{\Theta} \bar{K}_{c} N_{\overline{10}}-2 \sqrt{3} \bar{N}_{\overline{10}} K_{c} \Theta-\sqrt{2} \bar{N}_{\overline{10}} \boldsymbol{\tau} \cdot \boldsymbol{\pi} N_{\overline{10}} \\
& -\sqrt{6} \bar{N}_{\overline{10}} N_{\overline{10}} \eta+2 \sqrt{2} \bar{N}_{\overline{10}} \boldsymbol{\tau} \cdot \boldsymbol{\Sigma}_{\overline{10}} K+2 \sqrt{2} \bar{K} \overline{\boldsymbol{\Sigma}}_{\overline{10}} \cdot \boldsymbol{\tau} N_{\overline{10}} \\
& +i 2 \sqrt{2}\left(\overline{\boldsymbol{\Sigma}}_{\overline{10}} \times \boldsymbol{\Sigma}_{\overline{10}}\right) \cdot \boldsymbol{\pi}+\sqrt{6} \bar{K}_{c} \overline{\boldsymbol{\Sigma}}_{\overline{10}} \cdot \boldsymbol{T}\left(\frac{1}{2}, \frac{3}{2}\right) \Xi_{\overline{10}, 3 / 2} \\
& -\sqrt{6} \bar{\Xi}_{\overline{10}, 3 / 2} \boldsymbol{T}\left(\frac{3}{2}, \frac{1}{2}\right) \cdot \boldsymbol{\Sigma}_{\overline{10}} K_{c}+\sqrt{6} \bar{\Xi}_{\overline{10}, 3 / 2} \Xi_{\overline{10}, 3 / 2} \eta \\
& -\sqrt{\frac{15}{2}} \bar{\Xi}_{\overline{10}, 3 / 2} \boldsymbol{T}\left(\frac{3}{2}, \frac{3}{2}\right) \cdot \boldsymbol{\pi} \Xi_{\overline{10}, 3 / 2} .
\end{aligned}
$$

\section{4. $\quad 27-27$}

This interaction contains two types as there are two ways to fully contract the upper and lower indices. As in the case of $\mathcal{L}_{8-8}$ we define $f$ - and $d$-type interactions as

$$
\mathcal{L}_{\mathbf{2 7 - 2 7}} / g_{\mathbf{2 7 - 2 7}}=(d+f) \bar{T}_{i j}^{k l} T_{k m}^{i j} M_{l}^{m}+(d-f) \bar{T}_{i j}^{k l} T_{k l}^{i m} M_{m}^{j}
$$

Then we have

$$
\mathcal{L}_{27-27} / g_{\mathbf{2 7 - 2 7}}=\mathcal{L}_{|\Delta S|=0}^{27-27}+\mathcal{L}_{|\Delta S|=1}^{27-27}
$$

where

$$
\begin{aligned}
\mathcal{L}_{|\Delta S|=0}^{\mathbf{2 7 - 2 7}}= & -\frac{2 \sqrt{6}}{3}(d-3 f) \bar{\Theta}_{1} \Theta_{1} \eta+i 2 \sqrt{2}(d+f)\left(\overline{\boldsymbol{\Theta}}_{1} \times \boldsymbol{\Theta}_{1}\right) \cdot \boldsymbol{\pi}+\frac{\sqrt{6}}{3}(d+3 f) \bar{\Delta}_{27} \Delta_{27} \eta \\
& +\sqrt{\frac{5}{6}}(d+3 f) \bar{\Delta}_{27} \boldsymbol{T}\left(\frac{3}{2}, \frac{3}{2}\right) \cdot \boldsymbol{\pi} \Delta_{27}+\frac{8}{\sqrt{30}} d \bar{\Delta}_{27} \boldsymbol{T}\left(\frac{3}{2}, \frac{1}{2}\right) \cdot \boldsymbol{\pi} N_{27}-\frac{8}{\sqrt{30}} d \bar{N}_{27} \boldsymbol{T}\left(\frac{1}{2}, \frac{3}{2}\right) \cdot \boldsymbol{\pi} \Delta_{27} \\
& -\frac{\sqrt{6}}{15}(13 d-15 f) \bar{N}_{27} N_{27} \eta+\frac{\sqrt{2}}{15}(19 d+15 f) \bar{N}_{27} \boldsymbol{\tau} \cdot \boldsymbol{\pi} N_{27}+\frac{4 \sqrt{6}}{3} d \bar{\Sigma}_{27,2} \Sigma_{27,2} \eta \\
& +\frac{4 \sqrt{3}}{\sqrt{5}} f \bar{\Sigma}_{27,2} \boldsymbol{T}(2,2) \cdot \boldsymbol{\pi} \Sigma_{27,2}+\frac{4}{\sqrt{15}} d \bar{\Sigma}_{27,2} \boldsymbol{T}(2,1) \cdot \boldsymbol{\pi} \Sigma_{27}^{c}-\frac{4}{\sqrt{15}} d \bar{\Sigma}_{27}^{c} \boldsymbol{T}(1,2) \cdot \boldsymbol{\pi} \Sigma_{27,2} \\
& -\frac{4 \sqrt{6}}{15} d \bar{\Sigma}_{27} \Sigma_{27} \eta-i 2 \sqrt{2} f\left(\overline{\boldsymbol{\Sigma}}_{27} \times \boldsymbol{\Sigma}_{27}\right) \cdot \boldsymbol{\pi}+\frac{16 \sqrt{6}}{15} d \overline{\boldsymbol{\Sigma}}_{27} \cdot \boldsymbol{\pi} \Lambda_{27}^{0}+\frac{16 \sqrt{6}}{15} d \bar{\Lambda}_{27}^{0} \boldsymbol{\pi} \cdot \boldsymbol{\Sigma}_{27} \\
& -\frac{16 \sqrt{6}}{15} d \bar{\Lambda}_{27}^{0} \Lambda_{27}^{0} \eta+\sqrt{\frac{2}{3}}(d-3 f) \bar{\Xi}_{27,3 / 2} \Xi_{27,3 / 2} \eta-\sqrt{\frac{5}{6}}(d-3 f) \bar{\Xi}_{27,3 / 2} \boldsymbol{T}\left(\frac{3}{2}, \frac{3}{2}\right) \cdot \boldsymbol{\pi} \Xi_{27,3 / 2} \\
& +\frac{8}{\sqrt{30}} d \bar{\Xi}_{27,3 / 2} \boldsymbol{T}\left(\frac{3}{2}, \frac{1}{2}\right) \cdot \boldsymbol{\pi} \Xi_{27}-\frac{8}{\sqrt{30}} d \bar{\Xi}_{27} \boldsymbol{T}\left(\frac{1}{2}, \frac{3}{2}\right) \cdot \boldsymbol{\pi} \Xi_{27,3 / 2}-\frac{\sqrt{2}}{15}(19 d-15 f) \bar{\Xi}_{27} \boldsymbol{\tau} \cdot \boldsymbol{\pi} \Xi_{27} \\
& -\frac{\sqrt{6}}{15}(13 d+15 f) \bar{\Xi}_{27} \Xi_{27} \eta-\frac{2 \sqrt{2}}{\sqrt{3}}(d+3 f) \bar{\Omega}_{27,1} \Omega_{27,1} \eta+i 2 \sqrt{2}(d-f)\left(\overline{\boldsymbol{\Omega}}_{27,1} \times \boldsymbol{\Omega}_{27,1}\right) \cdot \boldsymbol{\pi},
\end{aligned}
$$


and

$$
\begin{aligned}
\mathcal{L}_{|\Delta S|=1}^{\mathbf{2 7 - 2 7}}= & 2(d-f) \bar{K}_{c} \overline{\boldsymbol{\Theta}}_{1} \cdot \boldsymbol{T}\left(\frac{1}{2}, \frac{3}{2}\right) \Delta_{27}-2(d-f) \bar{\Delta}_{27} \boldsymbol{T}\left(\frac{3}{2}, \frac{1}{2}\right) \cdot \boldsymbol{\Theta}_{1} K_{c}-\frac{2}{\sqrt{15}}(d+5 f) \bar{K}_{c} \overline{\boldsymbol{\Theta}}_{1} \cdot \boldsymbol{\tau} N_{27} \\
& -\frac{2}{\sqrt{15}}(d+5 f) \bar{N}_{27} \boldsymbol{\tau} \cdot \boldsymbol{\Theta}_{1} K_{c}-\sqrt{3}(d-f) \bar{\Delta}_{27} \overline{\boldsymbol{K}}_{c} \cdot \boldsymbol{I}\left(\frac{3}{2}, 2\right) \Sigma_{27,2}-\sqrt{3}(d-f) \bar{\Sigma}_{27,2} \boldsymbol{I}\left(2, \frac{3}{2}\right) \cdot \boldsymbol{K}_{c} \Delta_{27} \\
& -\frac{1}{\sqrt{5}}(3 d+5 f) \bar{\Delta}_{27} \boldsymbol{T}\left(\frac{3}{2}, \frac{1}{2}\right) \cdot \boldsymbol{\Sigma}_{27} K+\frac{1}{\sqrt{5}}(3 d+5 f) \bar{K}_{27} \cdot \boldsymbol{T}\left(\frac{1}{2}, \frac{3}{2}\right) \Delta_{27}-\frac{4}{5}(d+5 f) \bar{N}_{27} K \Lambda_{27}^{0} \\
& -\frac{4}{5}(d+5 f) \bar{\Lambda}_{27}^{0} \bar{K} N+\frac{4 \sqrt{3}}{15}(3 d-5 f) \bar{N}_{27} \boldsymbol{\tau} \cdot \boldsymbol{\Sigma}_{27} K+\frac{4 \sqrt{3}}{15}(3 d-5 f) \bar{K} \overline{\boldsymbol{\Sigma}}_{27} \cdot \boldsymbol{\tau} N_{27} \\
& -\sqrt{3}(d+f) \bar{\Sigma}_{27,2} \boldsymbol{I}\left(2, \frac{3}{2}\right) \cdot \boldsymbol{K} \Xi_{27,3 / 2}-\sqrt{3}(d+f) \bar{\Xi}_{27,3 / 2} \overline{\boldsymbol{K}} \cdot \boldsymbol{I}\left(\frac{3}{2}, 2\right) \Sigma_{27,2} \\
& +\frac{1}{\sqrt{5}}(3 d-5 f) \bar{K}_{c} \overline{\boldsymbol{\Sigma}}_{27} \cdot \boldsymbol{T}\left(\frac{1}{2}, \frac{3}{2}\right) \Xi_{27,3 / 2}-\frac{1}{\sqrt{5}}(3 d-5 f) \bar{\Xi}_{27,3 / 2} \boldsymbol{T}\left(\frac{3}{2}, \frac{1}{2}\right) \cdot \boldsymbol{\Sigma}_{27} K_{c} \\
& -\frac{4 \sqrt{3}}{15}(3 d+5 f) \bar{K}_{c} \overline{\boldsymbol{\Sigma}}_{27} \cdot \boldsymbol{\tau} \Xi_{27}-\frac{4 \sqrt{3}}{15}(3 d+5 f) \bar{\Xi}_{27} \boldsymbol{\tau} \cdot \boldsymbol{\Sigma}_{27} K_{c}-\frac{4}{5}(d-5 f) \bar{\Lambda}_{27}^{0} \bar{K}_{c} \Xi_{27} \\
& -\frac{4}{5}(d-5 f) \bar{\Xi}_{27} K_{c} \Lambda_{27}^{0}-2(d+f) \bar{\Xi}_{27,3 / 2} \boldsymbol{T}\left(\frac{3}{2}, \frac{1}{2}\right) \cdot \boldsymbol{\Omega}_{27,1} K+2(d+f) \bar{K} \overline{\boldsymbol{\Omega}}_{27,1} \cdot \boldsymbol{T}\left(\frac{1}{2}, \frac{3}{2}\right) \Xi_{27,3 / 2} \\
& +\frac{2}{\sqrt{15}}(d-5 f) \bar{\Xi}_{27} \boldsymbol{\tau} \cdot \boldsymbol{\Omega}_{27,1} K+\frac{2}{\sqrt{15}}(d-5 f) \bar{K}_{27,1} \cdot \boldsymbol{\tau} \Xi_{27} .
\end{aligned}
$$

Here,

$$
\begin{aligned}
\boldsymbol{I}\left(\frac{3}{2}, 2\right) \cdot \overline{\boldsymbol{K}}_{c} & =K^{0} I_{3 / 2,2}^{1 / 2}-K^{+} I_{3 / 2,2}^{-1 / 2}, \\
\boldsymbol{T}(2,2) \cdot \boldsymbol{\pi} & =-T_{2,2}^{(+1)} \pi^{+}+T_{2,2}^{(-1)} \pi^{-}+T_{2,2}^{(0)} \pi^{0}
\end{aligned}
$$

with

$$
I_{3 / 2,2}^{1 / 2}=\sqrt{\frac{2}{3}}\left(\begin{array}{ccccc}
2 & 0 & 0 & 0 & 0 \\
0 & \sqrt{3} & 0 & 0 & 0 \\
0 & 0 & \sqrt{2} & 0 & 0 \\
0 & 0 & 0 & 1 & 0
\end{array}\right), \quad I_{3 / 2,2}^{-1 / 2}=\sqrt{\frac{2}{3}}\left(\begin{array}{ccccc}
0 & 1 & 0 & 0 & 0 \\
0 & 0 & \sqrt{2} & 0 & 0 \\
0 & 0 & 0 & \sqrt{3} & 0 \\
0 & 0 & 0 & 0 & 2
\end{array}\right)
$$

and

$$
\begin{aligned}
& T_{2,2}^{+1}=-\sqrt{\frac{5}{6}}\left(\begin{array}{ccccc}
0 & \sqrt{2} & 0 & 0 & 0 \\
0 & 0 & \sqrt{3} & 0 & 0 \\
0 & 0 & 0 & \sqrt{3} & 0 \\
0 & 0 & 0 & 0 & \sqrt{2} \\
0 & 0 & 0 & 0 & 0
\end{array}\right), T_{2,2}^{0}=\sqrt{\frac{5}{6}}\left(\begin{array}{ccccc}
2 & 0 & 0 & 0 & 0 \\
0 & 1 & 0 & 0 & 0 \\
0 & 0 & 0 & 0 & 0 \\
0 & 0 & 0 & -1 & 0 \\
0 & 0 & 0 & 0 & -2
\end{array}\right), \\
& T_{2,2}^{-1}=\sqrt{\frac{5}{6}}\left(\begin{array}{ccccc}
0 & 0 & 0 & 0 & 0 \\
\sqrt{2} & 0 & 0 & 0 & 0 \\
0 & \sqrt{3} & 0 & 0 & 0 \\
0 & 0 & \sqrt{3} & 0 & 0 \\
0 & 0 & 0 & \sqrt{2} & 0
\end{array}\right) .
\end{aligned}
$$


Therefore, we have

$$
\begin{aligned}
\bar{\Delta}_{27} \boldsymbol{I}\left(\frac{3}{2}, 2\right) \cdot \overline{\boldsymbol{K}}_{c} \Sigma_{27,2}= & \sqrt{\frac{2}{3}} \bar{\Delta}^{++}\left(2 \Sigma_{27,2}^{++} K^{0}-\Sigma_{27,2}^{+} K^{+}\right)+\sqrt{\frac{2}{3}} \bar{\Delta}^{+}\left(\sqrt{3} \Sigma_{27,2}^{+} K^{0}-\sqrt{2} \Sigma_{27,2}^{0} K^{+}\right) \\
& +\sqrt{\frac{2}{3}} \bar{\Delta}^{0}\left(\sqrt{2} \Sigma_{27,2}^{0} K^{0}-\sqrt{3} \Sigma_{27,2}^{-} K^{+}\right)+\sqrt{\frac{2}{3}} \bar{\Delta}^{-}\left(\Sigma_{27,2}^{-} K^{0}-2 \Sigma_{27,2}^{--} K^{+}\right) \\
\bar{\Sigma}_{27,2} \boldsymbol{T}(2,2) \cdot \boldsymbol{\pi} \Sigma_{27,2}= & \sqrt{\frac{5}{6}} \bar{\Sigma}_{27,2}^{++}\left(\sqrt{2} \Sigma_{27,2}^{+} \pi^{+}+2 \Sigma_{27,2}^{++} \pi^{0}\right)+\sqrt{\frac{5}{6}} \bar{\Sigma}_{27,2}^{+}\left(\sqrt{3} \Sigma_{27,2}^{0} \pi^{+}+\Sigma_{27,2}^{+} \pi^{0}+\sqrt{2} \Sigma_{27,2}^{++} \pi^{-}\right) \\
& +\sqrt{\frac{5}{6}} \bar{\Sigma}_{27,2}^{0}\left(\sqrt{3} \Sigma_{27,2}^{-} \pi^{+}+\sqrt{3} \Sigma_{27,2}^{+} \pi^{-}\right)+\sqrt{\frac{5}{6}} \bar{\Sigma}_{27,2}^{+}\left(\sqrt{2} \Sigma_{27,2}^{--} \pi^{+}-\Sigma_{27,2}^{+} \pi^{0}+\sqrt{3} \Sigma_{27,2}^{0} \pi^{-}\right) \\
& -\sqrt{\frac{5}{6}} \bar{\Sigma}_{27,2}^{--}\left(2 \Sigma_{27,2}^{--} \pi^{0}-\sqrt{2} \Sigma_{27,2}^{-} \pi^{-}\right) .
\end{aligned}
$$

\section{5. $\quad \mathbf{3 5 - 3 5}$}

This interaction Lagrangian also contains $f$ - and $d$-type and reads

$$
\begin{aligned}
\mathcal{L}_{\mathbf{3 5 - 3 5}} / g_{\mathbf{3 5 - 3 5}} & =(d+f) \bar{T}_{a}^{i j k l} T_{i j k m}^{a} M_{l}^{m}+(d-f) \bar{T}_{a}^{i j k l} T_{i j k l}^{m} M_{m}^{a} \\
& =\mathcal{L}_{|\Delta S|=0}^{\mathbf{3 5 - 3 5}}+\mathcal{L}_{|\Delta S|=1}^{\mathbf{3 5 - 3 5}},
\end{aligned}
$$

where

$$
\begin{aligned}
\mathcal{L}_{|\Delta S|=0}^{\mathbf{3 5 - 3 5}}= & -4 \sqrt{6}(d-3 f) \bar{\Theta}_{2} \Theta_{2} \eta+\frac{6 \sqrt{12}}{\sqrt{5}}(d+f) \bar{\Theta}_{2} \boldsymbol{T}(2,2) \cdot \boldsymbol{\pi} \Theta_{2}+8 \sqrt{6} d \bar{\Delta}_{5 / 2} \Delta_{5 / 2} \eta \\
& +\frac{24 \sqrt{7}}{\sqrt{15}} f \bar{\Delta}_{5 / 2} \boldsymbol{T}\left(\frac{5}{2}, \frac{5}{2}\right) \cdot \boldsymbol{\pi} \Delta_{5 / 2}+\frac{5 \sqrt{30}}{9}(35 d+27 f) \bar{\Delta}_{35} \boldsymbol{T}\left(\frac{3}{2}, \frac{3}{2}\right) \cdot \boldsymbol{\pi} \Delta_{35} \\
& +\sqrt{\frac{6}{5}}(5 d-3 f) \bar{\Delta}_{5 / 2} \boldsymbol{T}\left(\frac{5}{2}, \frac{3}{2}\right) \cdot \boldsymbol{\pi} \Delta_{35}-\sqrt{\frac{6}{5}}(5 d-3 f) \bar{\Delta}_{35} \boldsymbol{T}\left(\frac{3}{2}, \frac{5}{2}\right) \cdot \boldsymbol{\pi} \Delta_{5 / 2} \\
& -\frac{3 \sqrt{6}}{2}(3 d-5 f) \bar{\Delta}_{35} \Delta_{35} \eta+\sqrt{6}(5 d-3 f) \bar{\Sigma}_{35,2} \Sigma_{35,2} \eta \\
& -\frac{3 \sqrt{3}}{\sqrt{5}}(d+7 f) \bar{\Sigma}_{35,2} \boldsymbol{T}(2,2) \cdot \boldsymbol{\pi} \Sigma_{35,2}+\sqrt{3}(5 d-3 f) \bar{\Sigma}_{35,2} \boldsymbol{T}(2,1) \cdot \boldsymbol{\pi} \Sigma_{35}^{c} \\
& -\sqrt{3}(5 d-3 f) \bar{\Sigma}_{35}^{c} \boldsymbol{T}(2,1) \cdot \boldsymbol{\pi} \Sigma_{35,2}-\frac{i}{\sqrt{2}}(17 d+9 f)\left(\overline{\boldsymbol{\Sigma}}_{35} \times \boldsymbol{\Sigma}_{35}\right) \cdot \boldsymbol{\pi} \\
& -\sqrt{6}(5 d-3 f) \bar{\Sigma}_{35} \Sigma_{35} \eta+2 \sqrt{6}(d-3 f) \bar{\Xi}_{35,3 / 2} \Xi_{35,3 / 2} \eta \\
& -\sqrt{30}(d-3 f) \bar{\Xi}_{35,3 / 2} \boldsymbol{T}\left(\frac{3}{2}, \frac{3}{2}\right) \cdot \boldsymbol{\pi} \Xi_{35,3 / 2}+\sqrt{3}(5 d-3 f) \bar{\Xi}_{35,3 / 2} \boldsymbol{T}\left(\frac{3}{2}, \frac{1}{2}\right) \cdot \boldsymbol{\pi} \Xi_{35} \\
& -\sqrt{3}(5 d-3 f) \bar{\Xi}_{35} \boldsymbol{T}\left(\frac{3}{2}, \frac{1}{2}\right) \cdot \boldsymbol{\pi} \Xi_{35,3 / 2}+\frac{1}{\sqrt{2}}(11 d+3 f) \bar{\Xi}_{35} \boldsymbol{\tau} \cdot \boldsymbol{\pi} \Xi_{35} \\
& -\frac{\sqrt{6}}{2}(11 d+3 f) \bar{\Xi}_{35} \Xi_{35} \eta+i 3 \sqrt{2}(3 d-5 f)\left(\overline{\boldsymbol{\Omega}}_{35,1} \times \boldsymbol{\Omega}_{35,1}\right) \cdot \boldsymbol{\pi} \\
& -\sqrt{6}(d+9 f) \Omega_{35,1} \Omega_{35,1} \eta+2 \sqrt{3}(5 d-3 f) \overline{\boldsymbol{\Omega}}_{35,1} \cdot \boldsymbol{\pi} \Omega_{35}^{-}+2 \sqrt{3}(5 d-3 f) \bar{\Omega}_{35}^{-} \boldsymbol{\pi} \cdot \boldsymbol{\Omega}_{35,1} \\
& -6 \sqrt{6}(d+f) \bar{\Omega}_{35}^{-} \Omega_{35}^{-} \eta-4 \sqrt{6}(d+3 f) \bar{X}_{X} \eta-12 \sqrt{2}(d-f) \overline{\boldsymbol{\tau}} \cdot \boldsymbol{\pi} X .
\end{aligned}
$$


and

$$
\begin{aligned}
\mathcal{L}_{|\Delta S|=1}^{\mathbf{3 5 - 3 5}=}- & \frac{24 \sqrt{3}}{\sqrt{10}}(d-f) \bar{\Theta}_{2} \boldsymbol{I}\left(2, \frac{5}{2}\right) \cdot \boldsymbol{K} \Delta_{5 / 2}+\frac{24 \sqrt{3}}{\sqrt{10}}(d-f) \bar{\Delta}_{25} \overline{\boldsymbol{K}} \cdot \boldsymbol{I}\left(\frac{5}{2}, 2\right) \Theta_{2} \\
& -\frac{3}{\sqrt{5}}(d+9 f) \bar{\Theta}_{2} \boldsymbol{I}\left(2, \frac{3}{2}\right) \cdot \boldsymbol{K} \Delta_{35}-\frac{3}{\sqrt{5}}(d+9 f) \bar{\Delta}_{35} \overline{\boldsymbol{K}} \cdot \boldsymbol{I}\left(\frac{3}{2}, 2\right) \Theta_{2} \\
& -\frac{12 \sqrt{3}}{\sqrt{10}}(d+f) \bar{\Delta}_{5 / 2} \overline{\boldsymbol{K}}_{c} \cdot \boldsymbol{I}\left(\frac{5}{2}, 2\right) \Sigma_{27,2}+\frac{12 \sqrt{3}}{\sqrt{10}}(d+f) \bar{\Sigma}_{35,2} \boldsymbol{I}\left(2, \frac{5}{2}\right) \cdot \boldsymbol{K}_{c} \Delta_{5 / 2} \\
& -\frac{3}{\sqrt{20}}(19 d-21 f) \bar{\Delta}_{35} \overline{\boldsymbol{K}}_{c} \cdot \boldsymbol{I}\left(\frac{3}{2}, 2\right) \Sigma_{35,2}-\frac{3}{\sqrt{20}}(19 d-21 f) \bar{\Sigma}_{35,2} \boldsymbol{I}\left(2, \frac{3}{2}\right) \cdot \boldsymbol{K}_{c} \Delta_{35} \\
& -\frac{\sqrt{15}}{2}(d+9 f) \bar{\Delta}_{35} \boldsymbol{T}\left(\frac{3}{2}, \frac{1}{2}\right) \cdot \boldsymbol{\Sigma} K+\frac{\sqrt{15}}{2}(d+9 f) \bar{K}_{35} \cdot \boldsymbol{T}\left(\frac{1}{2}, \frac{3}{2}\right) \Delta_{35} \\
& -9(d+f) \bar{\Sigma}_{35,2} \boldsymbol{I}\left(2, \frac{3}{2}\right) \cdot \boldsymbol{K} \Xi_{35,3 / 2}-9(d+f) \bar{\Xi}_{35,3 / 2} \overline{\boldsymbol{K}} \cdot \boldsymbol{I}\left(\frac{3}{2}, 2\right) \Sigma_{35,2} \\
& -2(d+9 f) \bar{K}_{c} \overline{\boldsymbol{\Sigma}}_{35} \cdot \boldsymbol{\tau} \Xi_{35}-2(d+9 f) \bar{\Xi}_{35} \boldsymbol{\tau} \cdot \boldsymbol{\Sigma}_{35} K_{c} \\
& +\sqrt{3}(7 d-9 f) \bar{K}_{c} \overline{\boldsymbol{\Sigma}}_{35} \cdot \boldsymbol{T}\left(\frac{1}{2}, \frac{3}{2}\right) \Xi_{35,3 / 2}-\sqrt{3}(7 d-9 f) \bar{\Xi}_{35,3 / 2} \boldsymbol{T}\left(\frac{3}{2}, \frac{1}{2}\right) \cdot \boldsymbol{\Sigma}_{35} K_{c} \\
& -6 \sqrt{3}(d+f) \bar{\Xi}_{35,3 / 2} \boldsymbol{T}\left(\frac{3}{2}, \frac{1}{2}\right) \cdot \boldsymbol{\Omega}_{35,1} K+6 \sqrt{3}(d+f) \bar{K}_{35,1} \cdot \boldsymbol{T}\left(\frac{1}{2}, \frac{3}{2}\right) \Xi_{35,3 / 2} \\
& +3(3 d+5 f) \bar{\Xi}_{35} \boldsymbol{\tau} \cdot \boldsymbol{\Omega}_{35,1}, K+3(3 d+5 f) \bar{K}_{35,1} \cdot \boldsymbol{\tau} \Xi_{35} \\
& -\sqrt{6}(d+9 f) \bar{\Xi}_{35} K \Omega_{35}^{-}-\sqrt{6}(d+9 f) \bar{\Omega}_{35}^{-} \bar{K}_{35}-6 \sqrt{2}(d+f) \bar{K}_{c} \overline{\boldsymbol{\Omega}}_{35,1} \cdot \boldsymbol{\tau} X \\
& -6 \sqrt{2}(d+f) \bar{X}_{\boldsymbol{\tau}} \cdot \boldsymbol{\Omega}_{35,1} K_{c}-4 \sqrt{3}(d-3 f) \bar{\Omega}_{35}^{-} \bar{K}_{c} X-4 \sqrt{3}(d-3 f) \bar{X} K_{c} \Omega_{35}^{-} .
\end{aligned}
$$

Here,

$$
\boldsymbol{T}\left(\frac{5}{2}, \frac{5}{2}\right) \cdot \boldsymbol{\pi}=-T_{5 / 2,5 / 2}^{(+1)} \pi^{+}+T_{5 / 2,5 / 2}^{(-1)} \pi^{-}+T_{5 / 2,5 / 2}^{(0)} \pi^{0},
$$

with

$$
\begin{aligned}
T_{5 / 2,5 / 2}^{+1}=-\frac{2 \sqrt{3}}{\sqrt{35}}\left(\begin{array}{cccccc}
0 & \sqrt{5} & 0 & 0 & 0 & 0 \\
0 & 0 & 2 \sqrt{2} & 0 & 0 & 0 \\
0 & 0 & 0 & 3 & 0 & 0 \\
0 & 0 & 0 & 0 & 2 \sqrt{2} & 0 \\
0 & 0 & 0 & 0 & 0 & \sqrt{5} \\
0 & 0 & 0 & 0 & 0 & 0
\end{array}\right), T_{5 / 2,5 / 2}^{0}=\sqrt{\frac{6}{35}}\left(\begin{array}{cccccc}
5 & 0 & 0 & 0 & 0 & 0 \\
0 & 3 & 0 & 0 & 0 & 0 \\
0 & 0 & 1 & 0 & 0 & 0 \\
0 & 0 & 0 & -1 & 0 & 0 \\
0 & 0 & 0 & 0 & -3 & 0 \\
0 & 0 & 0 & 0 & 0 & -5
\end{array}\right), \\
T_{5 / 2,5 / 2}^{-1}=\frac{2 \sqrt{3}}{\sqrt{35}}\left(\begin{array}{cccccc}
0 & 0 & 0 & 0 & 0 & 0 \\
\sqrt{5} & 0 & 0 & 0 & 0 & 0 \\
0 & 2 \sqrt{2} & 0 & 0 & 0 & 0 \\
0 & 0 & 3 & 0 & 0 & 0 \\
0 & 0 & 0 & 2 \sqrt{2} & 0 & 0 \\
0 & 0 & 0 & 0 & \sqrt{5} & 0
\end{array}\right),
\end{aligned}
$$

which leads to

$$
\begin{gathered}
\bar{\Delta}_{5 / 2} \boldsymbol{T}\left(\frac{5}{2}, \frac{5}{2}\right) \cdot \boldsymbol{\pi} \Delta_{5 / 2}=\sqrt{\frac{6}{35}}\left\{\bar{\Delta}_{5 / 2}^{+++}\left(5 \Delta_{5 / 2}^{+++} \pi^{0}+\sqrt{10} \Delta_{5 / 2}^{++} \pi^{+}+\right)+\bar{\Delta}_{5 / 2}^{++}\left(\sqrt{10} \Delta_{5 / 2}^{+++} \pi^{-}+3 \Delta_{5 / 2}^{++} \pi^{0}+4 \Delta^{+} \pi^{+}\right)\right. \\
+\bar{\Delta}_{5 / 2}^{+}\left(4 \Delta_{5 / 2}^{++} \pi^{-}+\Delta_{5 / 2}^{+} \pi^{0}+3 \sqrt{2} \Delta^{0} \pi^{+}\right)+\bar{\Delta}_{5 / 2}^{0}\left(3 \sqrt{2} \Delta_{5 / 2}^{+} \pi^{-}-\Delta_{5 / 2}^{0} \pi^{0}+4 \Delta^{-} \pi^{+}\right) \\
\left.+\bar{\Delta}_{5 / 2}^{-}\left(4 \Delta_{5 / 2}^{0} \pi^{-}-3 \Delta_{5 / 2}^{-} \pi^{0}+\sqrt{10} \Delta^{--} \pi^{+}\right)+\bar{\Delta}_{5 / 2}^{--}\left(\sqrt{10} \Delta_{5 / 2}^{-} \pi^{-}-5 \Delta_{5 / 2}^{--} \pi^{0}\right)\right\} \cdot(4.73) \\
6 . \mathbf{2 7 - \overline { \mathbf { 1 0 } }}
\end{gathered}
$$

The interaction Lagrangian of $\mathbf{2 7}$ and $\overline{\mathbf{1 0}}$ reads

$$
\mathcal{L}_{\mathbf{2 7 -} \overline{\mathbf{1 0}}} / g_{\mathbf{2 7 -} \overline{\mathbf{1 0}}}=\epsilon_{i m n} \bar{T}_{k l}^{i j} T^{m k l} M_{j}^{n}+\text { (H.c.) },
$$


which gives

$$
\begin{aligned}
\mathcal{L}_{\mathbf{2 7 -} \overline{\mathbf{1 0}}} / g_{\mathbf{2 7 - \overline { 1 0 }}}= & 2 \sqrt{6} \overline{\boldsymbol{\Theta}}_{1} \cdot \boldsymbol{\pi} \Theta-2 \bar{K}_{c} \overline{\boldsymbol{\Theta}}_{1} \cdot \boldsymbol{\tau} N_{\overline{10}}+2 \sqrt{2} \bar{\Delta}_{27} \boldsymbol{T}\left(\frac{3}{2}, \frac{1}{2}\right) \cdot \boldsymbol{\pi} N_{\overline{10}} \\
& -2 \sqrt{2} \bar{\Delta}_{27} \boldsymbol{T}\left(\frac{3}{2}, \frac{1}{2}\right) \cdot \boldsymbol{\Sigma}_{\overline{10}} K-\frac{7 \sqrt{2}}{\sqrt{15}} \bar{N}_{27} \boldsymbol{\tau} \cdot \boldsymbol{\pi} N_{\overline{10}}-\frac{3 \sqrt{10}}{5} \bar{N}_{27} N_{\overline{10}} \eta \\
& +\frac{6 \sqrt{5}}{5} \bar{N}_{27} K_{c} \Theta-\frac{2 \sqrt{2}}{\sqrt{15}} \bar{N}_{27} \boldsymbol{\tau} \cdot \boldsymbol{\Sigma}_{\overline{10}} K+\frac{2 \sqrt{2}}{\sqrt{3}} \bar{\Sigma}_{27,2} \boldsymbol{T}(2,1) \cdot \boldsymbol{\pi} \Sigma_{\overline{10}}^{c} \\
& -3 \bar{\Sigma}_{27,2} \boldsymbol{I}\left(2, \frac{3}{2}\right) \cdot \boldsymbol{K} \Xi_{\overline{10}, 3 / 2}+i \frac{6}{\sqrt{5}}\left(\overline{\boldsymbol{\Sigma}}_{27} \times \boldsymbol{\Sigma}_{\overline{10}}\right) \cdot \boldsymbol{\pi}-\frac{4 \sqrt{3}}{\sqrt{5}} \bar{\Sigma}_{27} \Sigma_{\overline{10}} \eta \\
& -\frac{4}{\sqrt{5}} \bar{K} \overline{\boldsymbol{\Sigma}}_{27} \cdot \boldsymbol{\tau} N_{\overline{10}}-\sqrt{\frac{3}{5}} \bar{K}_{c} \overline{\boldsymbol{\Sigma}}_{27} \cdot \boldsymbol{T}\left(\frac{1}{2}, \frac{3}{2}\right) \Xi_{\overline{10}, 3 / 2}-\frac{8}{\sqrt{15}} \bar{\Lambda}_{27}^{0} \boldsymbol{\pi} \cdot \boldsymbol{\Sigma}_{\overline{10}} \\
& +\frac{4 \sqrt{3}}{\sqrt{5}} \bar{\Lambda}_{27}^{0} \bar{K} N_{\overline{10}}-\sqrt{\frac{5}{2}} \bar{\Xi}_{27,3 / 2} \boldsymbol{T}\left(\frac{3}{2}, \frac{3}{2}\right) \cdot \boldsymbol{\pi} \Xi_{\overline{10}, 3 / 2}-3 \sqrt{2} \bar{\Xi}_{27,3 / 2} \Xi_{\overline{10}, 3 / 2} \eta \\
& +\sqrt{2} \bar{\Xi}_{27,3 / 2} \boldsymbol{T}\left(\frac{3}{2}, \frac{1}{2}\right) \cdot \boldsymbol{\Sigma}_{\overline{10}} K_{c}+\frac{2 \sqrt{6}}{\sqrt{15}} \bar{\Xi}_{27} \boldsymbol{T}\left(\frac{1}{2}, \frac{3}{2}\right) \cdot \boldsymbol{\pi} \Xi_{\overline{10}, 3 / 2} \\
& +\frac{8 \sqrt{2}}{\sqrt{15}} \bar{\Xi}_{27} \boldsymbol{\tau} \cdot \boldsymbol{\Sigma}_{\overline{10}} K_{c}-2 \sqrt{3} \bar{K} \overline{\boldsymbol{\Omega}}_{27,1} \cdot \boldsymbol{T}\left(\frac{1}{2}, \frac{3}{2}\right) \Xi_{\overline{10}, 3 / 2} \\
& +(\text { H.c. }) .
\end{aligned}
$$

\section{7. $\quad 35-27$}

The interaction Lagrangian in this case is obtained as

$$
\mathcal{L}_{\mathbf{3 5 - 2 7}} / g_{\mathbf{3 5 - 2 7}}=\epsilon_{i m n} \bar{T}_{a}^{i j k l} T_{j k}^{m a} M_{l}^{n}+(\text { H.c. }),
$$


which gives

$$
\begin{aligned}
& \mathcal{L}_{\mathbf{3 5 - 2 7}} / g_{\mathbf{3 5 - 2 7}}=4 \sqrt{2} \bar{\Theta}_{2} \boldsymbol{T}(2,1) \cdot \boldsymbol{\pi} \Theta_{1}^{c}-3 \sqrt{2} \bar{\Theta}_{2} \boldsymbol{I}\left(2, \frac{3}{2}\right) \cdot \boldsymbol{K} \Delta_{27} \\
& -\frac{12}{\sqrt{5}} \bar{\Delta}_{5 / 2} \overline{\boldsymbol{K}}_{c} \cdot \boldsymbol{I}\left(\frac{5}{2}, 2\right) \Sigma_{27,2}+2 \sqrt{3} \bar{\Delta}_{5 / 2} \boldsymbol{T}\left(\frac{5}{2}, \frac{3}{2}\right) \cdot \boldsymbol{\pi} \Delta_{27} \\
& +\sqrt{10} \bar{\Delta}_{35} \boldsymbol{T}\left(\frac{3}{2}, \frac{1}{2}\right) \cdot \Theta_{1} K_{c}+\sqrt{\frac{3}{10}} \bar{\Delta}_{35} \overline{\boldsymbol{K}}_{c} \cdot \boldsymbol{I}\left(\frac{3}{2}, 2\right) \Sigma_{27,2} \\
& -\frac{5}{\sqrt{2}} \bar{\Delta}_{35} \boldsymbol{T}\left(\frac{3}{2}, \frac{1}{2}\right) \cdot \boldsymbol{\Sigma}_{27} K-\frac{7}{\sqrt{12}} \bar{\Delta}_{35} \boldsymbol{T}\left(\frac{3}{2}, \frac{3}{2}\right) \cdot \boldsymbol{\pi} \Delta_{27} \\
& +\frac{10}{\sqrt{3}} \bar{\Delta}_{35} \boldsymbol{T}\left(\frac{3}{2}, \frac{1}{2}\right) \cdot \boldsymbol{\pi} N_{27}-6 \bar{\Sigma}_{35,2} \Sigma_{27,2} \eta-\frac{3 \sqrt{2}}{\sqrt{5}} \bar{\Sigma}_{35,2} \boldsymbol{T}(2,2) \cdot \boldsymbol{\pi} \Sigma_{27,2} \\
& +\sqrt{10} \bar{\Sigma}_{35,2} \boldsymbol{T}(2,1) \cdot \boldsymbol{\pi} \Sigma_{27}^{c}-3 \sqrt{2} \bar{\Sigma}_{35,2} \boldsymbol{I}\left(2, \frac{3}{2}\right) \cdot \boldsymbol{K} \Xi_{27,3 / 2} \\
& +\frac{3}{\sqrt{2}} \bar{\Sigma}_{35,2} \boldsymbol{I}\left(2, \frac{3}{2}\right) \cdot \boldsymbol{K}_{c} \Delta_{27}-\frac{2 \sqrt{10}}{3} \bar{K}_{c} \overline{\boldsymbol{\Sigma}}_{35} \cdot \boldsymbol{\tau} \Xi_{27} \\
& -2 \sqrt{5} \bar{\Sigma}_{35} \Sigma_{27} \eta-\frac{5}{\sqrt{6}} \bar{K} \overline{\boldsymbol{\Sigma}}_{35} \cdot \boldsymbol{T}\left(\frac{1}{2}, \frac{3}{2}\right) \Delta_{27}-\sqrt{\frac{2}{3}} \bar{K}_{c} \overline{\boldsymbol{\Sigma}}_{35} \cdot \boldsymbol{T}\left(\frac{1}{2}, \frac{3}{2}\right) \Xi_{27,3 / 2} \\
& +i \sqrt{15}\left(\bar{\Sigma}_{35} \times \Sigma_{27}\right) \cdot \boldsymbol{\pi}+\frac{8 \sqrt{5}}{3} \bar{\Sigma}_{35} \cdot \boldsymbol{\pi} \Lambda_{27}^{0}-\frac{4 \sqrt{10}}{3} \bar{K} \bar{\Sigma}_{35} \cdot \boldsymbol{\tau} N_{27} \\
& +\frac{\sqrt{2}}{3} \bar{\Sigma}_{35}^{c} \boldsymbol{T}(1,2) \cdot \boldsymbol{\pi} \Sigma_{27,2}-4 \sqrt{3} \bar{\Xi}_{35,3 / 2} \Xi_{27,3 / 2} \eta \\
& -\frac{2 \sqrt{5}}{\sqrt{3}} \bar{\Xi}_{35,3 / 2} \boldsymbol{T}\left(\frac{3}{2}, \frac{3}{2}\right) \cdot \boldsymbol{\pi} \Xi_{27,3 / 2}+\sqrt{6} \bar{\Xi}_{35,3 / 2} \overline{\boldsymbol{K}} \cdot \boldsymbol{I}\left(\frac{3}{2}, 2\right) \Sigma_{27,2} \\
& +\sqrt{10} \bar{\Xi}_{35,3 / 2} \boldsymbol{T}\left(\frac{3}{2}, \frac{1}{2}\right) \cdot \boldsymbol{\Sigma}_{27} K_{c}+\frac{2 \sqrt{15}}{3} \Xi_{35,3 / 2} \boldsymbol{T}\left(\frac{3}{2}, \frac{1}{2}\right) \cdot \boldsymbol{\pi} \Xi_{27} \\
& -2 \sqrt{2} \bar{\Xi}_{35,3 / 2} \boldsymbol{T}\left(\frac{3}{2}, \frac{1}{2}\right) \cdot \boldsymbol{\Omega}_{27,1} K-\frac{5 \sqrt{5}}{3} \bar{\Xi}_{35} \boldsymbol{\tau} \cdot \boldsymbol{\pi} \Xi_{27} \\
& +\frac{2}{\sqrt{3}} \bar{\Xi}_{35} \boldsymbol{T}\left(\frac{1}{2}, \frac{3}{2}\right) \cdot \boldsymbol{\pi} \Xi_{27,3 / 2}+2 \sqrt{10} \bar{\Xi}_{35} K_{c} \Lambda_{27}^{0}+\frac{2 \sqrt{10}}{\sqrt{3}} \bar{\Xi}_{35} \boldsymbol{\tau} \cdot \boldsymbol{\Sigma}_{27} K_{c} \\
& -\sqrt{\frac{2}{3}} \bar{\Xi}_{35} \boldsymbol{\tau} \cdot \boldsymbol{\Omega}_{27,1} K-6 \bar{\Omega}_{35,1} \Omega_{27,1} \eta+i 2 \sqrt{3}\left(\overline{\boldsymbol{\Omega}}_{35,1} \times \boldsymbol{\Omega}_{27,1}\right) \cdot \boldsymbol{\pi} \\
& -2 \sqrt{6} \bar{K} \overline{\boldsymbol{\Omega}}_{35,1} \cdot \boldsymbol{T}\left(\frac{1}{2}, \frac{3}{2}\right) \Xi_{27,3 / 2}-\sqrt{10} \bar{K} \overline{\boldsymbol{\Omega}}_{35,1} \cdot \boldsymbol{\tau} \Xi_{27}-2 \sqrt{2} \bar{\Omega}_{35}^{-} \boldsymbol{\pi} \cdot \boldsymbol{\Omega}_{27,1} \\
& +2 \sqrt{15} \bar{\Omega}_{35}^{-} \bar{K} \Xi_{27}+4 \sqrt{3} \bar{X} \boldsymbol{\tau} \cdot \boldsymbol{\Omega}_{27,1} K_{c} \\
& + \text { (H.c.). }
\end{aligned}
$$

\section{8. $\quad$ Other interactions}

Other interactions of pentaquarks can be obtained directly from the results in the previous subsections. Namely, the interactions for $\mathbf{1 - 8}, \mathbf{1 0}-\mathbf{8}, \overline{\mathbf{1 0}} \mathbf{- 8}$, and $\mathbf{2 7 - 8}$ can be obtained from Eqs. (4.6), (4.12), (4.19), and (4.24) by replacing the three-quark octet by pentaquark octet. Also the interactions for 27-10 and 35-10 can be read from Eqs. (4.44) and (4.49), respectively, by replacing the three-quark decuplet by pentaquark decuplet. This completes the interactions of pentaquark baryons with pentaquark baryons and meson octet.

\section{MASS RELATIONS}

In this Section, we derive several relations for the mass differences among pentaquark baryons. This can be obtained by using the Gell-Mann-Okubo mass formula,

$$
M=M_{0}+\alpha Y+\beta D_{3}^{3},
$$


where $M_{0}$ is a common mass of a given multiplet and $D_{3}^{3}=I(I+1)-Y^{2} / 4-C / 6$ with $C=2(p+q)+\frac{2}{3}\left(p^{2}+p q+q^{2}\right)$ for the $(p, q)$ representation. In the tensor representation, one can obtain the same results as follows. Since all the particles belonging to an irreducible representation of $\mathrm{SU}(3)$ are degenerate in the $\mathrm{SU}(3)$ symmetry limit, it is required to include $\mathrm{SU}(3)$ symmetry breaking to obtain the mass splittings. It is well-known that the Hamiltonian which breaks $\mathrm{SU}(3)$ symmetry but still preserves the isospin symmetry and hypercharge is proportional to the Gell-Mann matrix $\lambda_{8}$. Thus we introduce the hypercharge tensor as

$$
\mathcal{Y}=\left(\begin{array}{ccc}
1 & 0 & 0 \\
0 & 1 & 0 \\
0 & 0 & -2
\end{array}\right)
$$

Then the baryon masses can be obtained by constructing all possible contractions among irreducible tensors and the hypercharge tensor. As the mass formulas contain several parameters which take different values depending on the multiplet, we can obtain only the relations between the masses. Also note that we do not consider mixing among baryon multiplets in this work.

\section{A. Octet}

For the pentaquark octet, the mass term is given by

$$
H_{\mathbf{8}}=a \bar{P}_{j}^{i} P_{i}^{j}+b \bar{P}_{j}^{i} \mathcal{Y}_{i}^{l} P_{l}^{j}+c \bar{P}_{j}^{i} \mathcal{Y}_{l}^{j} P_{i}^{l} .
$$

Using the particle identifications for the octet tensor, we obtain the octet mass as

$$
\begin{aligned}
& N_{8}=a+b-2 c, \quad \Lambda_{8}=a-b-c, \\
& \Sigma_{8}=a+b+c, \quad \Xi_{8}=a-2 b+c .
\end{aligned}
$$

The particle name in each equation indicates its mass in this Section. One can then easily find that Eq. (5.3) leads to the Gell-Mann-Okubo mass relation for the pentaquark octet,

$$
2\left(N_{8}+\Xi_{8}\right)=3 \Lambda_{8}+\Sigma_{8}
$$

\section{B. Decuplet}

In this case, the fully contracted Hamiltonian reads

$$
H_{10}=a \bar{D}^{i j k} D_{i j k}+b \bar{D}^{i j k} \mathcal{Y}_{k}^{l} D_{i j l} .
$$

Working out the contraction, we obtain

$$
\begin{array}{ll}
\Delta_{10}=6(a+b), & \Sigma_{10}=6 a, \\
\Xi_{10}=6(a-b), & \Omega_{10}=6(a-2 b) .
\end{array}
$$

Note that we have the well-known equal spacing for the decuplet spectrum,

$$
\Omega_{10}-\Xi_{10}=\Xi_{10}-\Sigma_{10}=\Sigma_{10}-\Delta_{10} .
$$

\section{Antidecuplet}

The mass spectrum of antidecuplet is very similar to that of decuplet, namely,

$$
H_{\overline{\mathbf{1 0}}}=a \bar{T}_{i j k} T^{i j k}+b \bar{T}_{i j k} \mathcal{Y}_{l}^{k} T^{i j l},
$$

which gives

$$
\begin{aligned}
& \Theta=6(a-2 b), \quad N_{\overline{10}}=6(a-b), \\
& \Sigma_{\overline{10}}=6 a, \quad \Xi_{\overline{10}, 3 / 2}=6(a+b) .
\end{aligned}
$$

Again, we have equal spacing rule in the spectrum [46],

$$
\Xi_{\overline{10}, 3 / 2}-\Sigma_{\overline{10}}=\Sigma_{\overline{10}}-N_{\overline{10}}=N_{\overline{10}}-\Theta .
$$




\section{27-plet}

There are three possible ways to contract upper and lower indices as in the case of octet. We have

$$
H_{\mathbf{2 7}}=a \bar{T}_{k l}^{i j} T_{i j}^{k l}+b \bar{T}_{k l}^{i j} \mathcal{Y}_{m}^{l} T_{i j}^{k m}+c \bar{T}_{k l}^{i j} \mathcal{Y}_{j}^{m} T_{i m}^{k l}
$$

The masses are then given by

$$
\begin{aligned}
& \Theta_{1}=4 a-8 b+4 c, \\
& \Delta_{27}=4 a-2 b+4 c, \quad N_{27}=4 a-\frac{28}{5} b+\frac{2}{5} c, \\
& \Sigma_{27,2}=4 a+4 b+4 c, \quad \Sigma_{27}=4 a-\frac{4}{5} b-\frac{4}{5} c, \quad \Lambda_{27}=4 a-\frac{16}{5} b-\frac{16}{5} c, \\
& \Xi_{27,3 / 2}=4 a+4 b-2 c, \quad \Xi_{27}=4 a+\frac{2}{5} b-\frac{28}{5} c, \\
& \Omega_{27,1}=4 a+4 b-8 c .
\end{aligned}
$$

From the above results, we can obtain several mass relations,

$$
\begin{aligned}
& 3\left(\Sigma_{27}+\Theta_{1}\right)=2\left(\Delta_{27}+2 N_{27}\right), \\
& 3\left(\Xi_{27,3 / 2}+2 \Theta_{1}\right)=4 \Delta_{27}+5 N_{27}, \quad 3\left(\Xi_{27}+2 \Theta_{1}\right)=\Delta_{27}+8 N_{27}, \\
& 3\left(\Omega_{27,1}+3 \Theta_{1}\right)=2\left(\Delta_{27}+5 N_{27}\right) .
\end{aligned}
$$

Some interesting relations can be found. First, one can find the analog of Gell-Mann-Okubo relation,

$$
2\left(N_{27}+\Xi_{27}\right)=3 \Lambda_{27}+\Sigma_{27}
$$

Second, we note that some of the 27-plet members, i.e., $\Theta_{1}, \Delta_{27}, \Sigma_{27,2}, \Xi_{27,3 / 2}$, and $\Omega_{27,1}$, satisfy two independent equal-spacing rules,

$$
\begin{aligned}
\Omega_{27,1}-\Xi_{27,3 / 2} & =\Xi_{27,3 / 2}-\Sigma_{27,2}, \\
\Sigma_{27,2}-\Delta_{27} & =\Delta_{27}-\Theta_{1} .
\end{aligned}
$$

Note that they are the states with maximum isospin for a given hypercharge and the equal-spacing rule holds independently for the upper half of the 27-plet weight diagram and for the lower half of the weight diagram (Fig. 7).

\section{E. 35-plet}

In this case, we have

$$
H_{\mathbf{3 5}}=a \bar{T}_{i}^{j k l m} T_{j k l m}^{i}+b \bar{T}_{i}^{j k l m} \mathcal{Y}_{j}^{n} T_{n k l m}^{i}+c \bar{T}_{i}^{j k l m} \mathcal{Y}_{n}^{i} T_{j k l m}^{n}
$$

The obtained masses are

$$
\begin{aligned}
& \Theta_{2}=24 a+24 b-48 c, \\
& \Delta_{5 / 2}=24 a+24 b+24 c, \quad \Delta_{35}=24 a+9 b-36 c, \\
& \Sigma_{35,2}=24 a+6 b+24 c, \quad \Sigma_{35}=24 a-6 b-24 c, \\
& \Xi_{35,3 / 2}=24 a-12 b+24 c, \quad \Xi_{35}=24 a-21 b-12 c, \\
& \Omega_{35,1}=24 a-30 b+24 c, \quad \Omega_{35}=24 a-36 b, \\
& X=24 a-48 b+24 c .
\end{aligned}
$$

Here we observe that there are two sets of baryons which satisfy the equal-spacing rule separately. Namely, $\left(\Theta_{2}, \Delta_{35}\right.$, $\left.\Sigma_{35}, \Xi_{35}, \Omega_{35}\right)$ are equally spaced and another equal spacing rule holds for $\left(\Delta_{5 / 2}, \Sigma_{35,2}, \Xi_{35,3 / 2}, \Omega_{35,1}, X\right)$,

$$
\begin{aligned}
& \Omega_{35}-\Xi_{35}=\Xi_{35}-\Sigma_{35}=\Sigma_{35}-\Delta_{35}=\Delta_{35}-\Theta_{2}, \\
& X-\Omega_{35,1}=\Omega_{35,1}-\Xi_{35,3 / 2}=\Xi_{35,3 / 2}-\Sigma_{35,2}=\Sigma_{35,2}-\Delta_{5 / 2} .
\end{aligned}
$$

One can derive other mass relations such as

$$
\begin{aligned}
& 5\left(\Theta_{2}+\Sigma_{35,2}\right)=2\left(2 \Delta_{5 / 2}+3 \Delta_{35}\right), \quad 5\left(\Sigma_{35}-\Sigma_{35,2}\right)=-4\left(\Delta_{5 / 2}-\Delta_{35}\right), \\
& 5\left(\Xi_{35}-2 \Sigma_{35,2}\right)=-8 \Delta_{5 / 2}+3 \Delta_{35}, \quad 5\left(\Omega_{35}-3 \Sigma_{35,2}\right)=-2\left(6 \Delta_{5 / 2}-\Delta_{35}\right) \text {. }
\end{aligned}
$$




\section{SUMMARY}

In summary, we have obtained the flavor wave functions of all pentaquark baryons in SU(3) quark model including $\mathbf{1}, \mathbf{8}, \mathbf{1 0}, \overline{\mathbf{1 0}}, \mathbf{2 7}$, and 35. Then the $\mathrm{SU}(3)$ symmetric Lagrangian for the interactions involving pentaquark baryons is constructed and the mass relations among the pentaquark baryons are derived. Together with the mass sum rules, the obtained SU(3) selection rules and SU(3) symmetry relations would be useful in searching for the pentaquark baryons in future experiments and studying their production processes as well as developing more sophisticated models for pentaquark structure. The flavor-spin wave function of pentaquark baryons can be constructed by extending our results to $\mathrm{SU}(6)$ [65] and the symmetry breaking effects can be introduced to our SU(3) symmetric Lagrangian in a standard way.

\section{Acknowledgments}

We are grateful to Su Houng Lee and K. Nakayama for fruitful discussions. This work was supported by Forschungszentrum-Jülich, contract No. 41445282 (COSY-058) and the Brain Korea 21 project of Korean Ministry of Education.

[1] LEPS Collaboration, T. Nakano et al., Phys. Rev. Lett. 91, 012002 (2003).

[2] DIANA Collaboration, V. V. Barmin, et al., Yad. Fiz. 66, 1763 (2003), [Phys. At. Nucl. 66, 1715 (2003)].

[3] CLAS Collaboration, S. Stepanyan et al., Phys. Rev. Lett. 91, 252001 (2003).

[4] SAPHIR Collaboration, J. Barth et al., Phys. Lett. B 572, 127 (2003).

[5] CLAS Collaboration, V. Kubarovsky et al., Talk at CIPANP 2003, New York, 2003, hep-ex/0307088.

[6] A. E. Asratyan, A. G. Dolgolenko, and M. A. Kubantsev, hep-ex/0309042.

[7] CLAS Collaboration, V. Kubarovsky et al., Phys. Rev. Lett. 92, 032001 (2004).

[8] S. Armstrong, B. Mellado, and S. L. Wu, hep-ph/0312344.

[9] HERMES Collaboration, A. Airapetian et al., Phys. Lett. B 585, 213 (2004).

[10] SVD Collaboration, A. Aleev et al., hep-ex/0401024.

[11] P. Zh. Aslanyan, V. N. Emelyanenko, and G. G. Rikhkvitzkaya, hep-ex/0403044.

[12] S. Salur, nucl-ex/0403009.

[13] C. Pinkenburg, nucl-ex/0404001.

[14] NA49 Collaboration, C. Alt et al., Phys. Rev. Lett. 92, 042003 (2004).

[15] H. G. Fischer and S. Wenig, hep-ex/0401014.

[16] WA89 Collaboration, M. I. Adamovich et al., hep-ex/0405042.

[17] J. Pochodzalla, hep-ex/0406077.

[18] H1 Collaboration, A. Aktas et al., hep-ex/0403017.

[19] A. R. Dzierba, D. Krop, M. Swat, S. Teige, and A. P. Szczepaniak, Phys. Rev. D 69, 051901 (2004).

[20] E. Golowich, Phys. Rev. D 4, 262 (1971).

[21] Particle Data Group, M. Aguilar-Benitez et al., Phys. Lett. B 170, 1 (1986).

[22] H. Gao and B.-Q. Ma, Mod. Phys. Lett. A 14, 2313 (1999).

[23] H. J. Lipkin, Phys. Lett. B 195, 484 (1987).

[24] C. Gignoux, B. Silvestre-Brac, and J. M. Richard, Phys. Lett. B 193, 323 (1987).

[25] S. Fleck, C. Gignoux, J. M. Richard, and B. Silvestre-Brac, Phys. Lett. B 220, 616 (1989); S. Zouzou and J.-M. Richard, Few-Body Syst. 16, 1 (1994).

[26] M. Genovese, J.-M. Richard, Fl. Stancu, and S. Pepin, Phys. Lett. B 425, 171 (1998); Fl. Stancu, Phys. Rev. D 58, 111501 (1998).

[27] D. O. Riska and N. N. Scoccola, Phys. Lett. B 299, 338 (1993).

[28] Y. Oh, B.-Y. Park, and D.-P. Min, Phys. Lett. B 331, 362 (1994); Phys. Rev. D 50, 3350 (1994); Y. Oh and B.-Y. Park, Phys. Rev. D 51, 5016 (1995).

[29] E791 Collaboration, E. M. Aitala, et al., Phys. Rev. Lett. 81, 44 (1998); Phys. Lett. B 448, 303 (1999).

[30] K. Cheung, hep-ph/0308176.

[31] H.-Y. Cheng, C.-K. Chua, and C.-W. Hwang, hep-ph/0403232; X.-G. He and X.-Q. Li, hep-ph/0403191.

[32] T. E. Browder, I. R. Klebanov, and D. R. Marlow, Phys. Lett. B 587, 62 (2004).

[33] I. W. Stewart, M. E. Wessling, and M. B. Wise, hep-ph/0402076.

[34] B. Wu and B.-Q. Ma, hep-ph/0402244.

[35] H. Kim, S. H. Lee, and Y. Oh, hep-ph/0404170.

[36] T. Hirose, K. Kanai, S. Kitamura, and T. Kobayashi, Nuovo Cim. 50A, 120 (1979); C. Fukunaga, R. Hamatsu, T. Hirose, W. Kitamura, and T. Yamagata, Nuovo Cim. 58A, 199 (1980). 
[37] V. M. Karnaukhov, V. I. Moroz, C. Coca, and A. Mihul, Phys. Lett. B 281, 148 (1992).

[38] A. V. Aref'ev, et al., Yad. Fiz. 51, 414 (1990) [Sov. J. Nucl. Phys. 51, 264 (1990)].

[39] J. Amirzadeh, et al., Phys. Lett. 89B, 125 (1979).

[40] B. M. Abramov, et al., Yad. Fiz. 53, 179 (1991) [Sov. J. Nucl. Phys. 53, 114 (1991)].

[41] D. Aston, et al., Phys. Rev. D 32, 2270 (1985).

[42] L. G. Landsberg, Phys. Rep. 320, 223 (1999); SPHINX Collaboration, Yu. M. Antipov, et al., Yad. Fiz. 65, 2131 (2002) [Phys. Atom. Nucl. 65, 2070 (2002)].

[43] R. L. Jaffe, Phys. Rev. D 15, 267 (1977); D. Strottman, ibid. 20, 748 (1979); H. Hogaasen and P. Sorba, Nucl. Phys. B145, 119 (1978); M. De Crombrugghe, H. Hogaasen, and P. Sorba, ibid. B156, 347 (1979); K. Maltman and S. Godfrey, ibid. A452, 669 (1986); A. Ferrer, V. F. Perepelitsa, and A. A. Grigoryan, Z. Phys. C 56, 215 (1992).

[44] M. Chemtob, Nucl. Phys. B256, 600 (1985); A. V. Manohar, ibid. B248, 19 (1984); M. Praszałowicz, in Skyrmions and Anomalies, edited by M. Jezabeck and M. Praszałowicz, (World Scientific, Singapore, 1987), p. 112.

[45] H. Weigel, Eur. Phys. J. A 2, 391 (1998).

[46] D. Diakonov, V. Petrov, and M. Polyakov, Z. Phys. A 359, 305 (1997).

[47] M. V. Polyakov, A. Sibirtsev, K. Tsushima, W. Cassing, and K. Goeke, Eur. Phys. J. A 9, 115 (2000).

[48] H. Walliser and V. B. Kopeliovich, Zh. Eksp. Teor. Fiz. 124, 483 (2003), [J. Exp. Theor. Phys. 97, 433 (2003)].

[49] M. Praszałowicz, Phys. Lett. B 575, 234 (2003).

[50] N. Itzhaki, I. R. Klebanov, P. Ouyang, and L. Rastelli, Nucl. Phys. B684, 264 (2004).

[51] B. K. Jennings and K. Maltman, hep-ph/0308286.

[52] D. Borisyuk, M. Faber, and A. Kobushkin, hep-ph/0307370.

[53] S.-L. Zhu, Phys. Rev. Lett. 91, 232002 (2003); R. D. Matheus, F. S. Navarra, M. Nielsen, R. da Silva, and S. H. Lee, Phys. Lett. B 578, 323 (2003); J. Sugiyama, T. Doi, and M. Oka, Phys. Lett. B 581, 167 (2004); M. Eidemüller, hep-ph/0404126.

[54] T. D. Cohen, Phys. Lett. B 581, 175 (2004); T. D. Cohen and R. F. Lebed, Phys. Lett. B 578, 150 (2004).

[55] E. Jenkins and A. V. Manohar, hep-ph/0401190; hep-ph/0402024; A. V. Manohar, hep-ph/0404122.

[56] F. Csikor, Z. Fodor, S. D. Katz, and T. G. Kovács, JHEP 0311, 070 (2003).

[57] S. Sasaki, hep-lat/0310014.

[58] T.-W. Chiu and T.-H. Hsieh, hep-ph/0403020.

[59] M. Karliner and H. J. Lipkin, hep-ph/0307243.

[60] R. Jaffe and F. Wilczek, Phys. Rev. Lett. 91, 232003 (2003).

[61] Y. Oh, H. Kim, and S. H. Lee, Phys. Rev. D 69094009.

[62] F. E. Close and J. J. Dudek, Phys. Lett. B 586, 75 (2004).

[63] S. H. Lee, H. Kim, and Y. Oh, hep-ph/0402135.

[64] D. Diakonov and V. Petrov, hep-ph/0310212.

[65] R. Bijker, M. M. Giannini, and E. Santopinto, hep-ph/0310281.

[66] Fl. Stancu and D. O. Riska, Phys. Lett. B 575, 242 (2003).

[67] C. E. Carlson, C. D. Carone, H. J. Kwee, and V. Nazaryan, Phys. Lett. B 573, 101 (2003).

[68] C. E. Carlson, C. D. Carone, H. J. Kwee, and V. Nazaryan, Phys. Lett. B 579, 52 (2004).

[69] S. M. Gerasyuta and V. I. Kochkin, hep-ph/0310227.

[70] N. I. Kochelev, H. J. Lee, and V. Vento, hep-ph/0404065.

[71] M. Praszałowicz, Acta Phys. Pol. B35, 1625 (2004).

[72] B. Wu and B.-Q. Ma, Phys. Rev. D 69, 077501 (2004); Phys. Lett. B 586, 62 (2004).

[73] J. Ellis, M. Karliner, and M. Praszałowicz, hep-ph/0401127.

[74] V. Dmitrašinović and Fl. Stancu, hep-ph/0402190.

[75] W. Liu and C. M. Ko, Phys. Rev. C 68, 045203 (2003); nucl-th/0309023.

[76] S. I. Nam, A. Hosaka, and H.-C. Kim, Phys. Lett. B 579, 43 (2003).

[77] Y. Oh, H. Kim, and S. H. Lee, Phys. Rev. D 69, 014009 (2004).

[78] Q. Zhao and J. S. Al-Khalili, Phys. Lett. B 585, 91 (2004).

[79] K. Nakayama and W. G. Love, hep-ph/0404011.

[80] B.-G. Yu, T.-K. Choi, and C.-R. Ji, nucl-th/0312075.

[81] Y. Oh, H. Kim, and S. H. Lee, hep-ph/0312229.

[82] W. Liu, C. M. Ko, and V. Kubarovsky, Phys. Rev. C 69, 025202 (2004).

[83] K. Nakayama and K. Tsushima, Phys. Lett. B 583, 269 (2004).

[84] Y. Oh, H. Kim, and S. H. Lee, Phys. Rev. D 69, 074016 (2004).

[85] A. W. Thomas, K. Hicks, and A. Hosaka, Prog. Theor. Phys. 111, 291 (2004).

[86] C. Hanhart et al., hep-ph/0312236.

[87] T. Hyodo, A. Hosaka, and E. Oset, Phys. Lett. B 579, 290 (2004).

[88] C. E. Carlson, C. D. Carone, H. J. Kwee, and V. Nazaryan, hep-ph/0312325.

[89] P. Ko, J. Lee, T. Lee, and J.-H. Park, hep-ph/0312147.

[90] Y.-R. Liu, A. Zhang, P.-Z. Huang, W.-Z. Deng, X.-L. Chen, and S.-L. Zhu, hep-ph/0404123.

[91] S. Pakvasa and M. Suzuki, hep-ph/0402079.

[92] H. Harari and H. J. Lipkin, Phys. Rev. Lett. 13, 345 (1964).

[93] F. E. Low, Symmetries and Elementary Particles, (Gordon and Breach, New York, 1967).

[94] J. J. de Swart, Rev. Mod. Phys. 35, 916 (1963), 37, 326(E) (1965).

[95] F. E. Close, An Introduction to Quarks and Partons, (Academic Press, London, 1979). 
[96] Fayyazuddin and Riazuddin, A Modern Introduction to Particle Physics, (World Scientific, Singapore, 1992).

[97] Q. Zhao and F. E. Close, hep-ph/0404075.

[98] P. Carruthers and M. M. Nieto, Ann. Phys. (N.Y.) 51, 359 (1969).

[99] J. Haidenbauer and G. Krein, Phys. Rev. C 68, 052201 (2003).

[100] S. Nussinov, hep-ph/0307357; A. Casher and S. Nussinov, Phys. Lett. B 578, 124 (2004).

[101] R. A. Arndt, I. I. Strakovsky, and R. L. Workman, Phys. Rev. C 68, 042201 (2003), 69, 019901(E) (2004); nucl-th/0311030. 